\title{
Synthesis of an aminooxy derivative of the GM3 antigen and its application in oxime ligation.
}

Kristopher A. Kleski ${ }^{\dagger}$, Mengchao Shi ${ }^{\dagger}$, Matthew Lohman ${ }^{\dagger}$, Gabrielle T. Hymel ${ }^{\dagger}$, Vinod K. Gattoji ${ }^{\dagger}$, Peter R. Andreana*

$\dagger 2801$ West Bancroft Street, Department of Chemistry and Biochemistry and School of Green Chemistry and Engineering, The University of Toledo, Toledo, Ohio, 43606 United States.

*Correspondence and requests for materials should be addressed to P.R.A.

(email: peter.andreana@utoledo.edu)

\section{Supporting Information}

\section{Table of Contents}

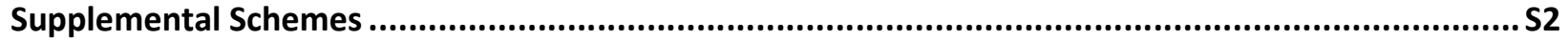

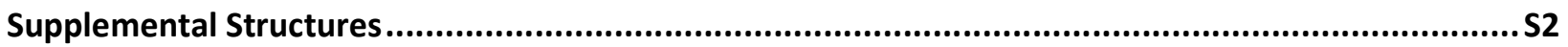

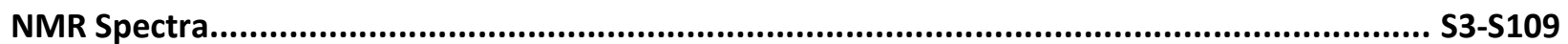




\section{Scheme S1}

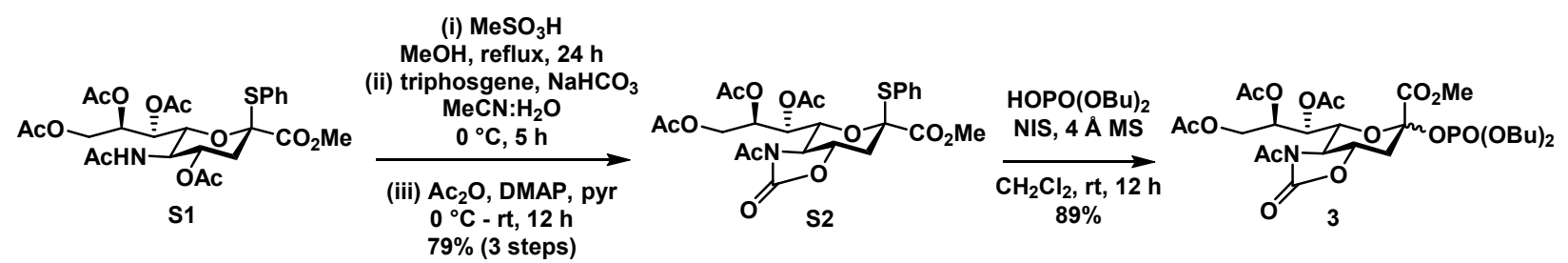

\section{Scheme S2}

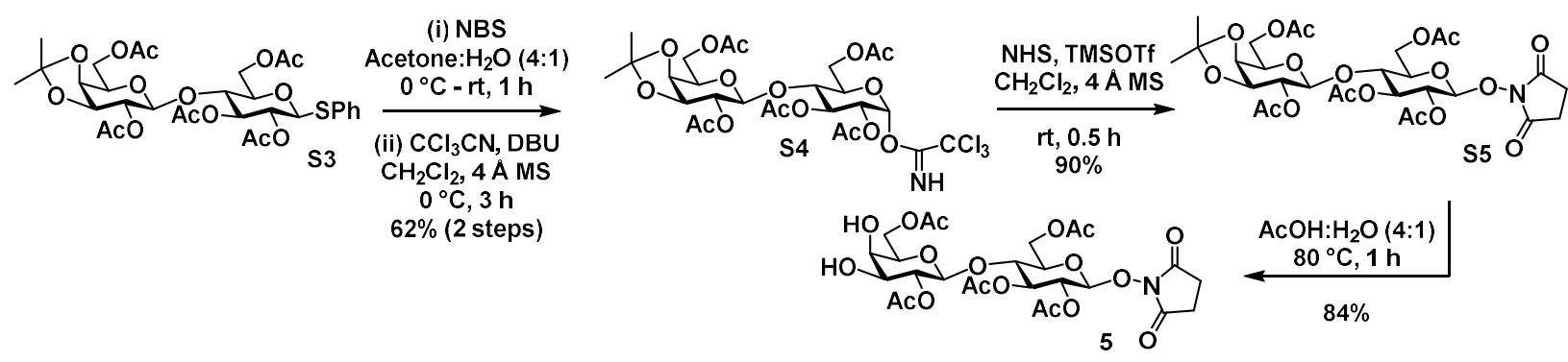

\section{Compound S6}

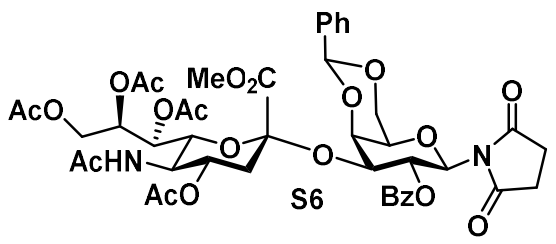

\section{Compound S7}

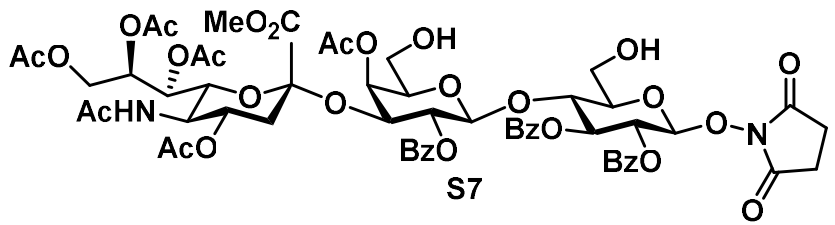




\section{$\underline{{ }^{1} \mathrm{H} N M R ; 600 \mathrm{MHz} ; \mathrm{CDCl}_{3}}$}

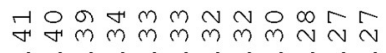

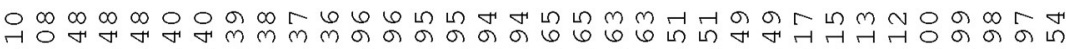

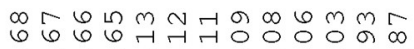

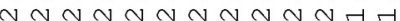

W

AcO $\underbrace{\mathrm{AcO} \text { OAc }}_{\substack{\mathrm{OAC} \\ \mathrm{S} 1}} \overbrace{\mathrm{CO}_{2} \mathrm{Me}}^{\mathrm{SPh}}$

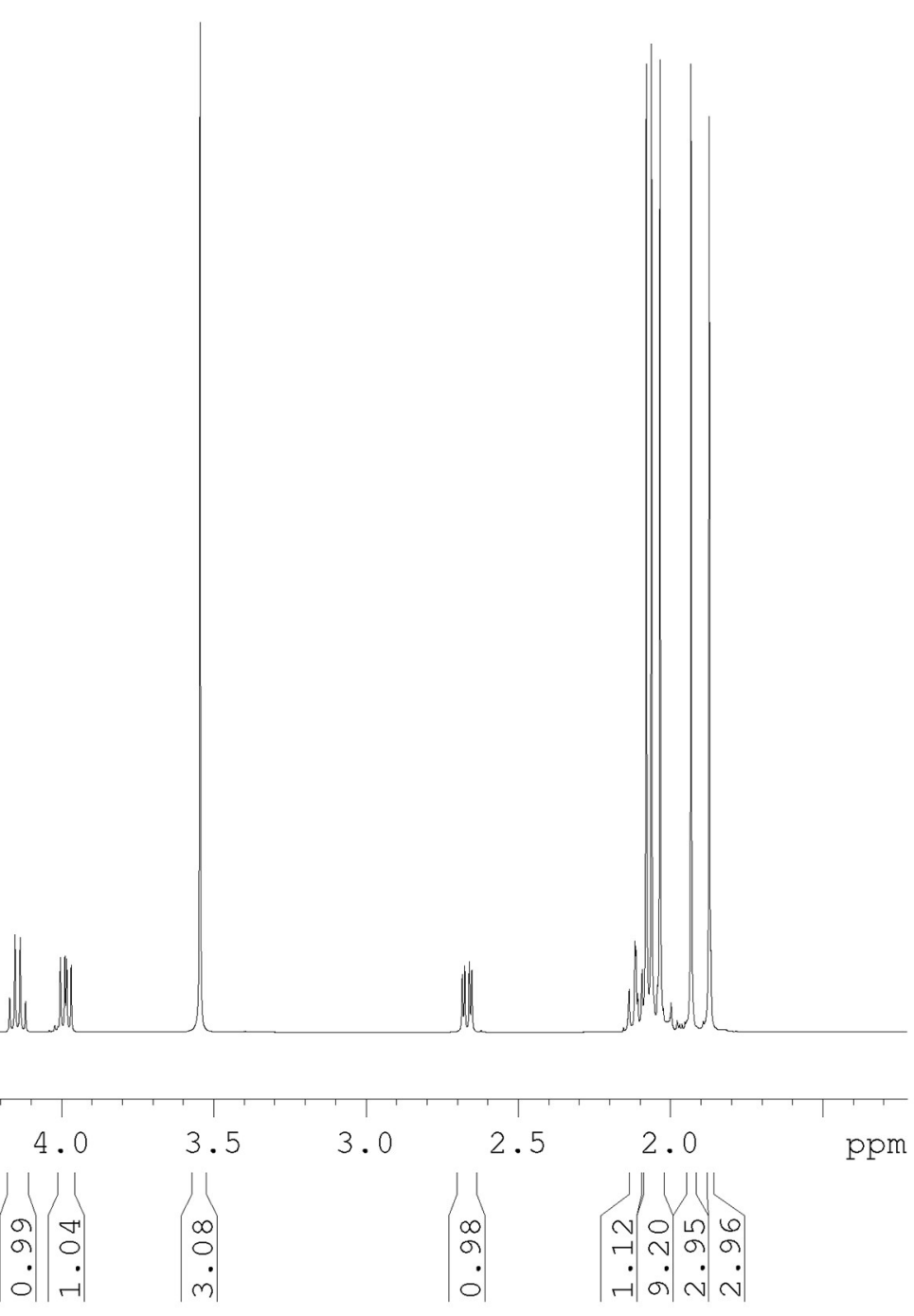




\section{${ }^{13} \mathrm{C} \mathrm{NMR} ; 150 \mathrm{MHz} ; \mathrm{CDCl}_{3}$}

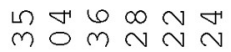
$\rightarrow$
$M$
மंकें
겅ㅇㅇㅇ
M $N$ N

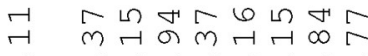
$\stackrel{8}{\mathrm{~N}}$

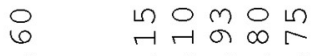
त्र

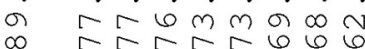
तु
mंत्ठ்.
$1<1<1$
n?
14

$\mathrm{AcO} \underbrace{\mathrm{SP}}_{\mathrm{AcHN}-\underbrace{\mathrm{AcO}}_{\mathrm{OAC}} \mathrm{OAC}_{\mathrm{S} 1}^{\mathrm{SPh}}} \mathrm{CO}_{2} \mathrm{Me}$
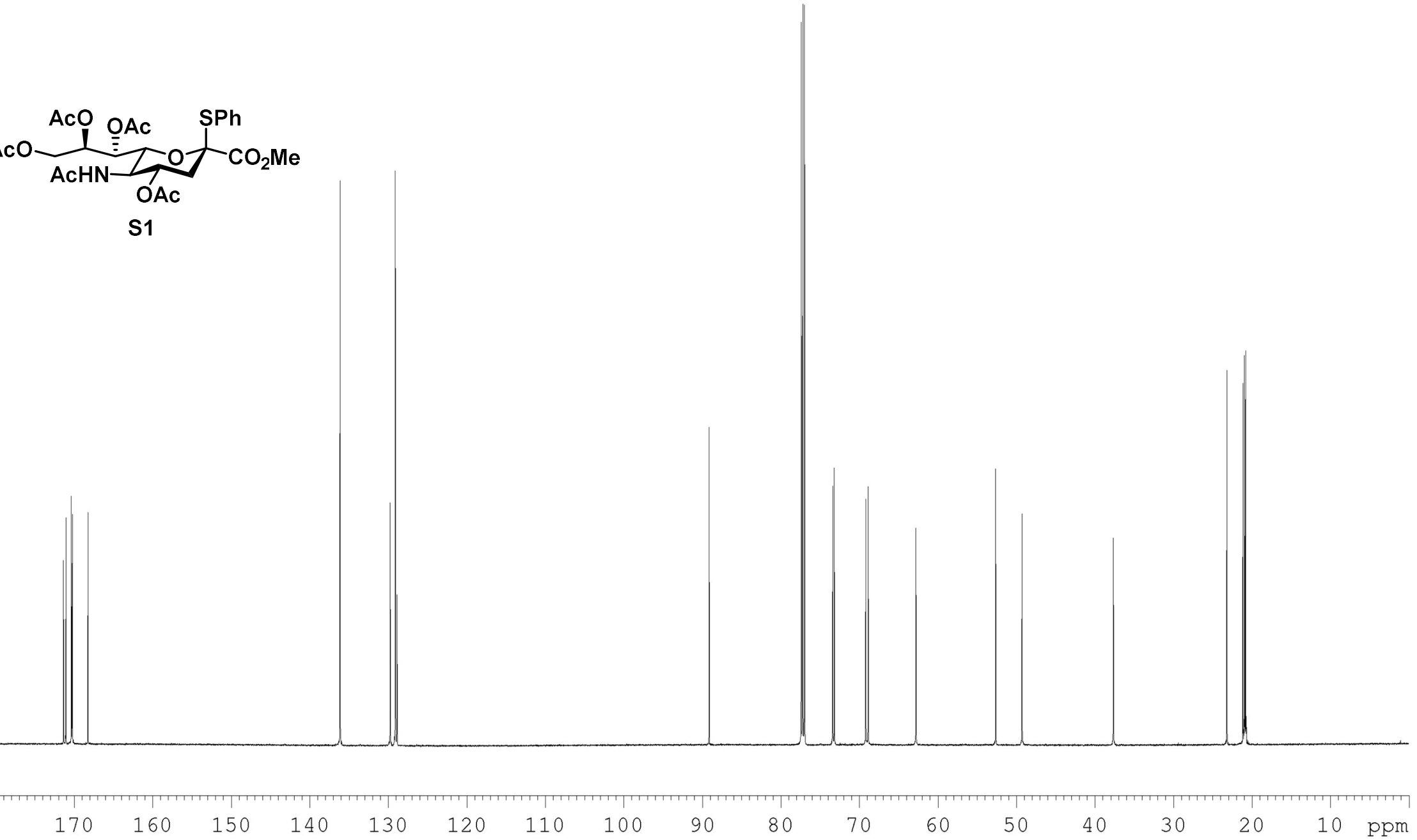


\section{$\underline{{ }^{1} \mathrm{H} N M R ; 600 \mathrm{MHz} ; \mathrm{CDCl}_{3}}$}

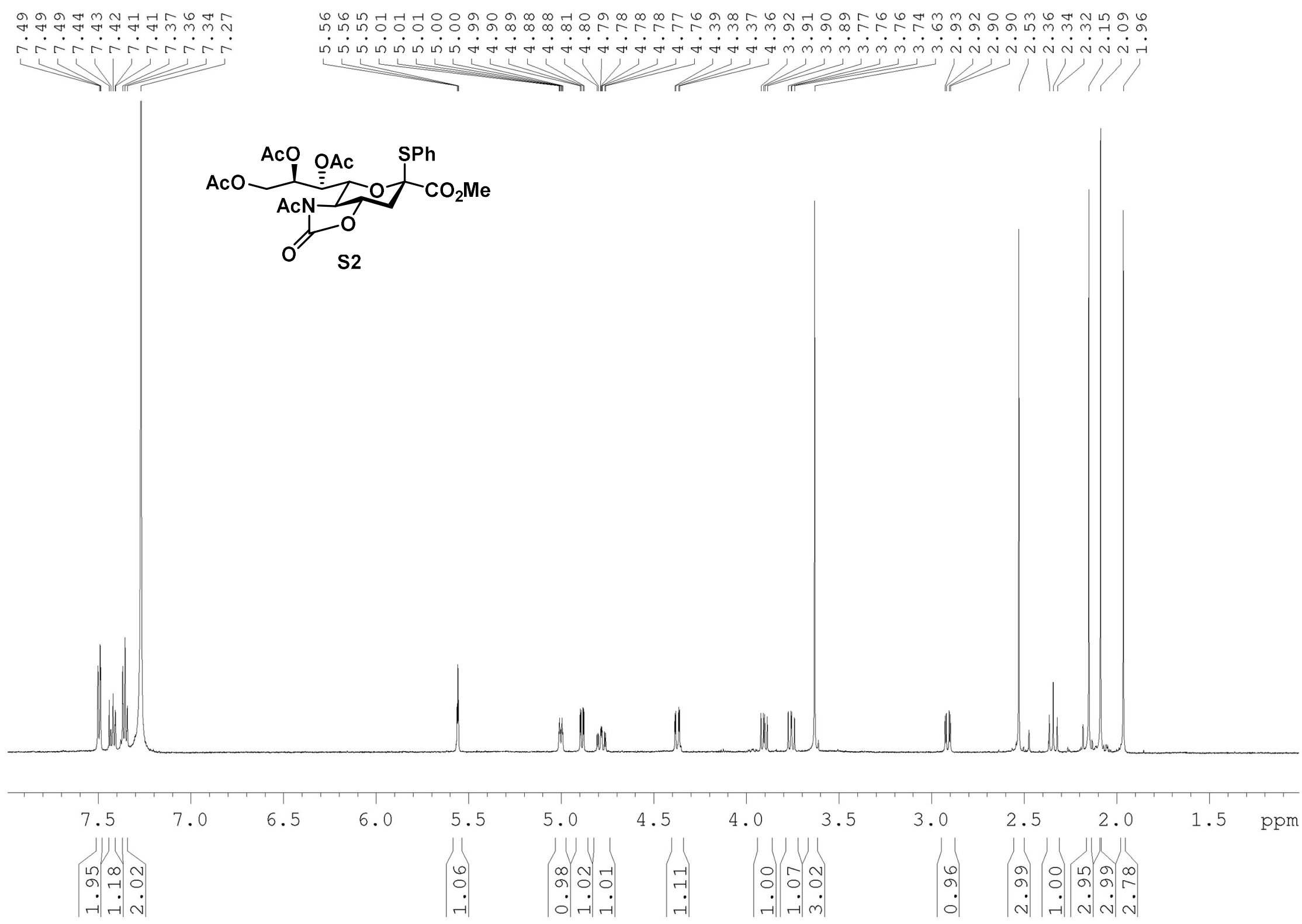


$\underline{{ }^{13} \mathrm{C} \mathrm{NMR} ; 150 \mathrm{MHz} ; \mathrm{CDCl}_{3}}$

\begin{tabular}{|c|c|c|c|}
\hline$N G+\sigma$ & $\infty$ & & \\
\hline mar & เ & r & \\
\hline$N \not \circ \sigma \hat{\sigma}$ & $m$ & 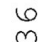 & \\
\hline 닥닥 & -1 & -1 & \\
\hline
\end{tabular}

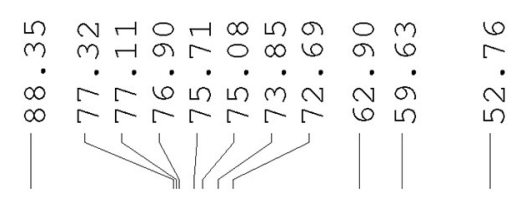

0 m

m $\quad \dot{\pi} \stackrel{\text { में }}{\dot{N}}$

然
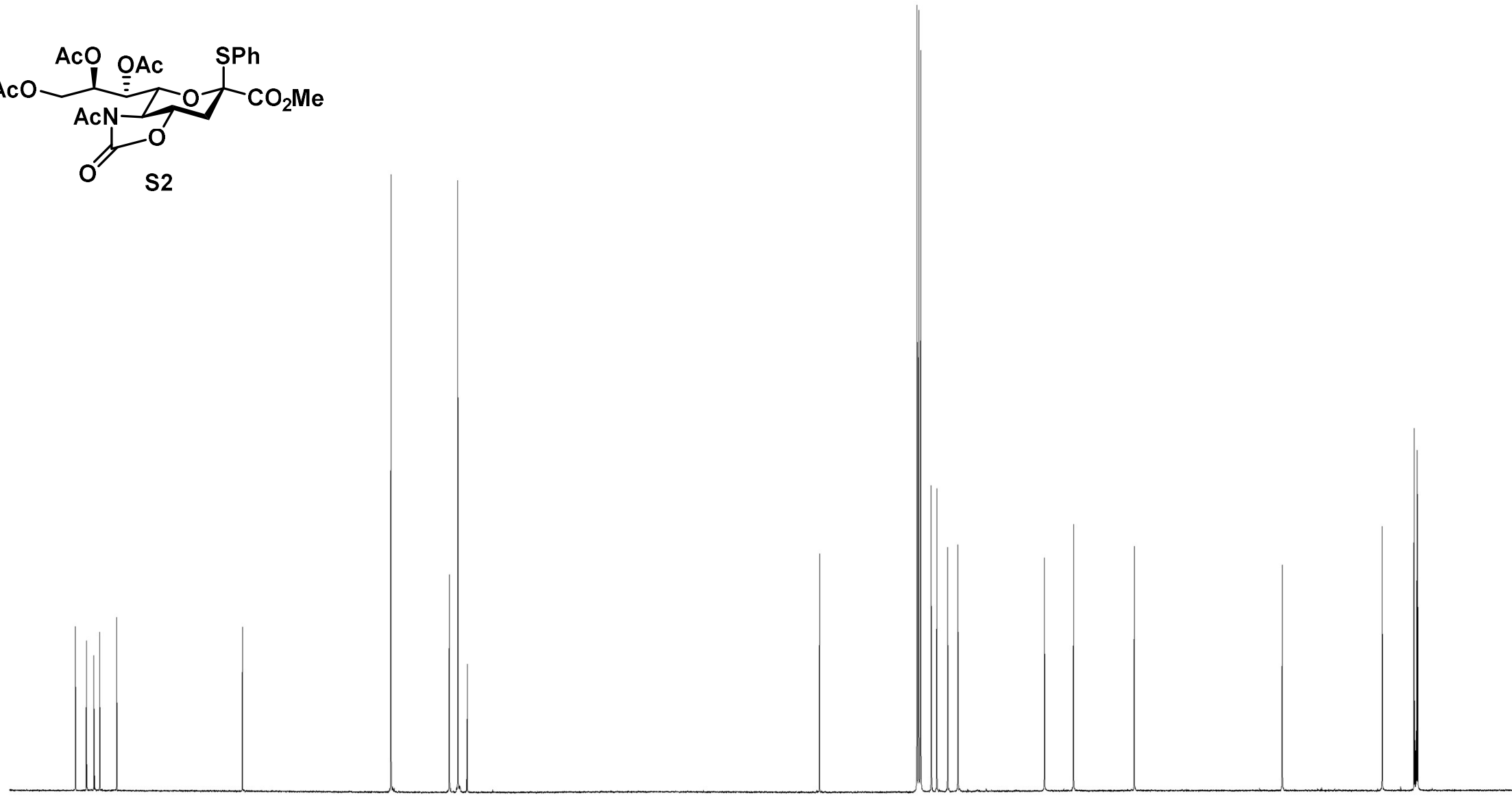

50

140

130

120

110

00

90

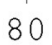

70

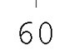

50

40 


\section{${ }^{1} \mathrm{H}$ NMR; $600 \mathrm{MHz} ; \mathrm{CDCl}_{3}$}

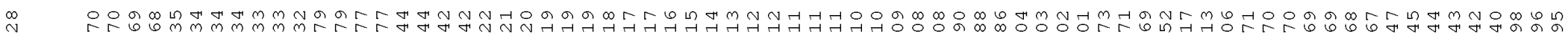

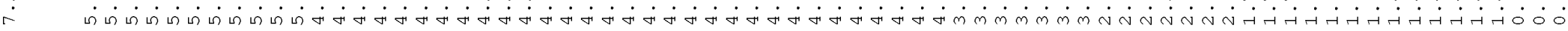
$\underbrace{\mathrm{N}}_{\mathrm{T}}$

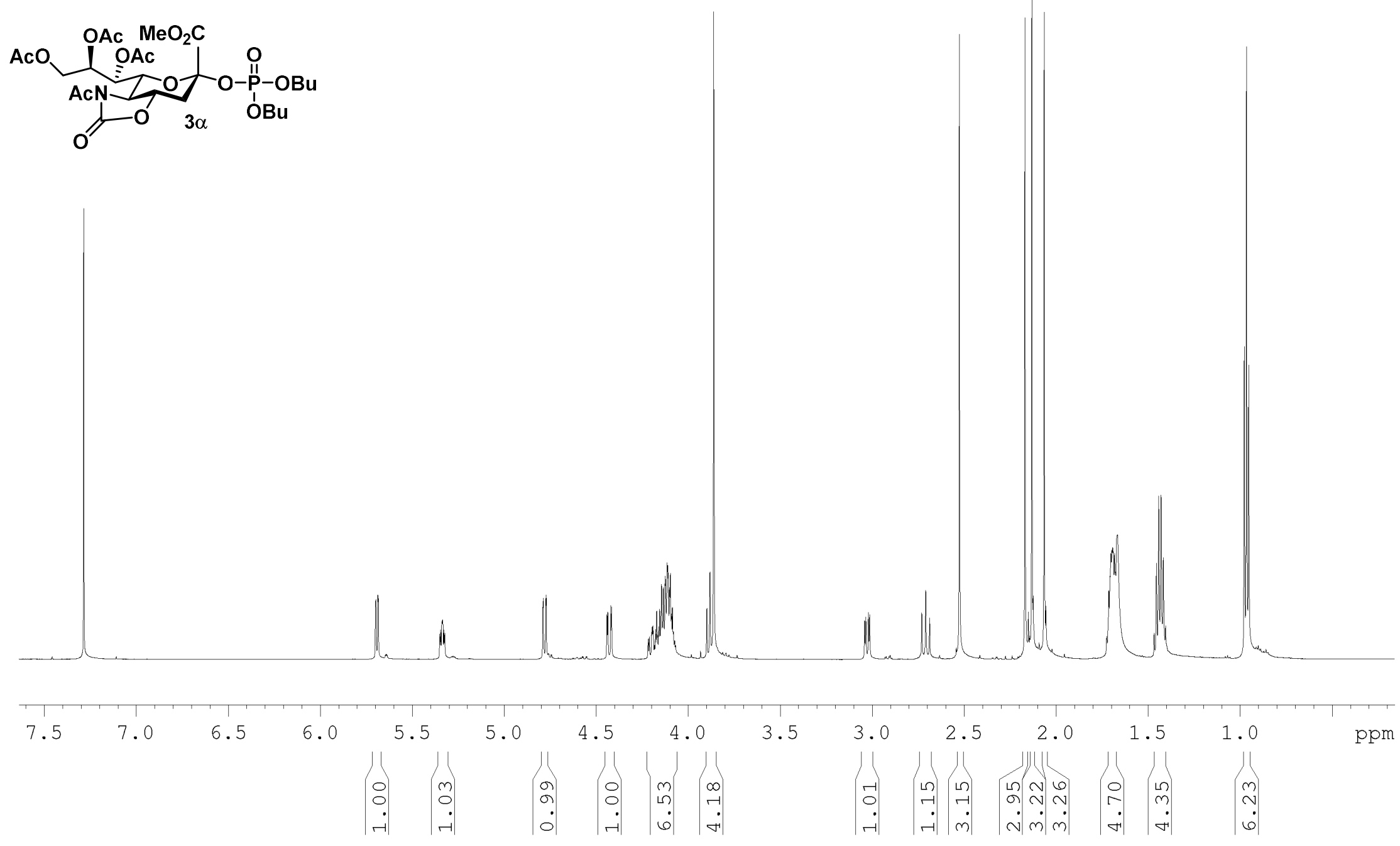


${ }^{13} \mathrm{C} \mathrm{NMR} ; 150 \mathrm{MHz} ; \mathrm{CDCl}_{3}$

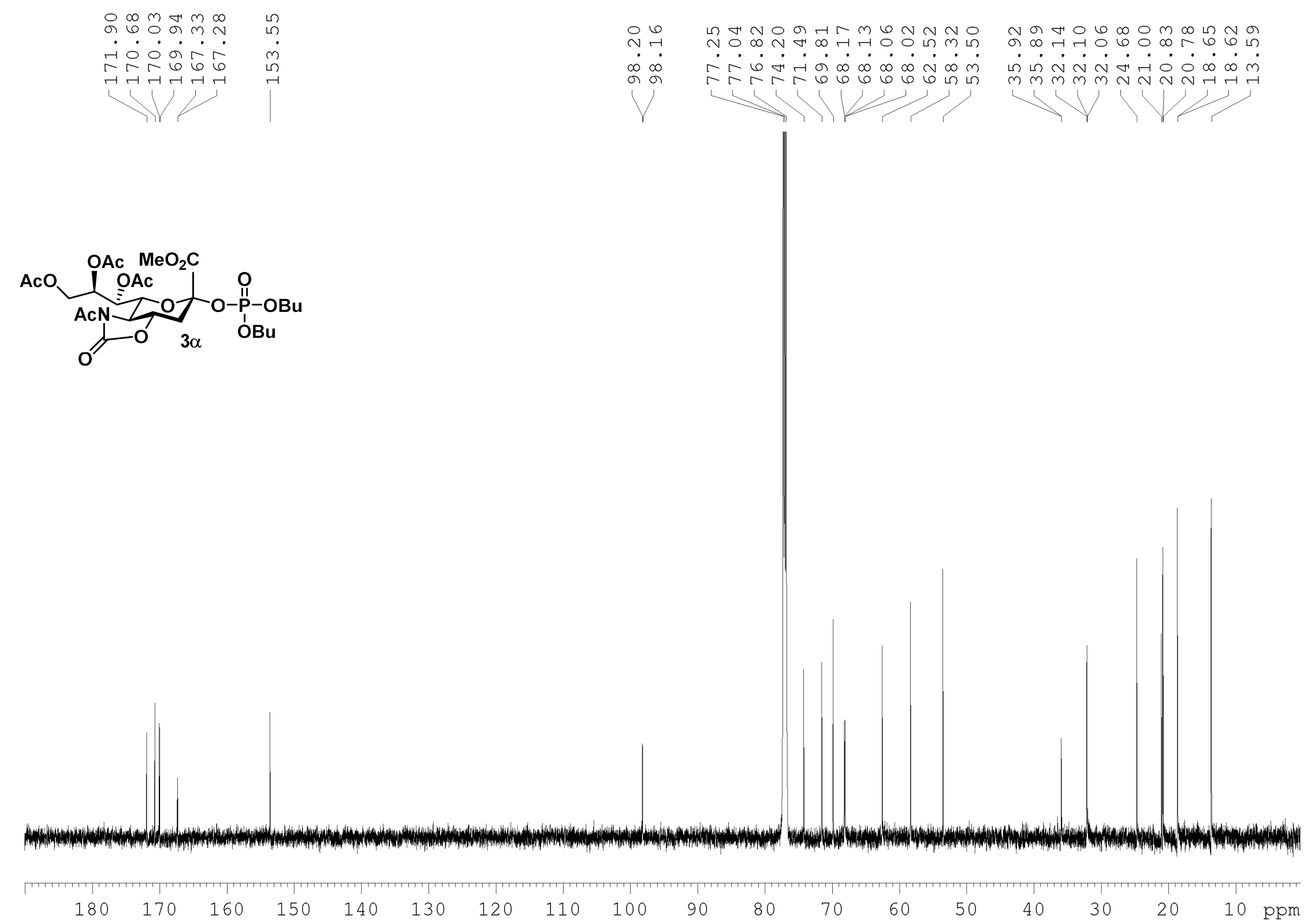




\section{$\underline{\text { COSY NMR; } 600 \mathrm{MHz} ; \mathrm{CDCl}_{3}}$}

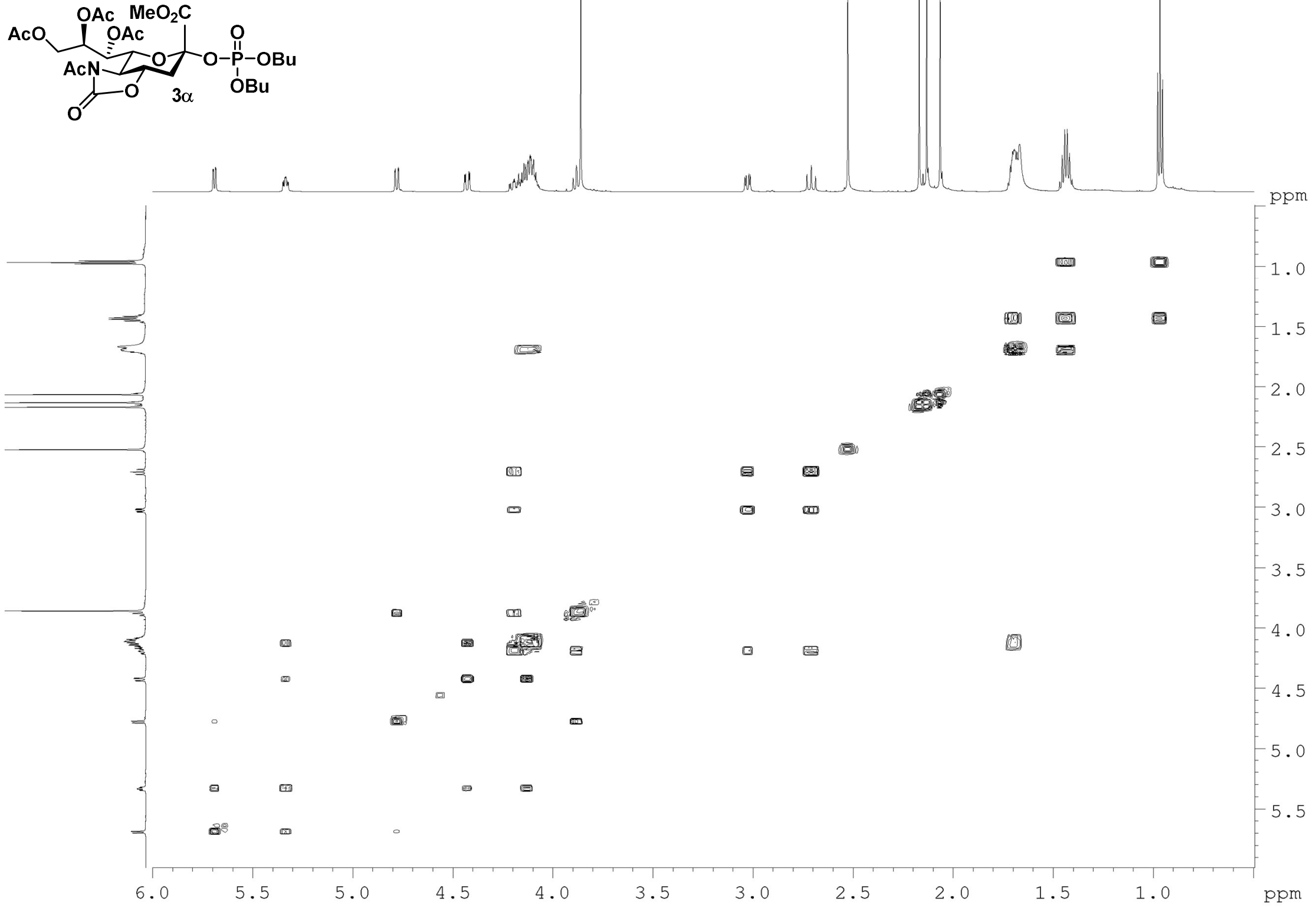


${ }^{1} \mathrm{H}$ NMR; $600 \mathrm{MHz} ; \mathrm{CDCl}_{3}$

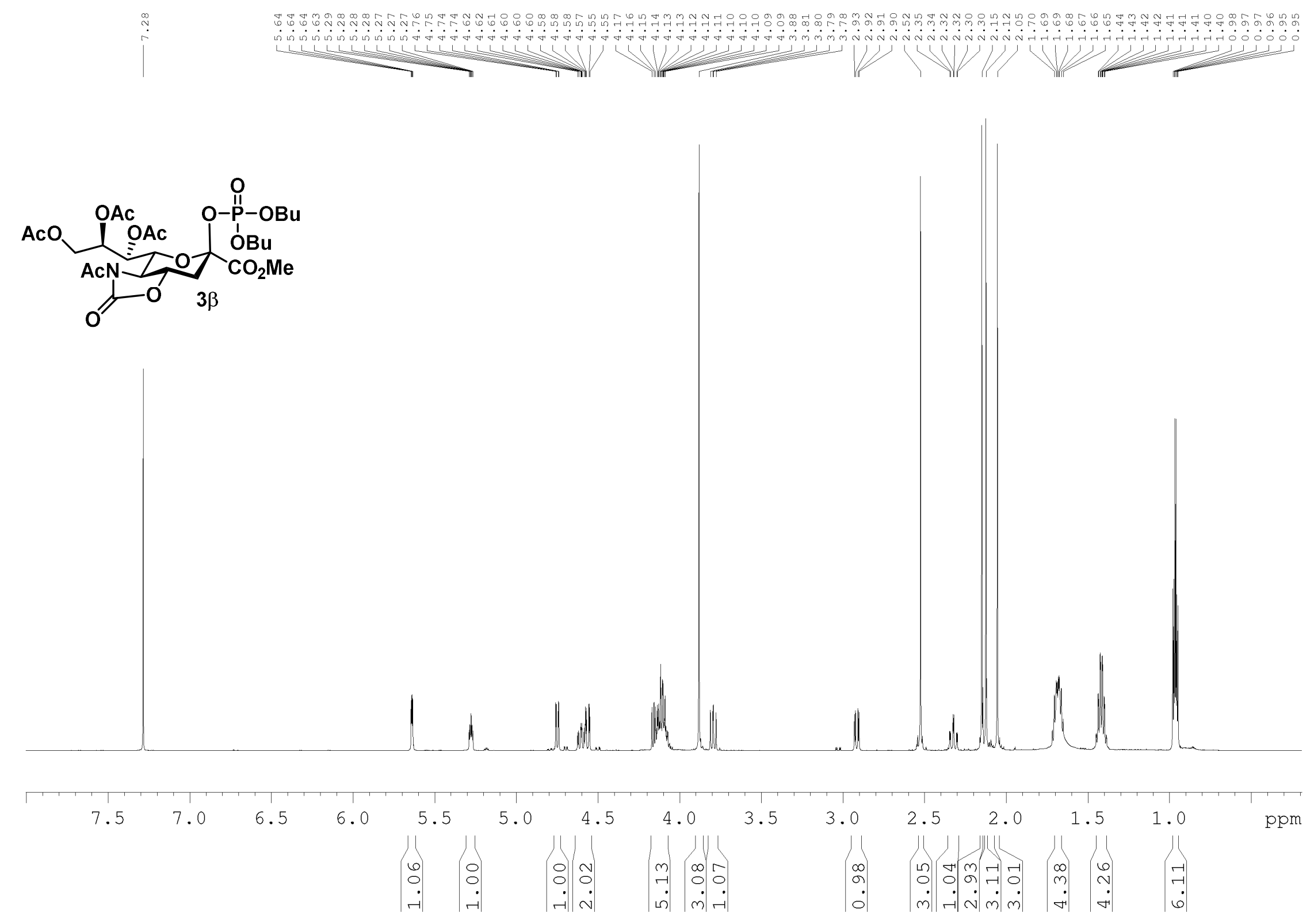


${ }^{13} \mathrm{C} \mathrm{NMR} ; 150 \mathrm{MHz} ; \mathrm{CDCl}_{3}$

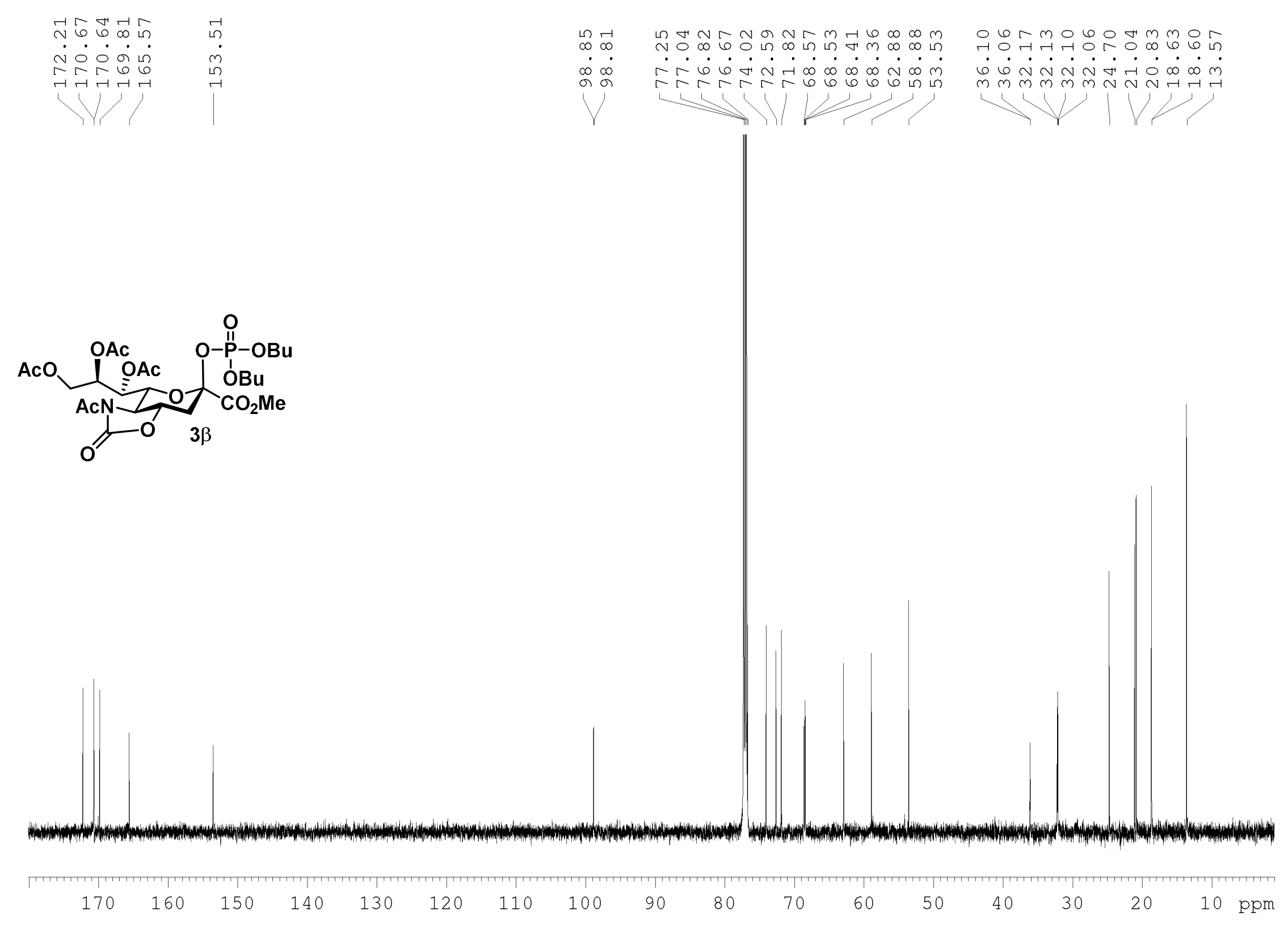


COSY NMR; $600 \mathrm{MHz} ; \mathrm{CDCl}_{3}$

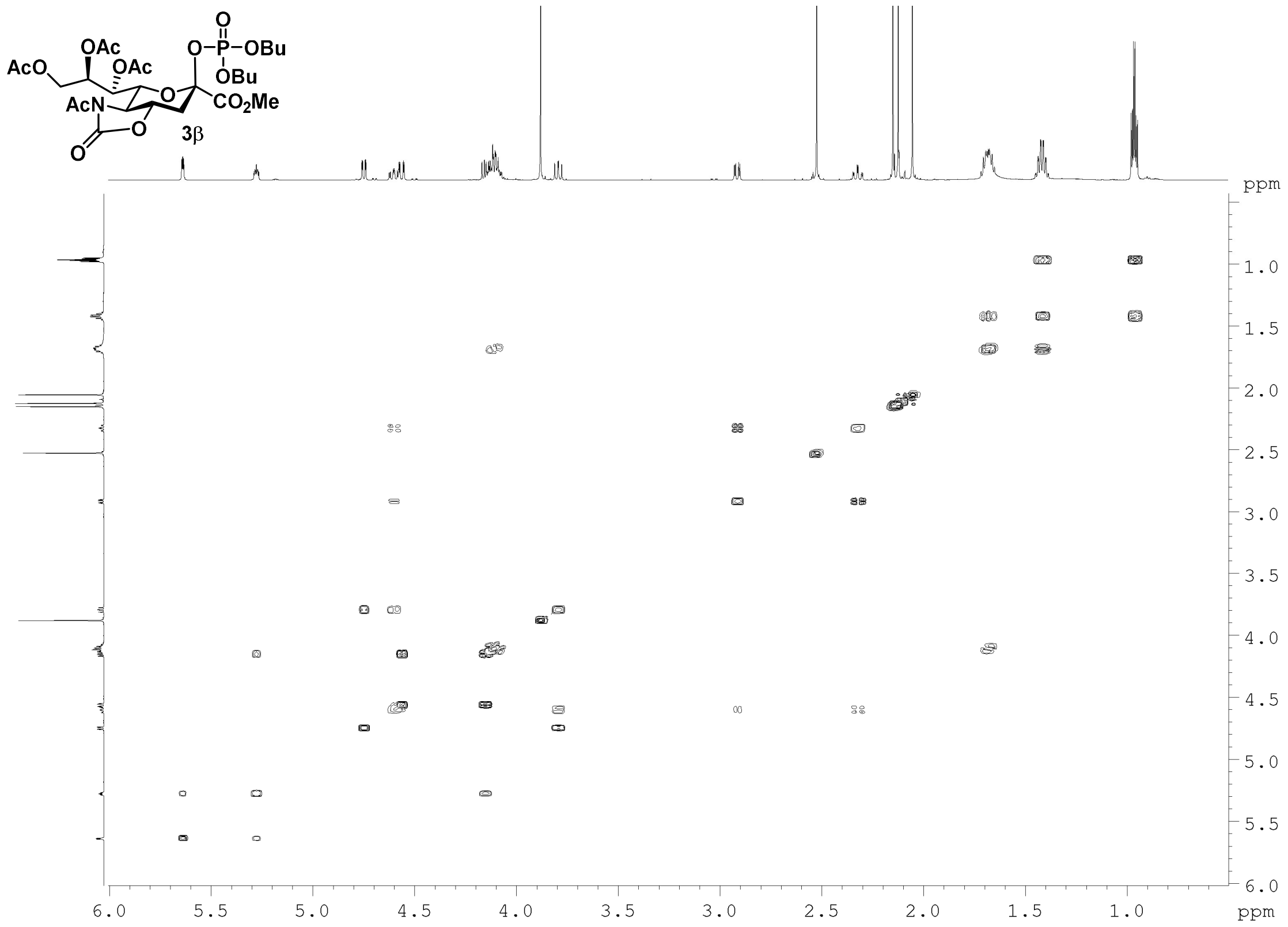




\section{${ }^{1} \mathrm{H} \mathrm{NMR} ; 600 \mathrm{MHz} ; \mathrm{CDCl}_{3}$}

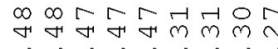

ririsirisit

$+$

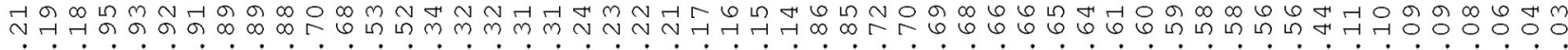

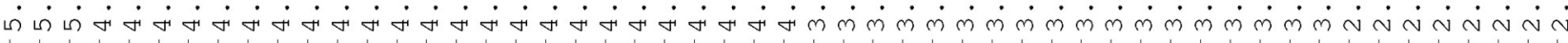
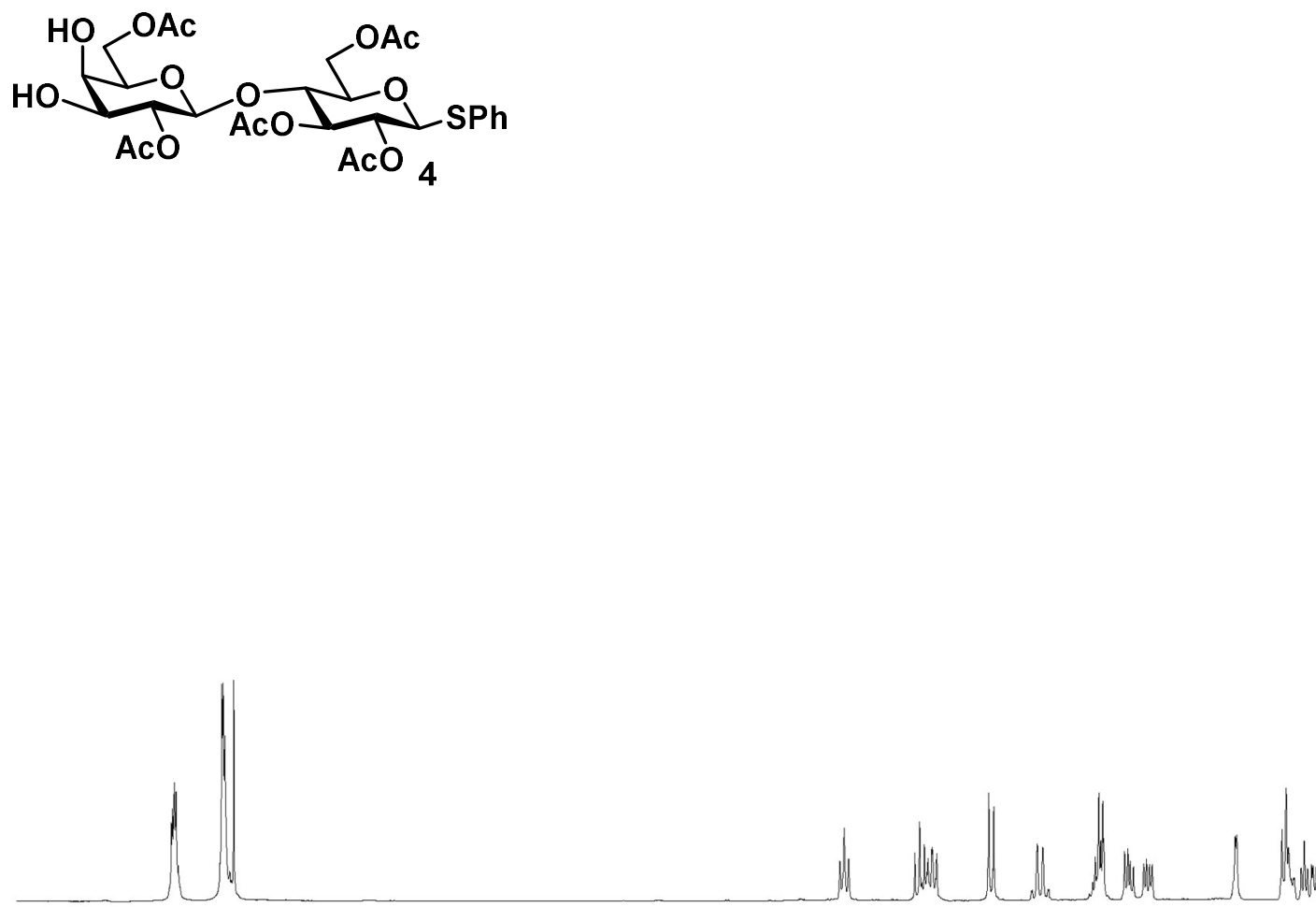

乎

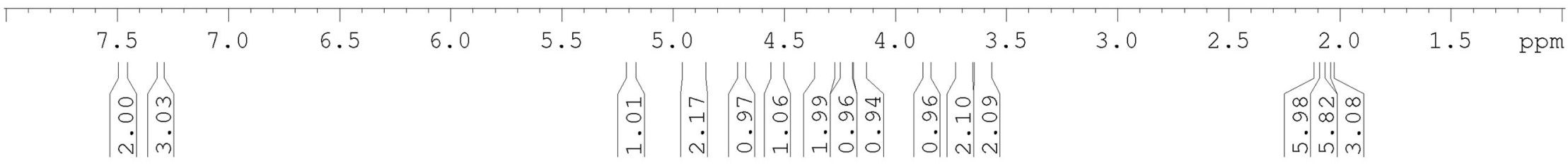


${ }^{13} \mathrm{C} \mathrm{NMR} ; 150 \mathrm{MHz} ; \mathrm{CDCl}_{3}$
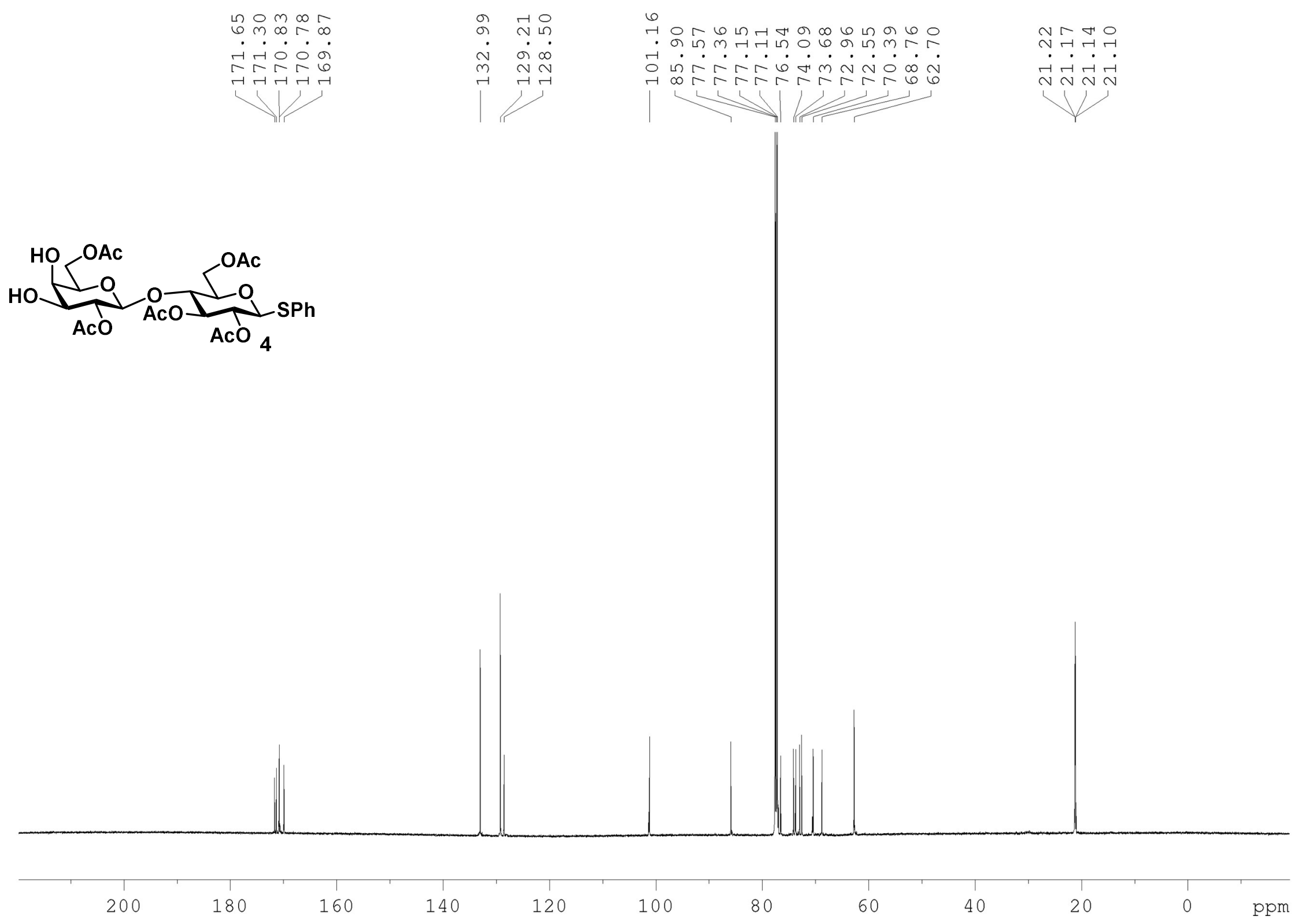


\section{${ }^{1} \mathrm{H} \mathrm{NMR} ; 600 \mathrm{MHz} ; \mathrm{CDCl}_{3}$}

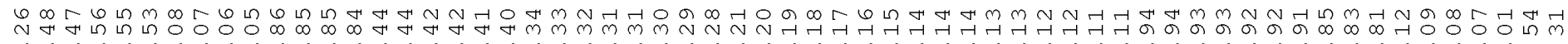

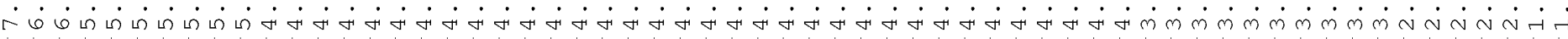

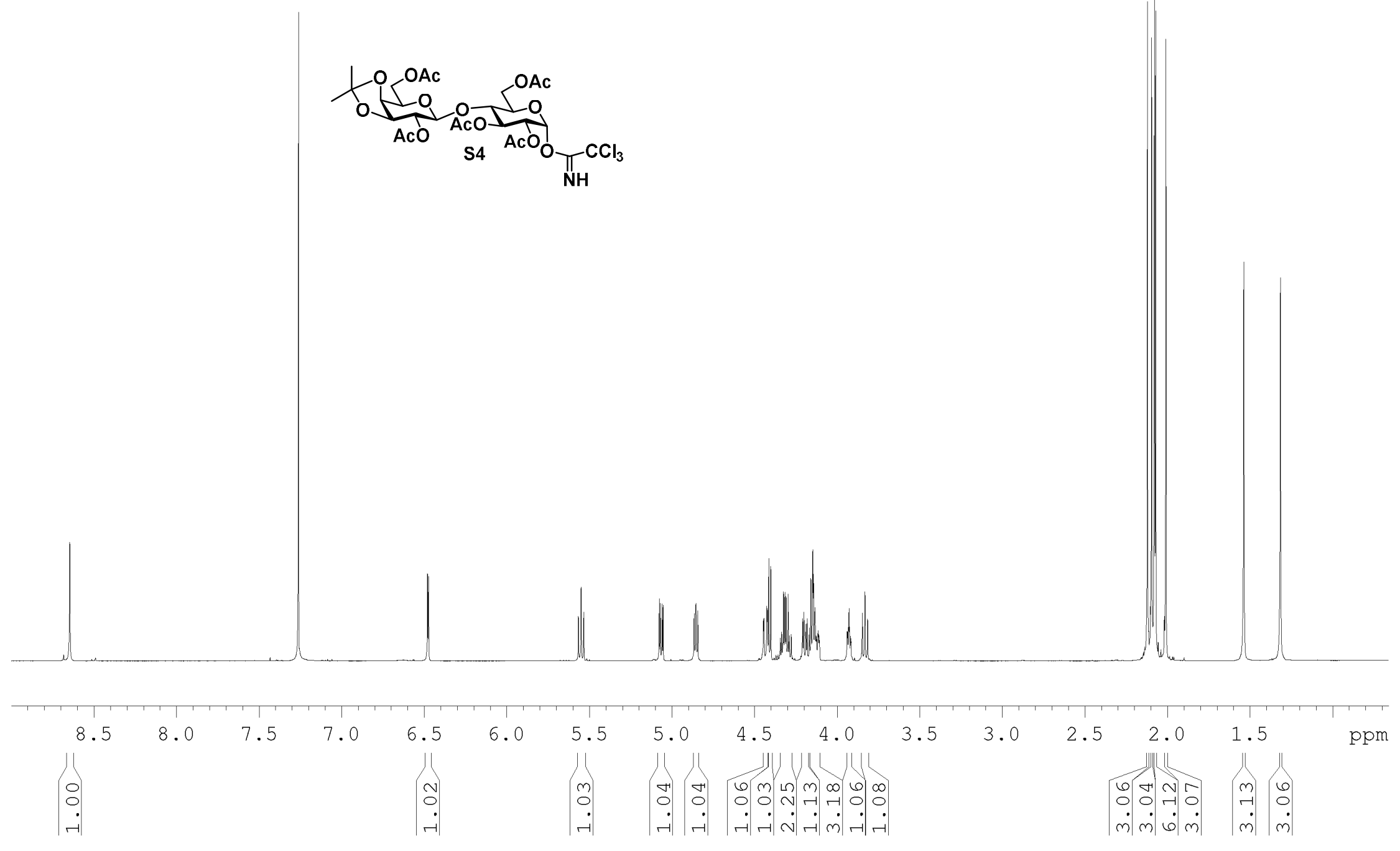


$\underline{{ }^{13} \mathrm{C} \mathrm{NMR} ; 150 \mathrm{MHz} ; \mathrm{CDCl}_{3}}$

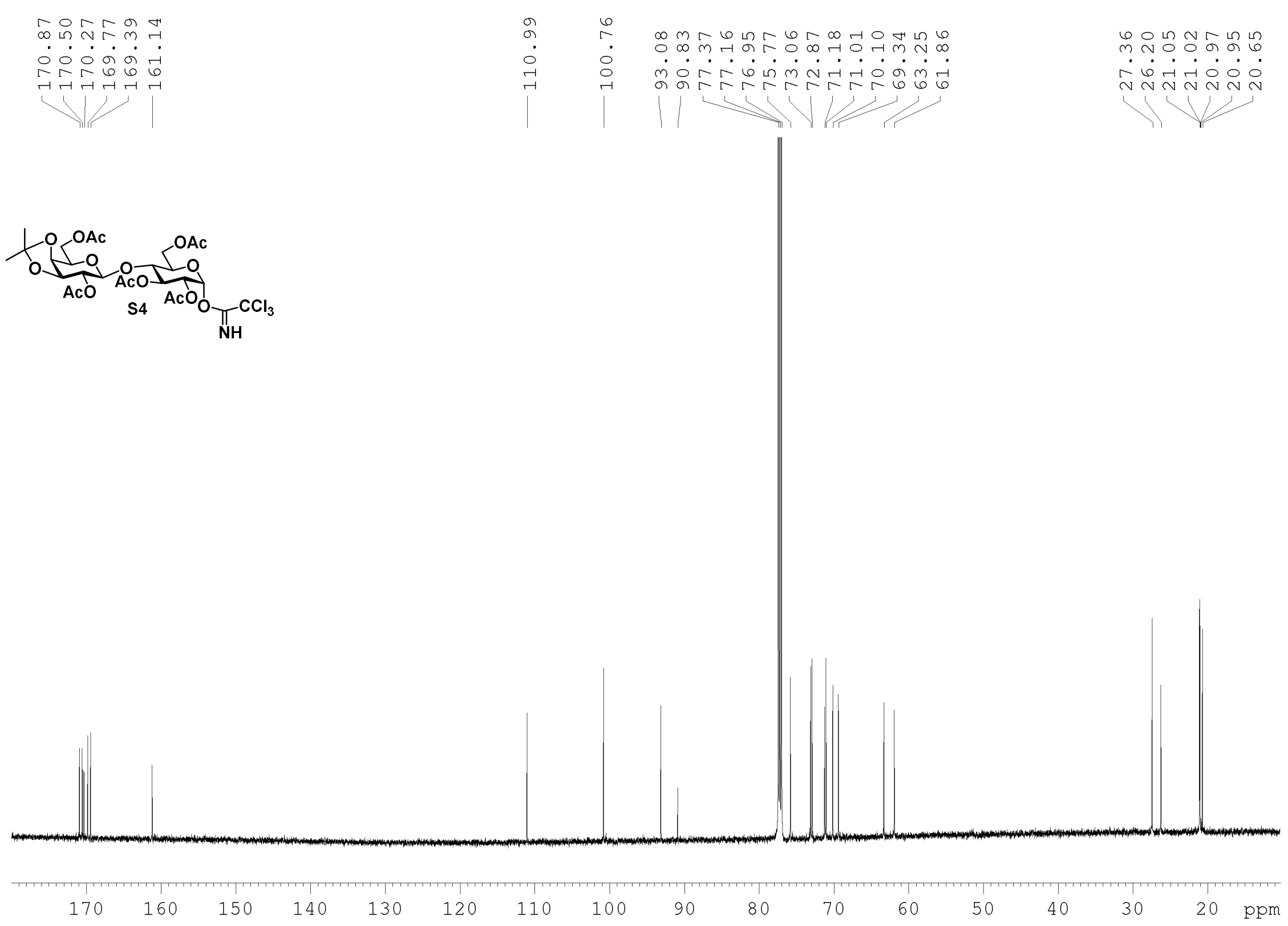




\section{${ }^{1} \mathrm{H}$ NMR; $600 \mathrm{MHz} ; \mathrm{CDCl}_{3}$}

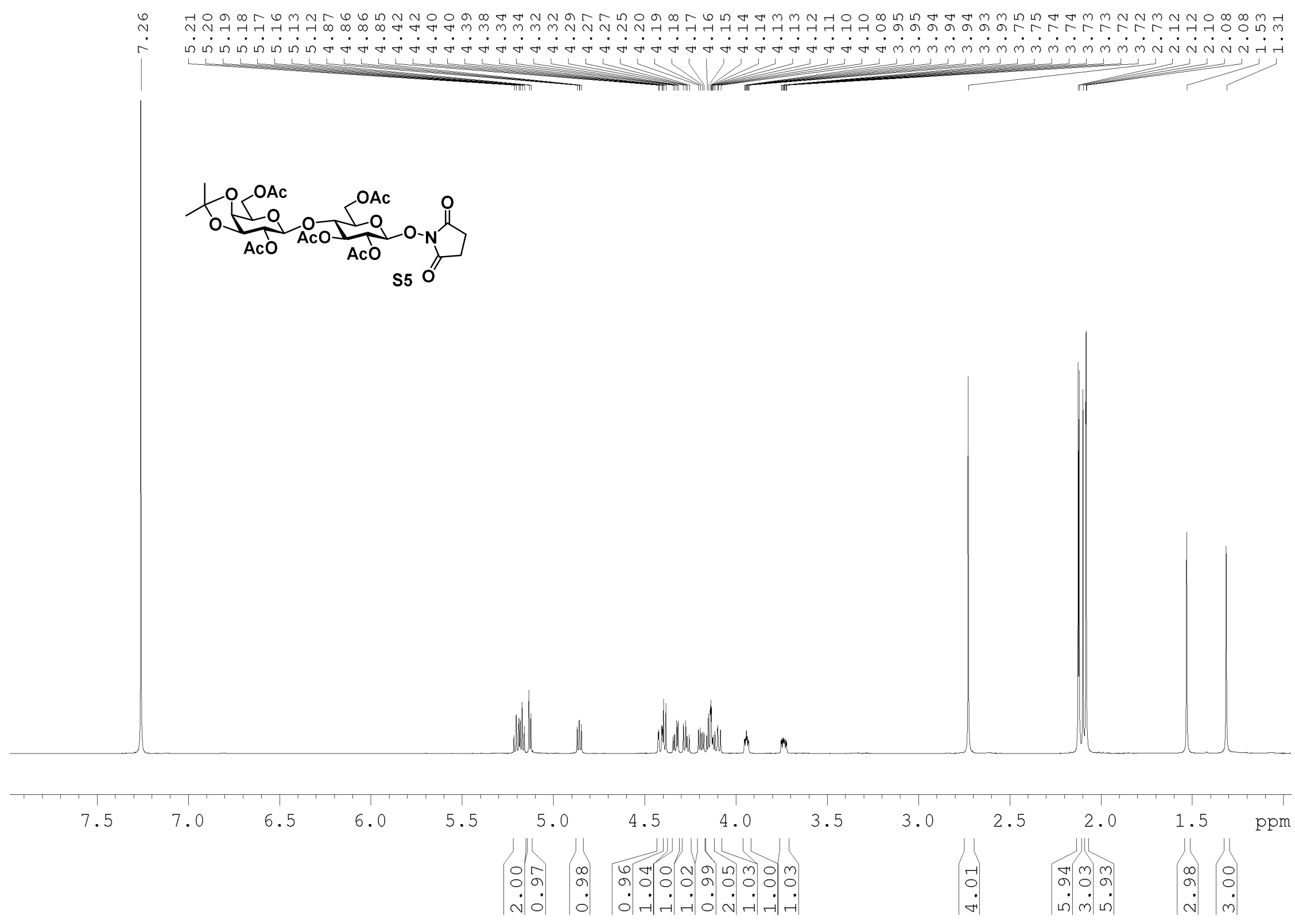




\section{${ }^{13} \mathrm{C} \mathrm{NMR} ; 150 \mathrm{MHz} ; \mathrm{CDCl}_{3}$}

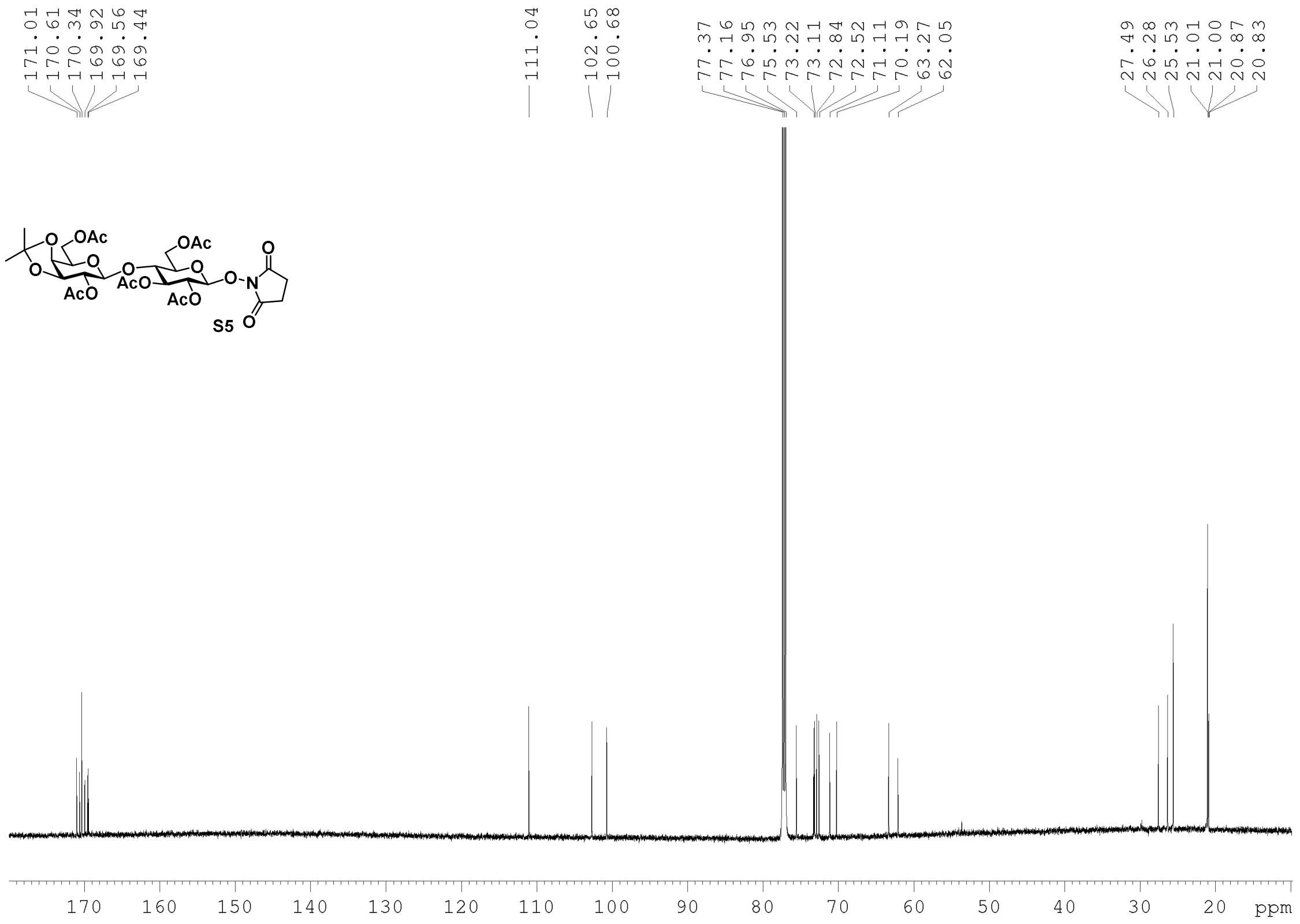




\section{${ }^{1} \mathrm{H} \mathrm{NMR} ; 600 \mathrm{MHz} ; \mathrm{CDCl}_{3}$}

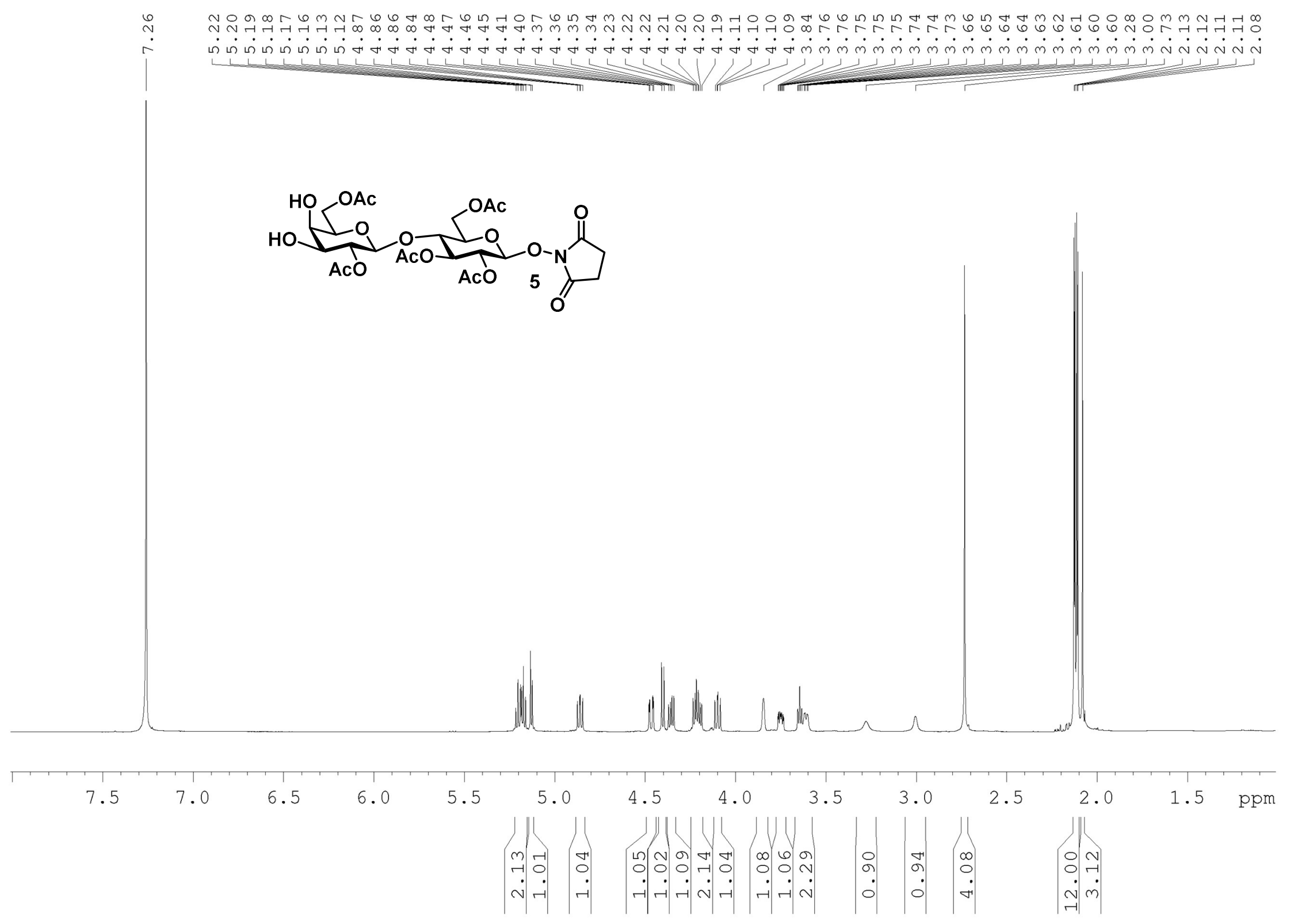


$\underline{{ }^{13} \mathrm{C} \mathrm{NMR} ; 150 \mathrm{MHz} ; \mathrm{CDCl}_{3}}$

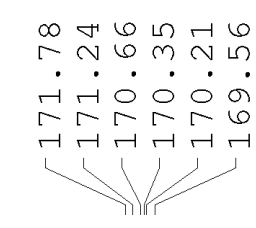

ॠथ

$\dot{\sim} \dot{\circ}$

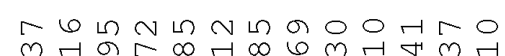

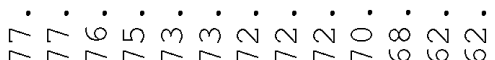

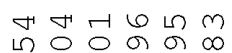

मेंत्रें0

प।

(1)

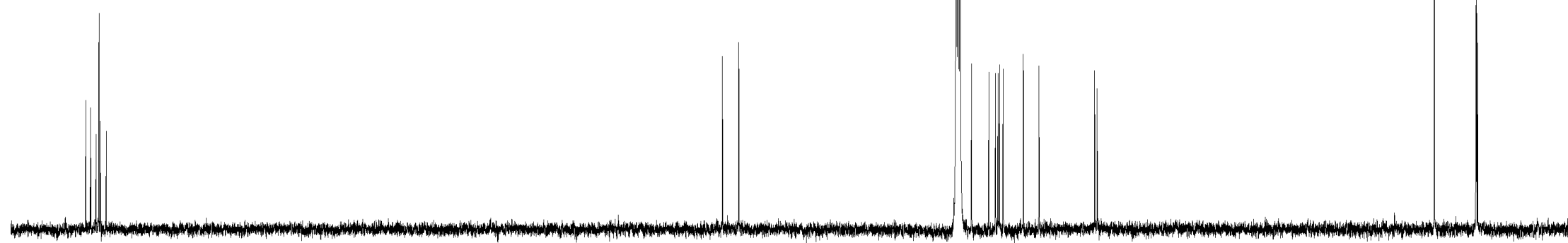

17

$160 \quad 150$

140

130

120

110

100

90

80

70

60

50

40

30

20 ppm 
${ }^{1} \mathrm{H}$ NMR; $600 \mathrm{MHz} ; \mathrm{CDCl}_{3}$

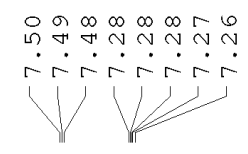

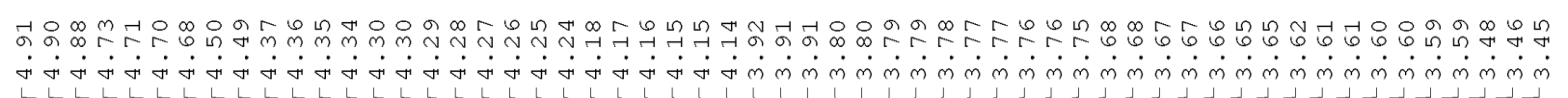
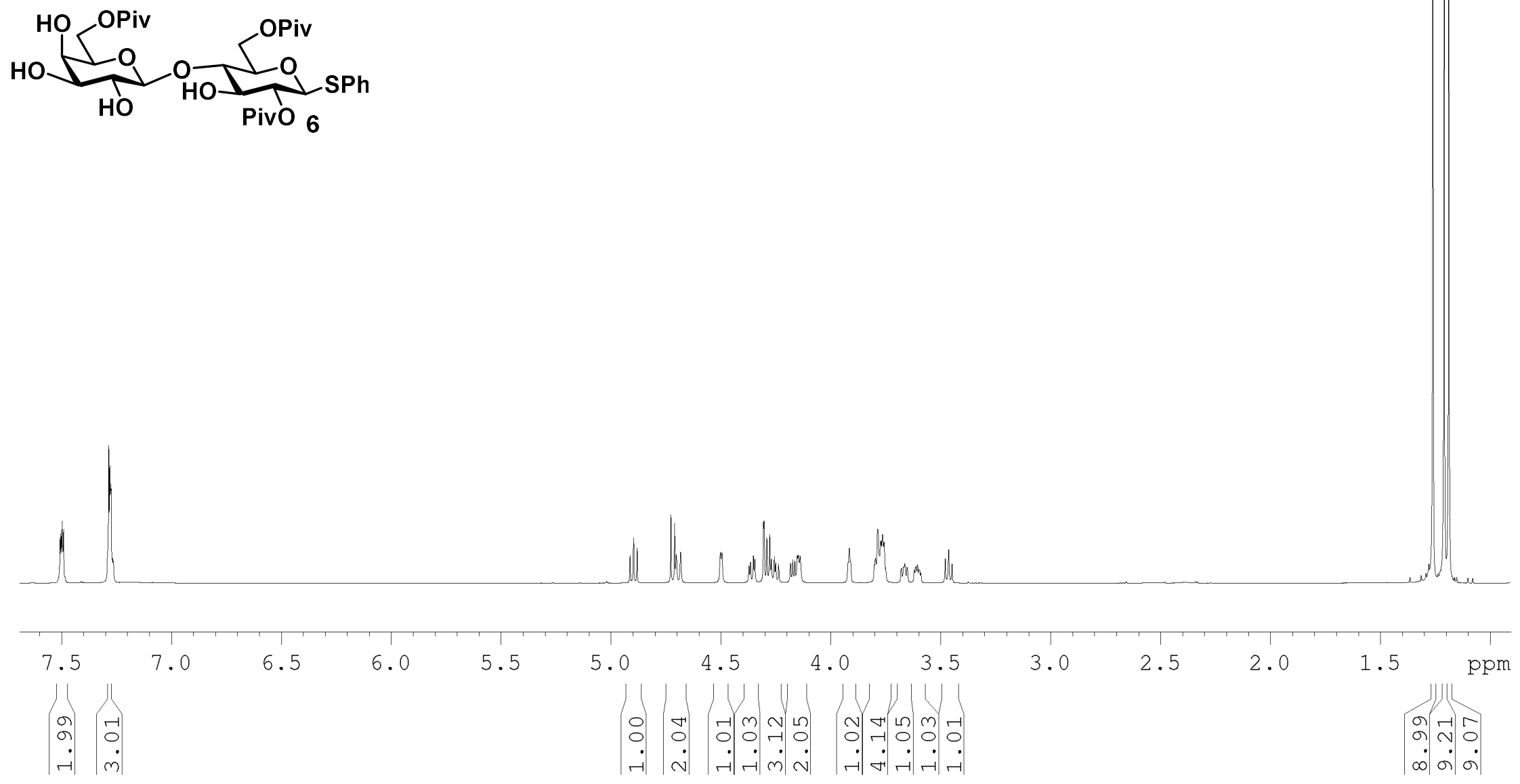

S21 
${ }^{13} \mathrm{C} \mathrm{NMR} ; 150 \mathrm{MHz} ; \mathrm{CDCl}_{3}$

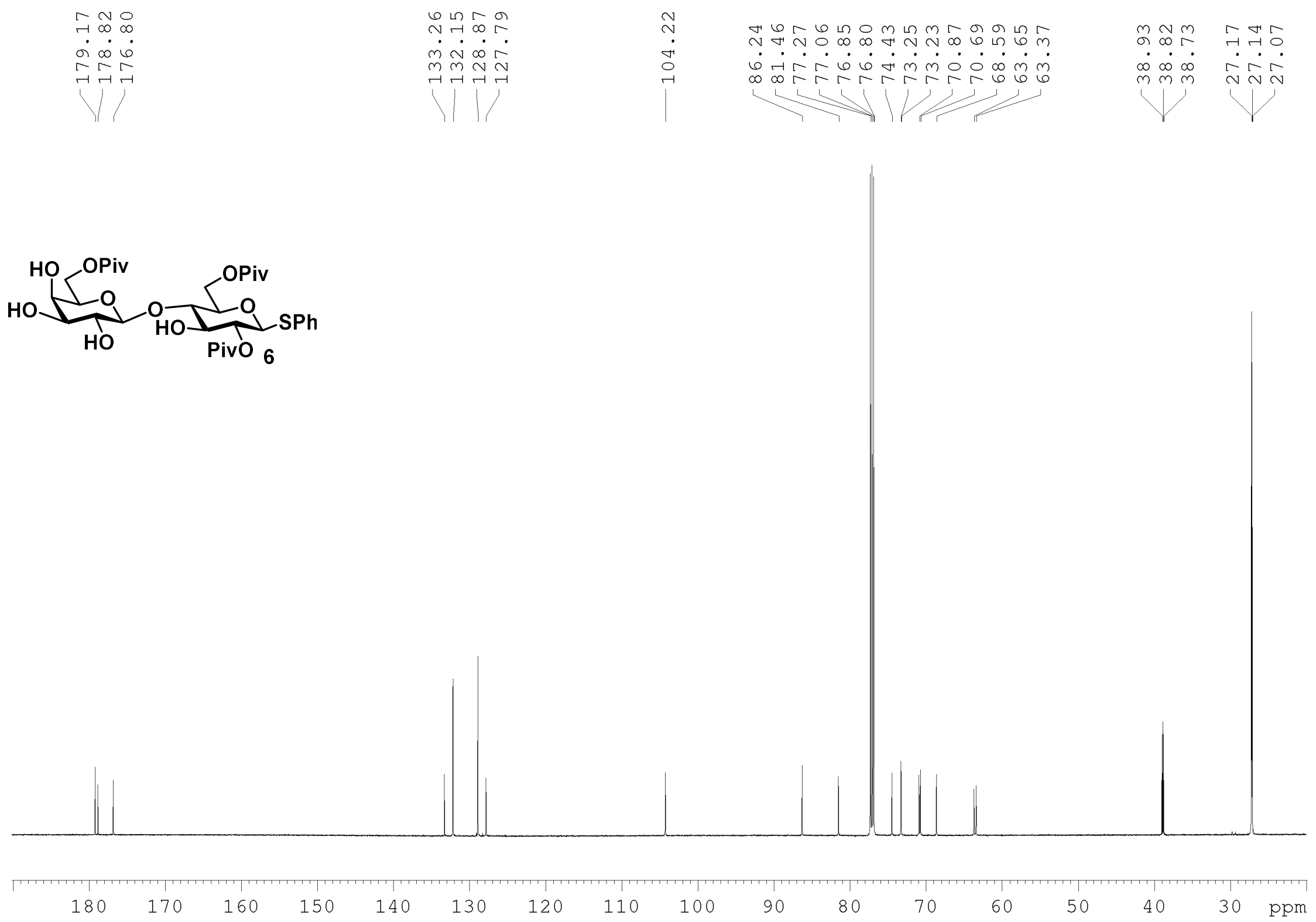




\section{${ }^{1} \mathrm{H}$ NMR; $600 \mathrm{MHz} ; \mathrm{CDCl}_{3}$}

국해

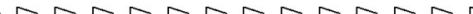

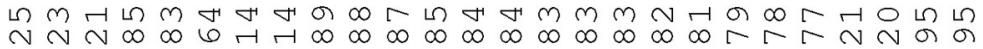

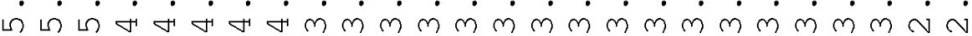
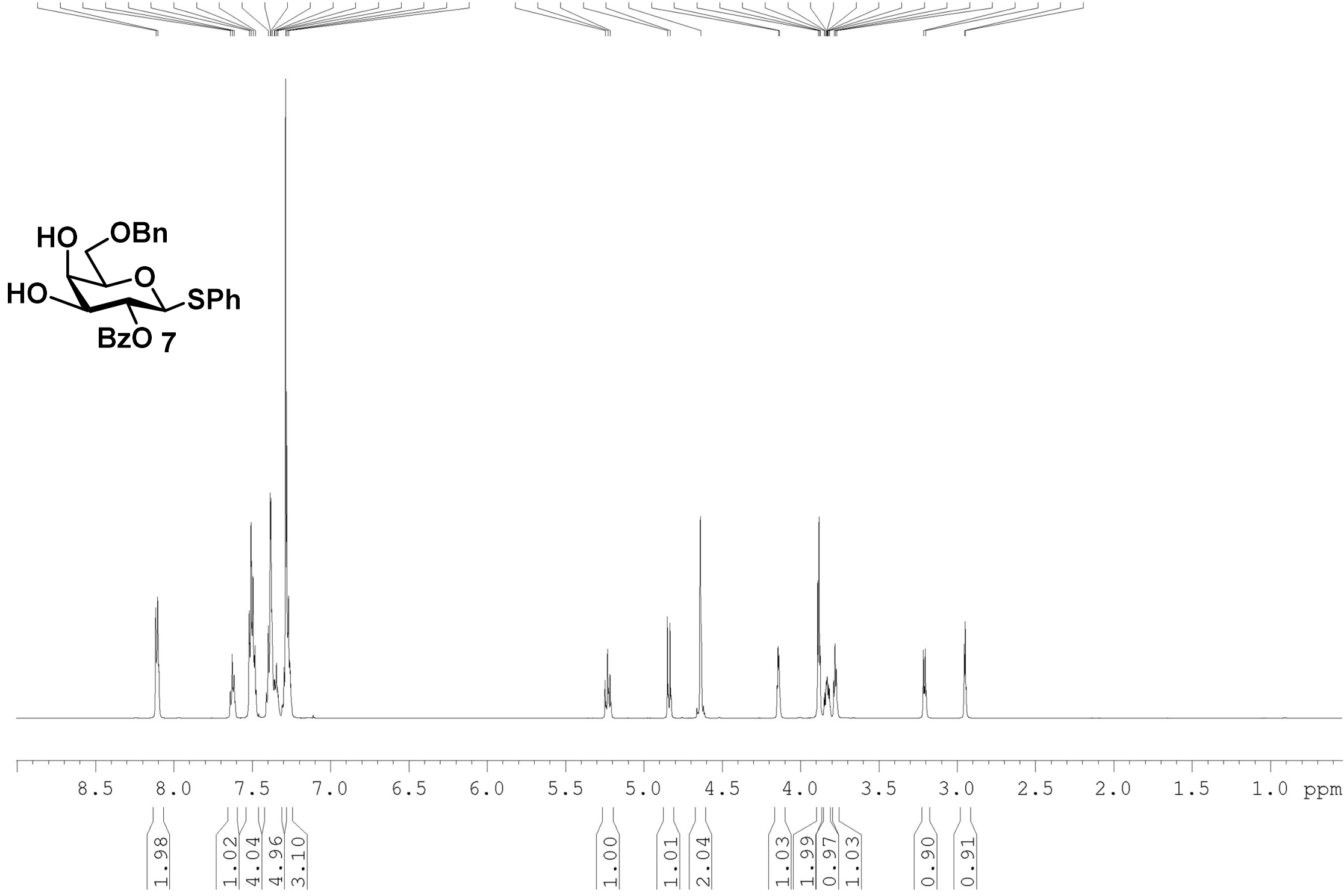
${ }^{13} \mathrm{C} \mathrm{NMR} ; 150 \mathrm{MHz} ; \mathrm{CDCl}_{3}$
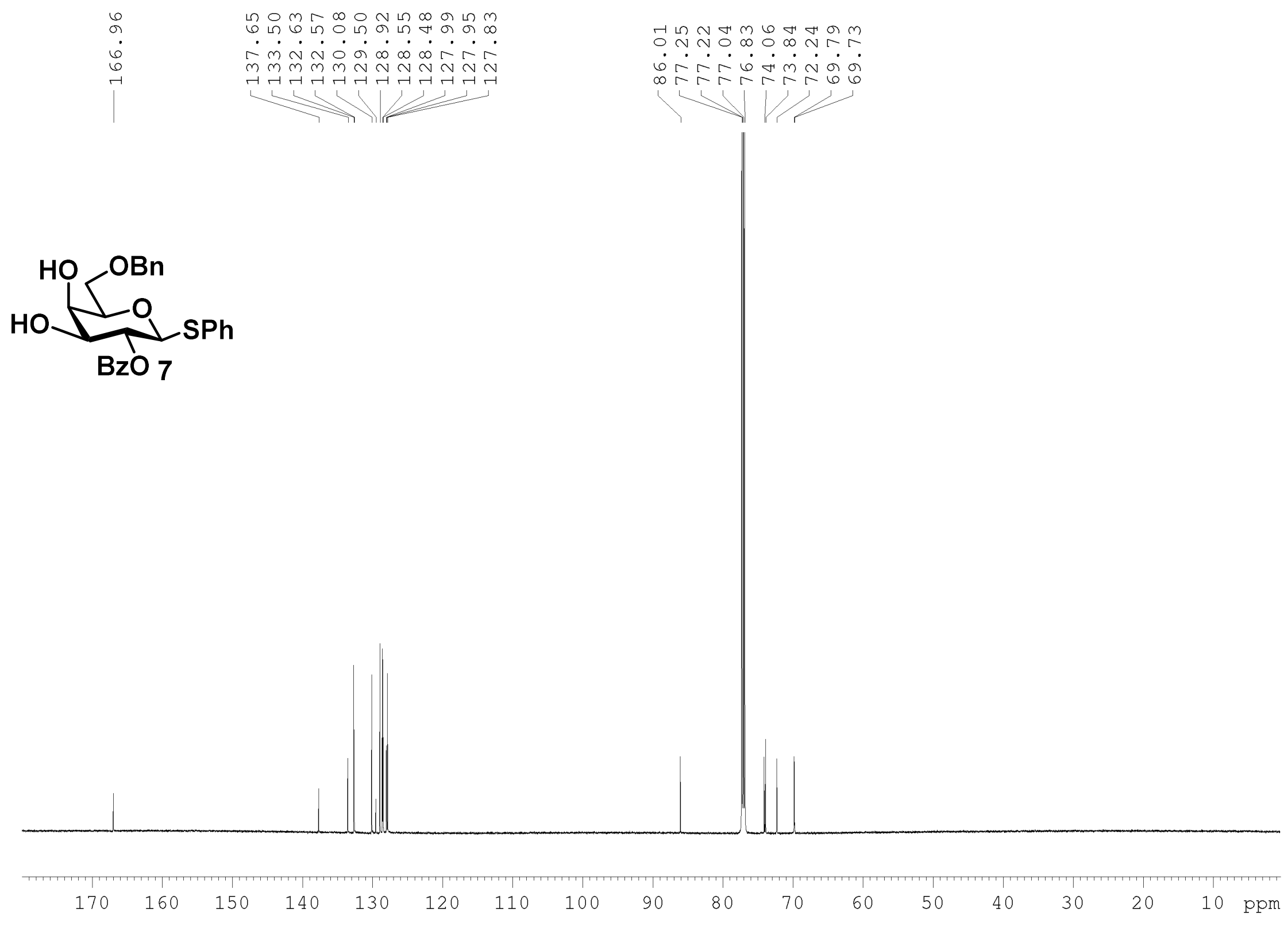


\section{${ }^{1} \mathrm{H} \mathrm{NMR} ; 600 \mathrm{MHz} ; \mathrm{CDCl}_{3}$}

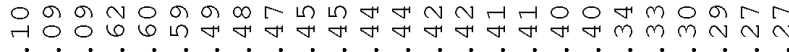

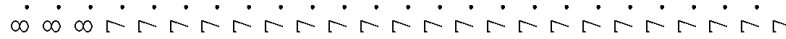

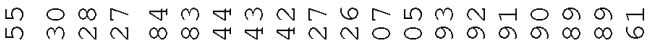

மं மं மं

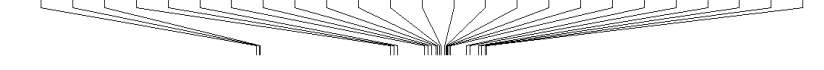

అே

-
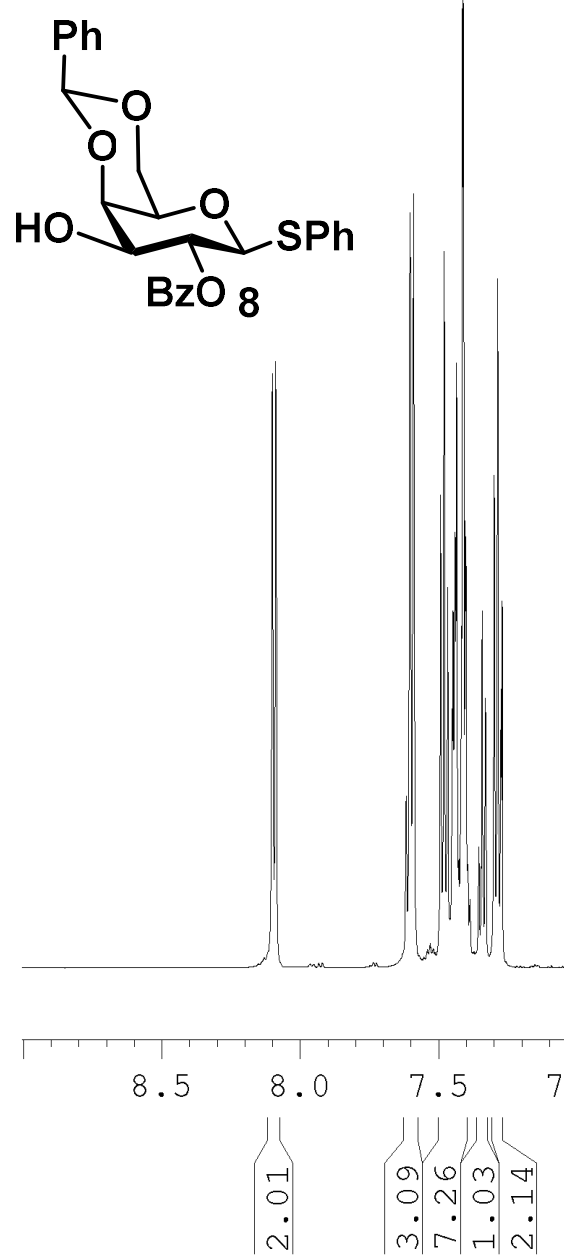

6.5

6.0

$\begin{aligned} & 5.5 \\ & 0 \\ & 0 \\ & 0 \\ & -1\end{aligned} \mid$

5.0

4.5

4. 0

3.5

3.0

2.5

2.0

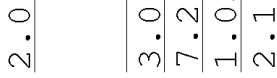

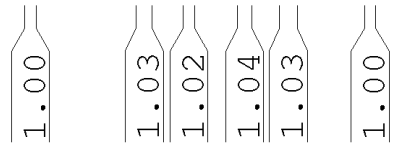

$\left(\begin{array}{l}\infty \\ 0 \\ 0\end{array}\right.$ 
${ }^{13} \mathrm{C} \mathrm{NMR} ; 150 \mathrm{MHz} ; \mathrm{CDCl}_{3}$

$\nabla$
0
0
0
0
$\mid$
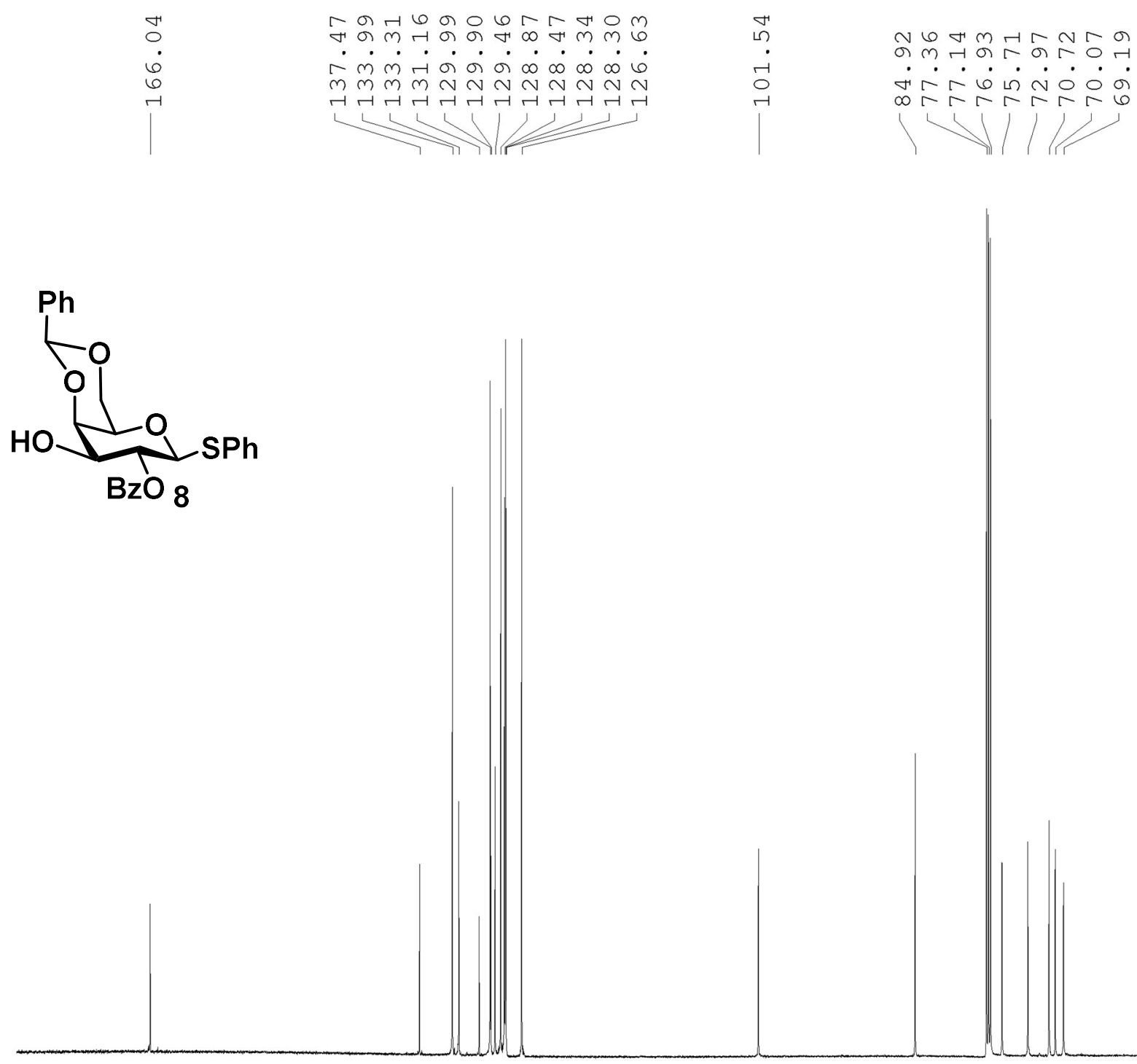

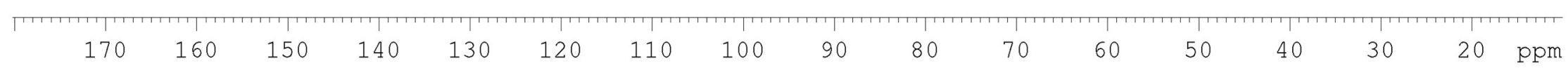




\section{$\underline{{ }^{1} \mathrm{H} N M R ; 600 \mathrm{MHz} ; \mathrm{CDCl}_{3}}$}

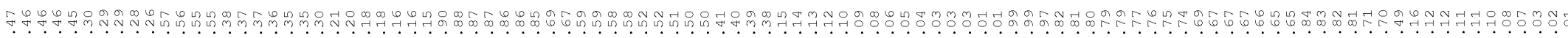

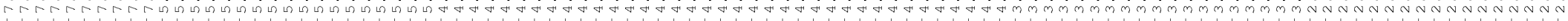

IIIITIIII
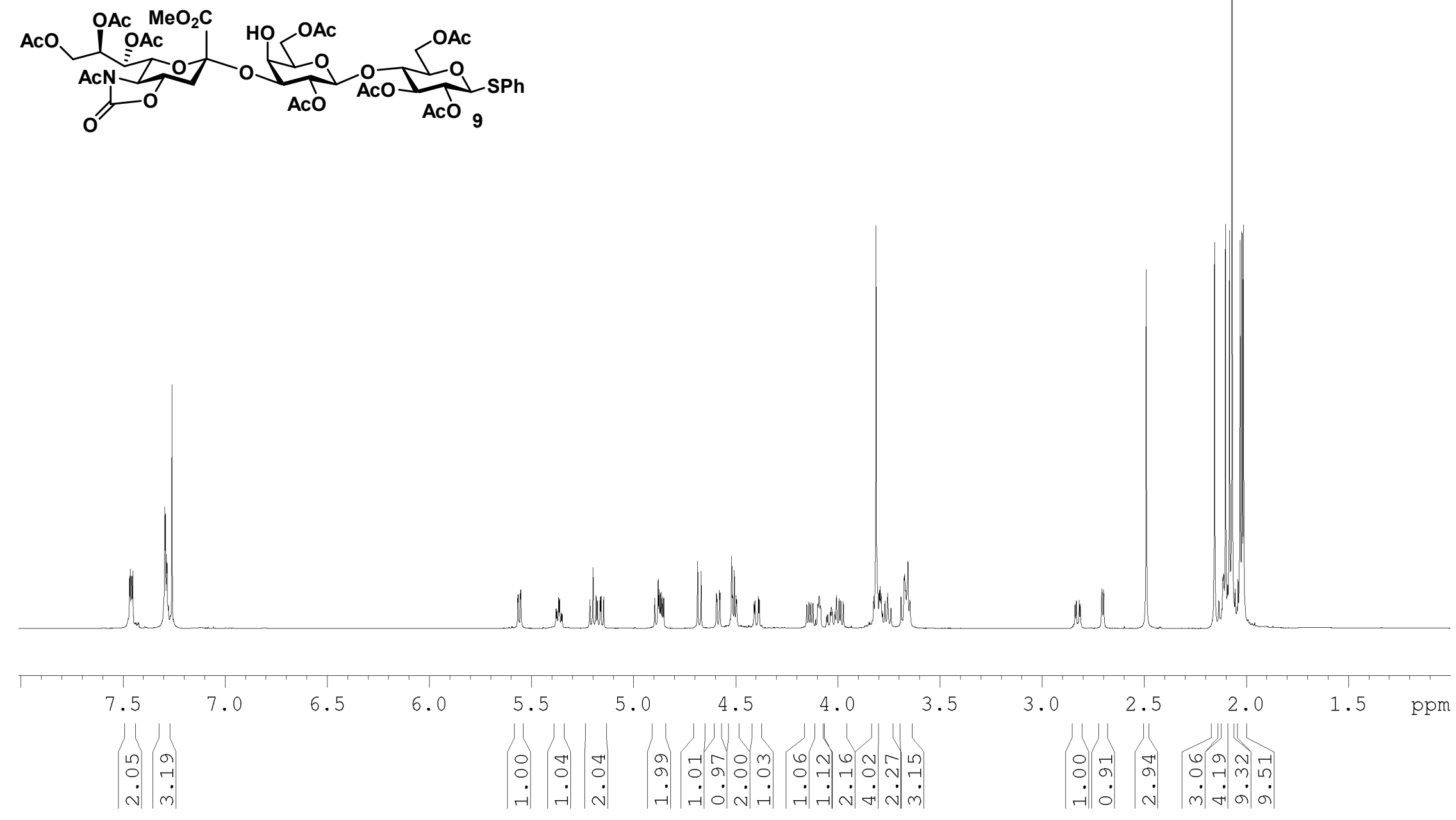
$\underline{{ }^{13} \mathrm{C} \mathrm{NMR} ; 150 \mathrm{MHz} ; \mathrm{CDCl}_{3}}$

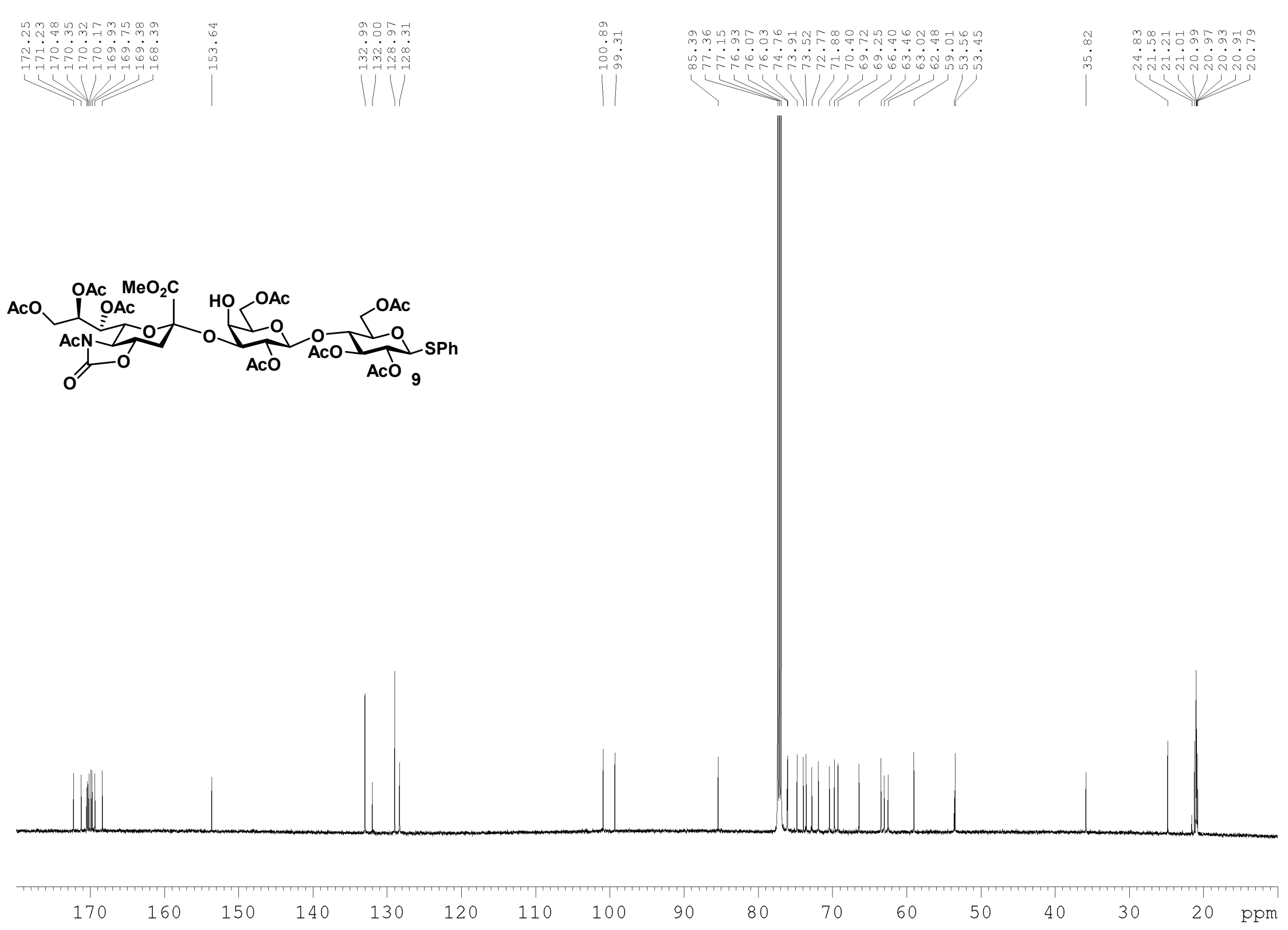




\section{$\underline{\text { DEPT NMR; } 150 \mathrm{MHz} ; \mathrm{CDCl}_{3}}$}

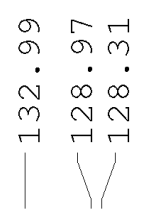

a.

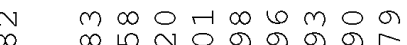

约

L 12
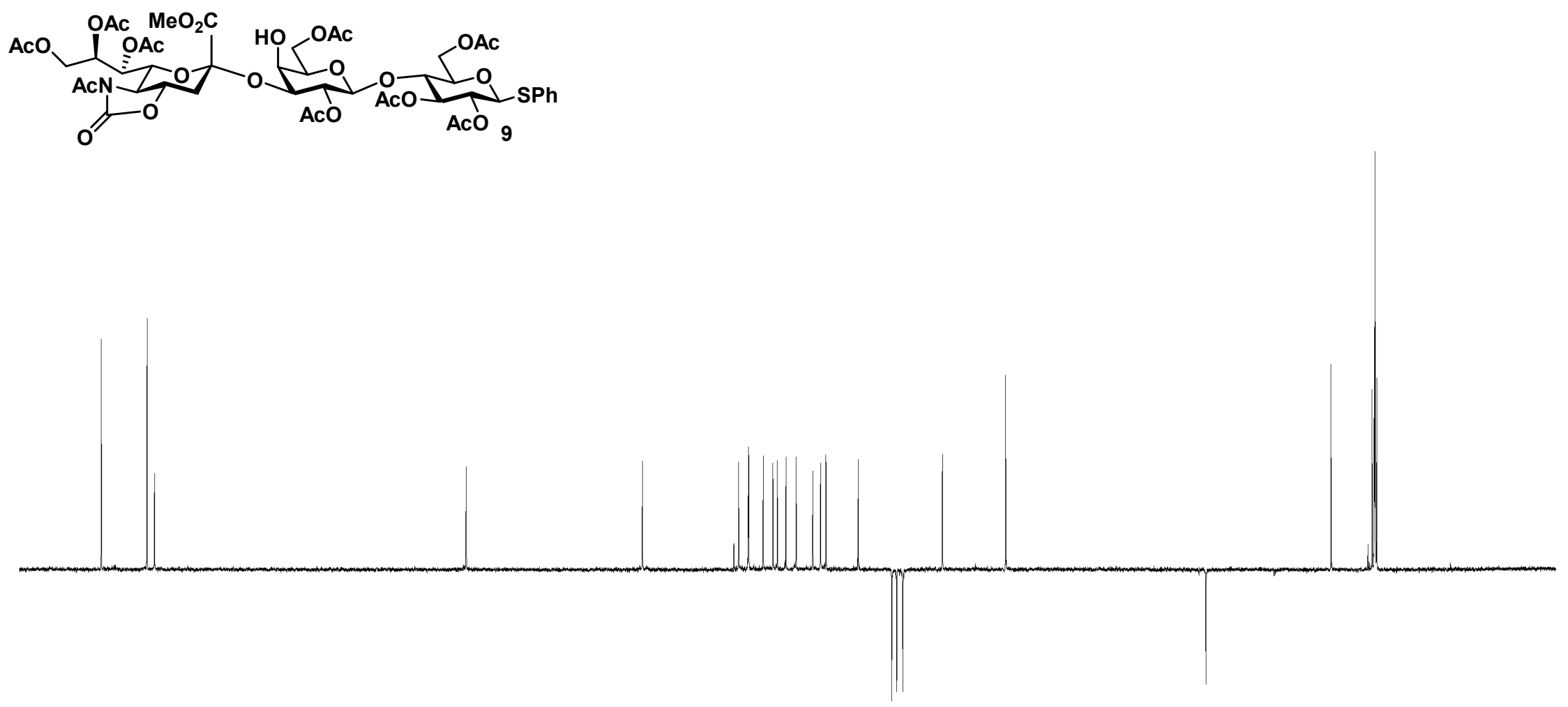

130

120

110

100

90

80

60

50

40

30

20

ppm 


\section{$\underline{\text { COSY NMR; } 600 \mathrm{MHz} ; \mathrm{CDCl}_{3}}$}

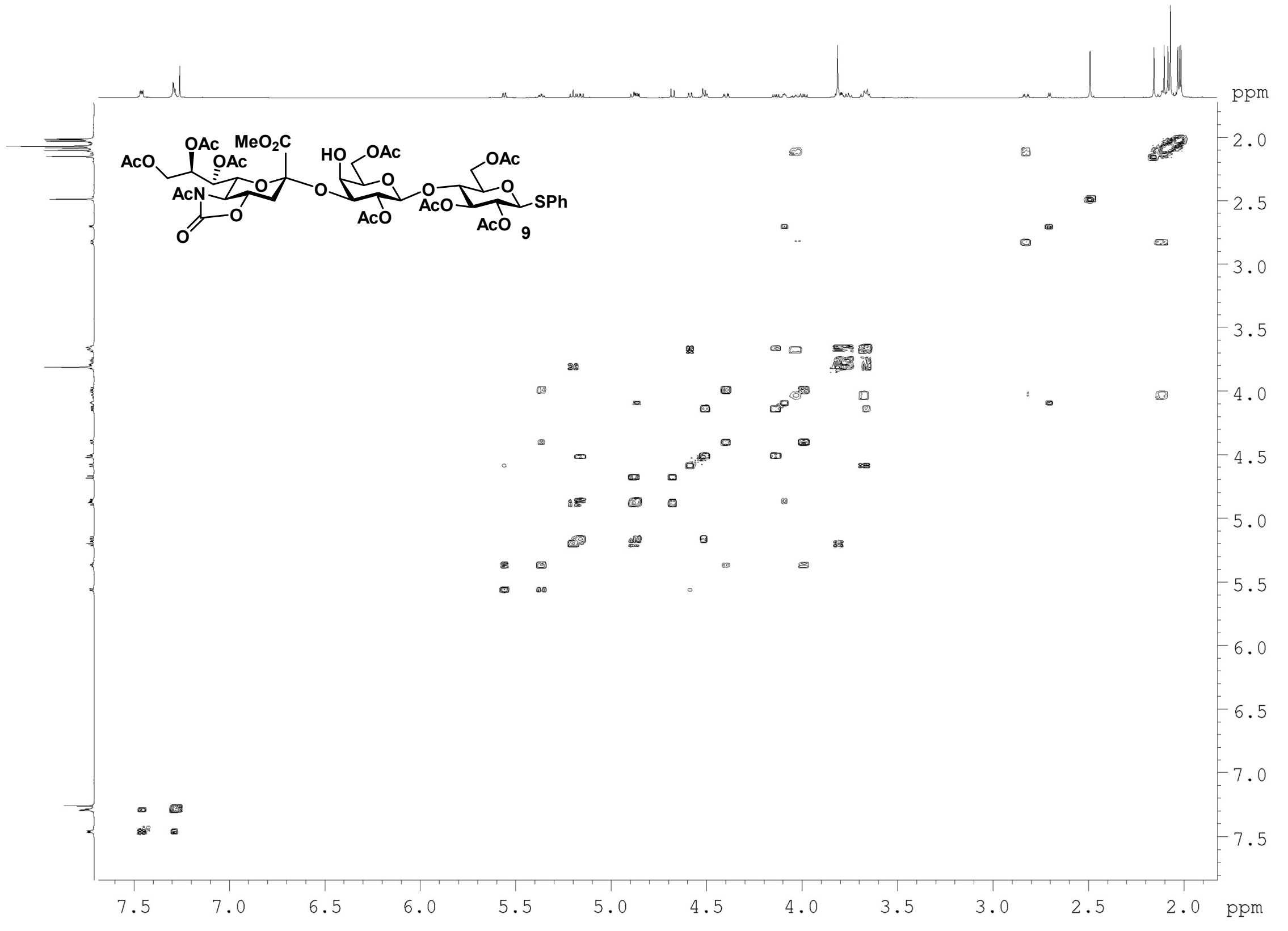




\section{${ }^{1} \mathrm{H} \mathrm{NMR} ; 600 \mathrm{MHz} ; \mathrm{CDCl}_{3}$}

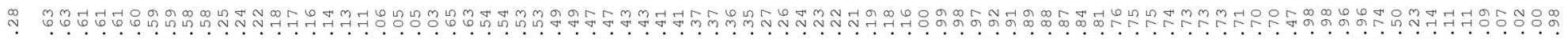
ம்ம் ம்

TIIII

OAc $\mathrm{MeO}_{2} \mathrm{C}$ HO OAC
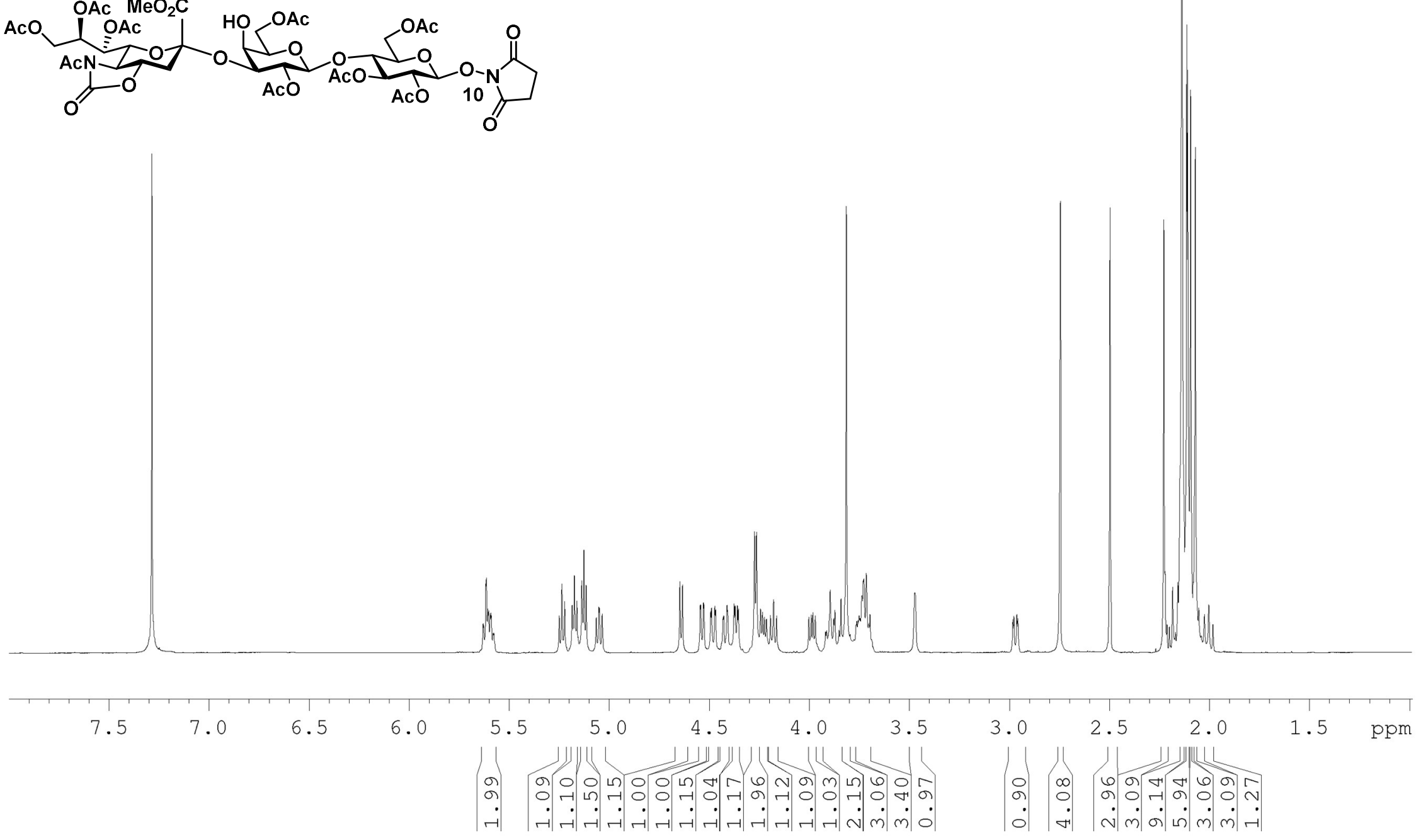
${ }^{13} \mathrm{C} \mathrm{NMR} ; 150 \mathrm{MHz} ; \mathrm{CDCl}_{3}$

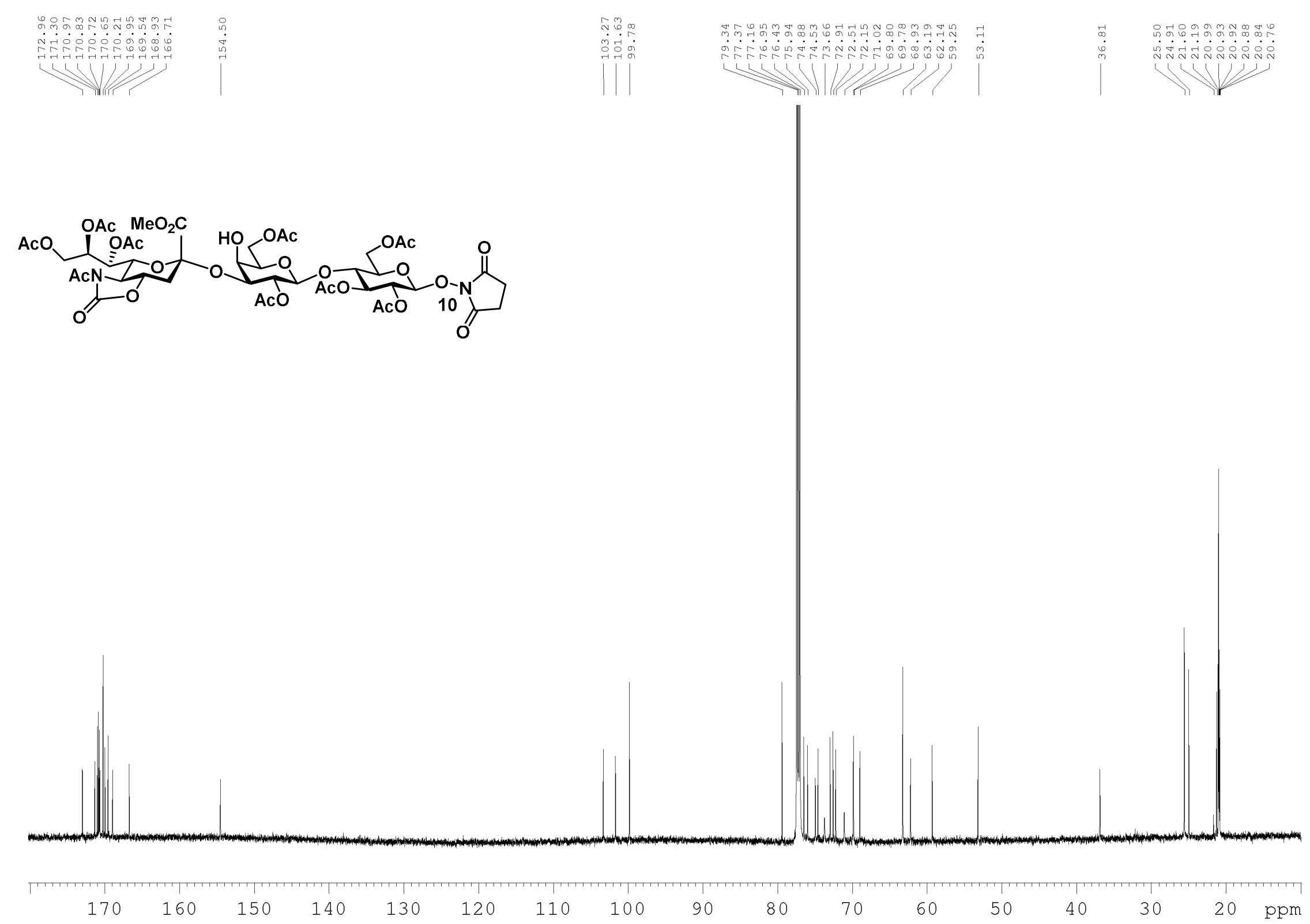


$\underline{\text { DEPT NMR; } 150 \mathrm{MHz} ; \mathrm{CDCl}_{3}}$
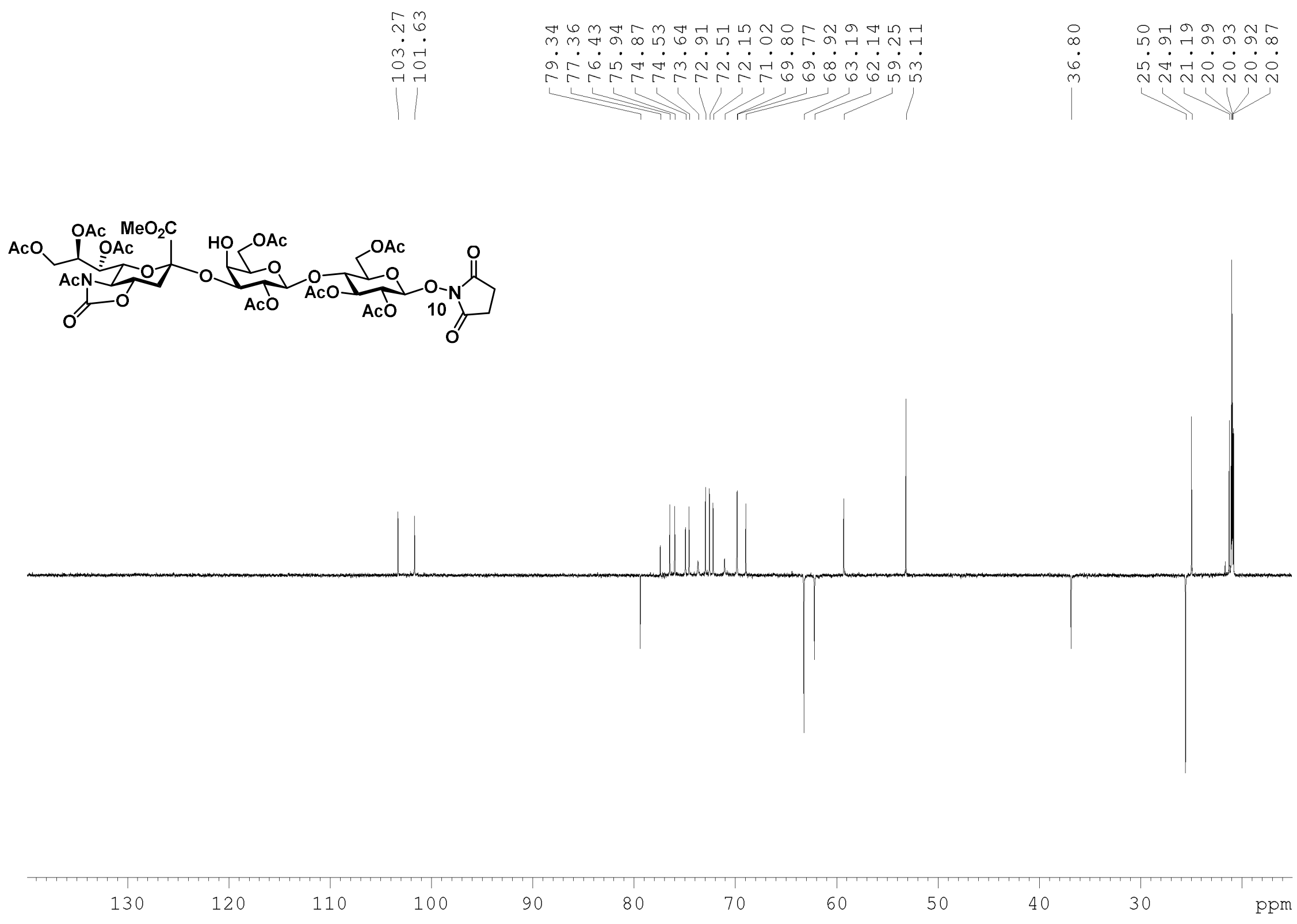


\section{$\underline{\mathrm{COSY} N M R ; 600 \mathrm{MHz} ; \mathrm{CDCl}_{3}}$}

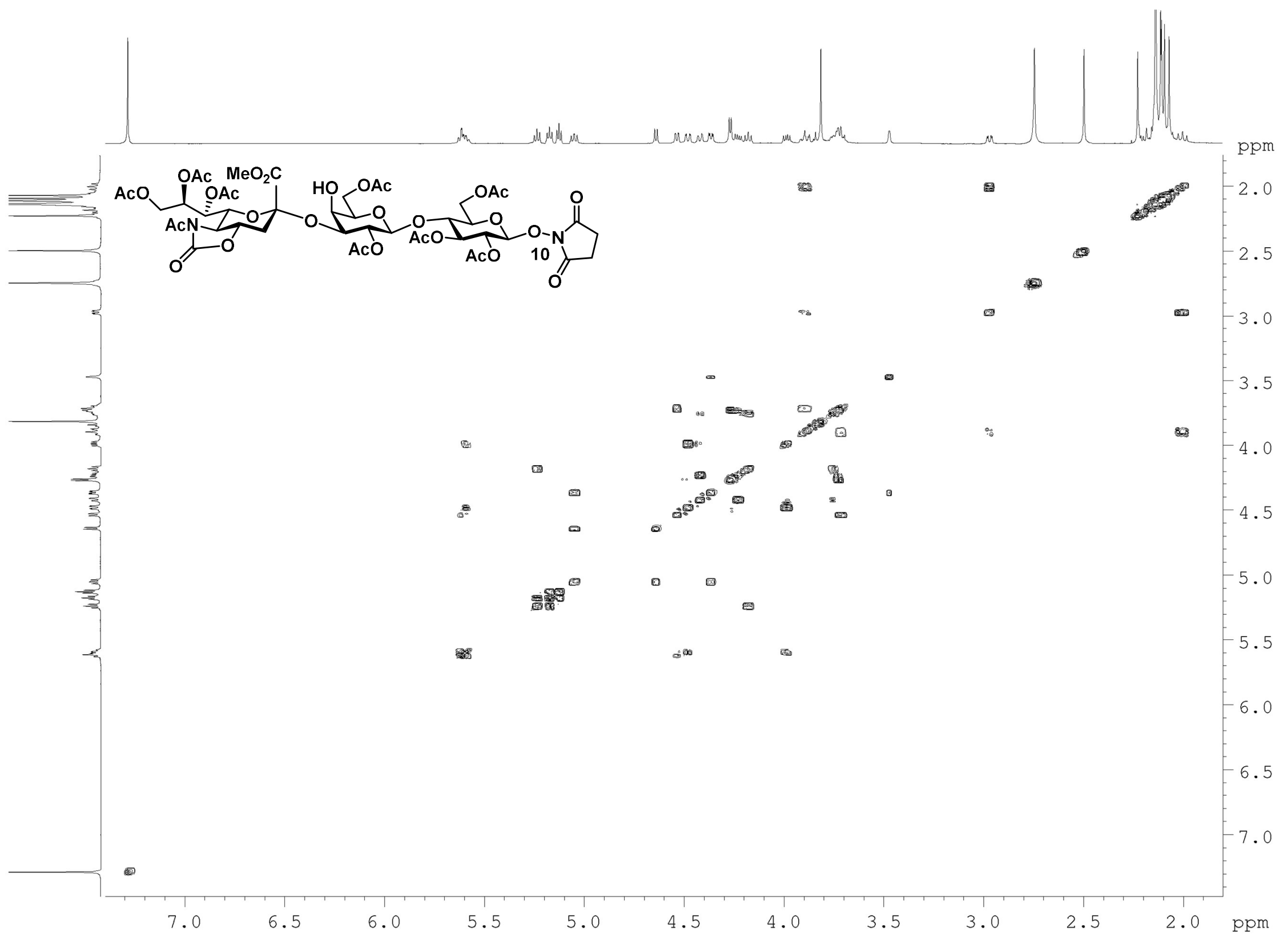




\section{$\underline{{ }^{1} \mathrm{H} \mathrm{NMR} ; 600 \mathrm{MHz} ; \mathrm{CDCl}_{3}}$}

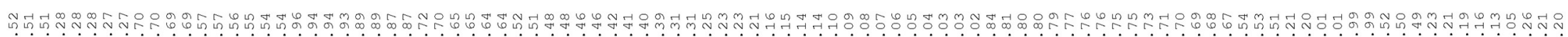

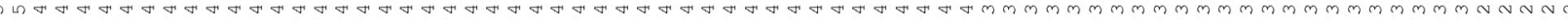

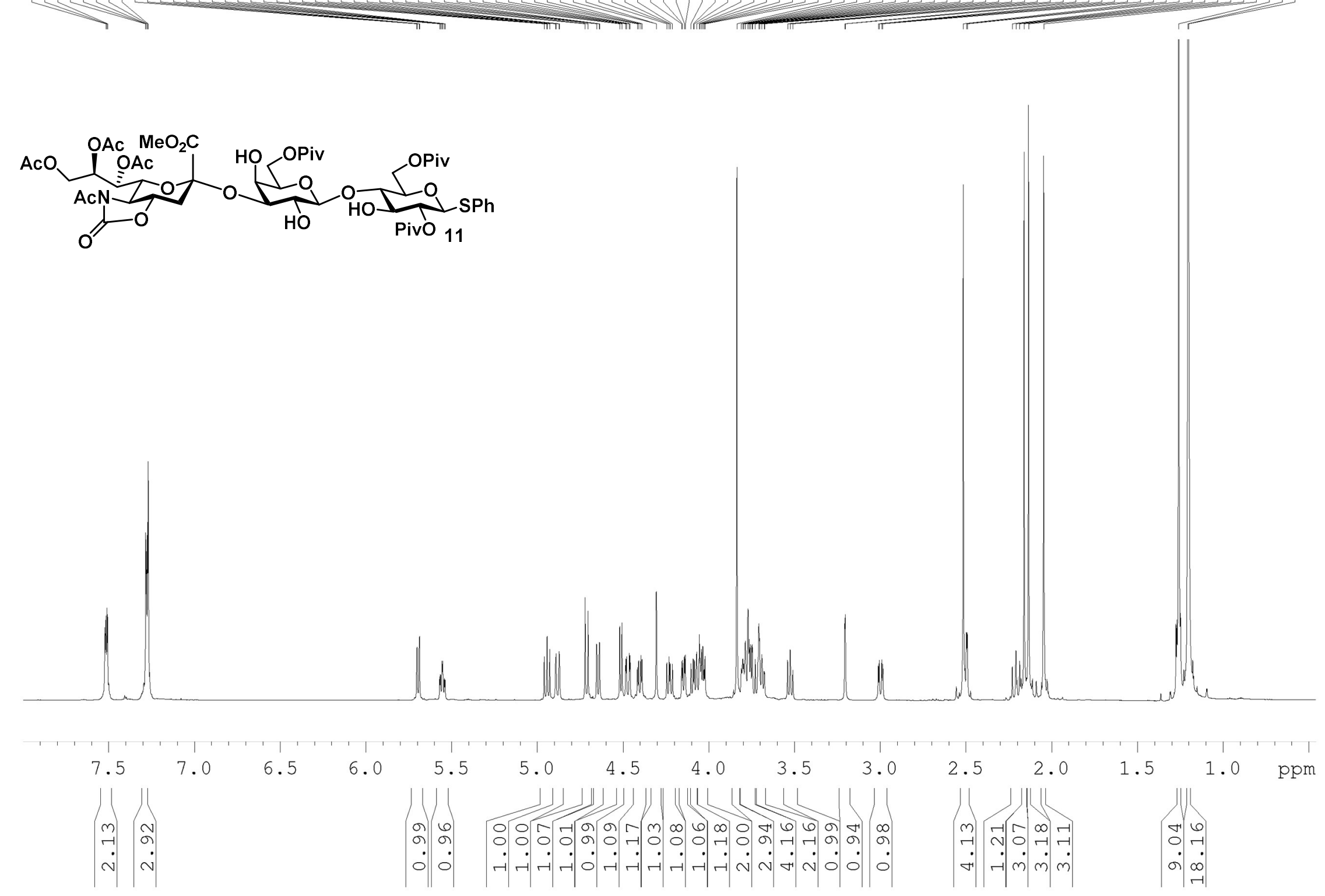


${ }^{13} \mathrm{C} \mathrm{NMR} ; 150 \mathrm{MHz} ; \mathrm{CDCl}_{3}$
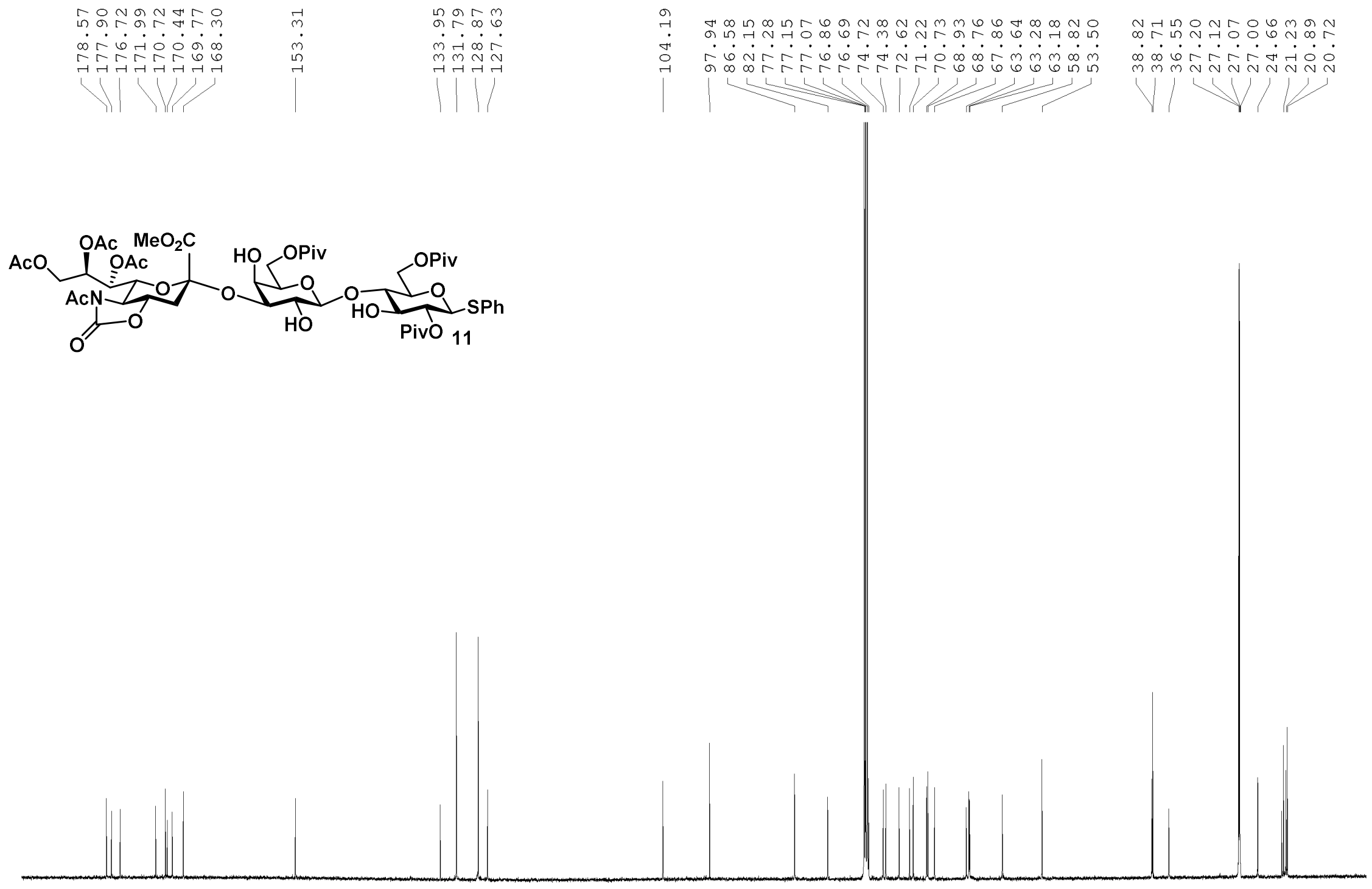

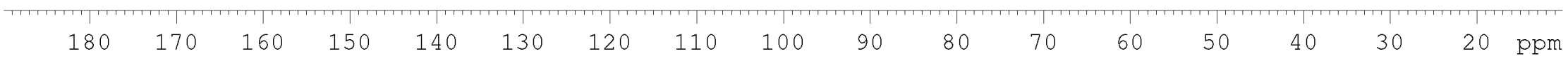


$\underline{\text { DEPT NMR; } 150 \mathrm{MHz} ; \mathrm{CDCl}_{3}}$

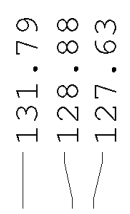

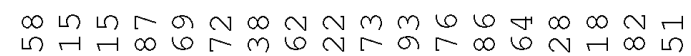

$\cdot \cdot \cdot \cdot \cdot \cdot \cdot \cdot \cdot \cdot \cdot \cdot \cdot \cdot \cdot \cdot \cdot \cdot \cdot \cdot$

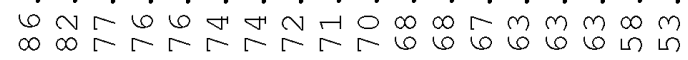

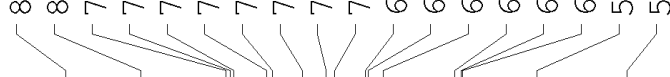
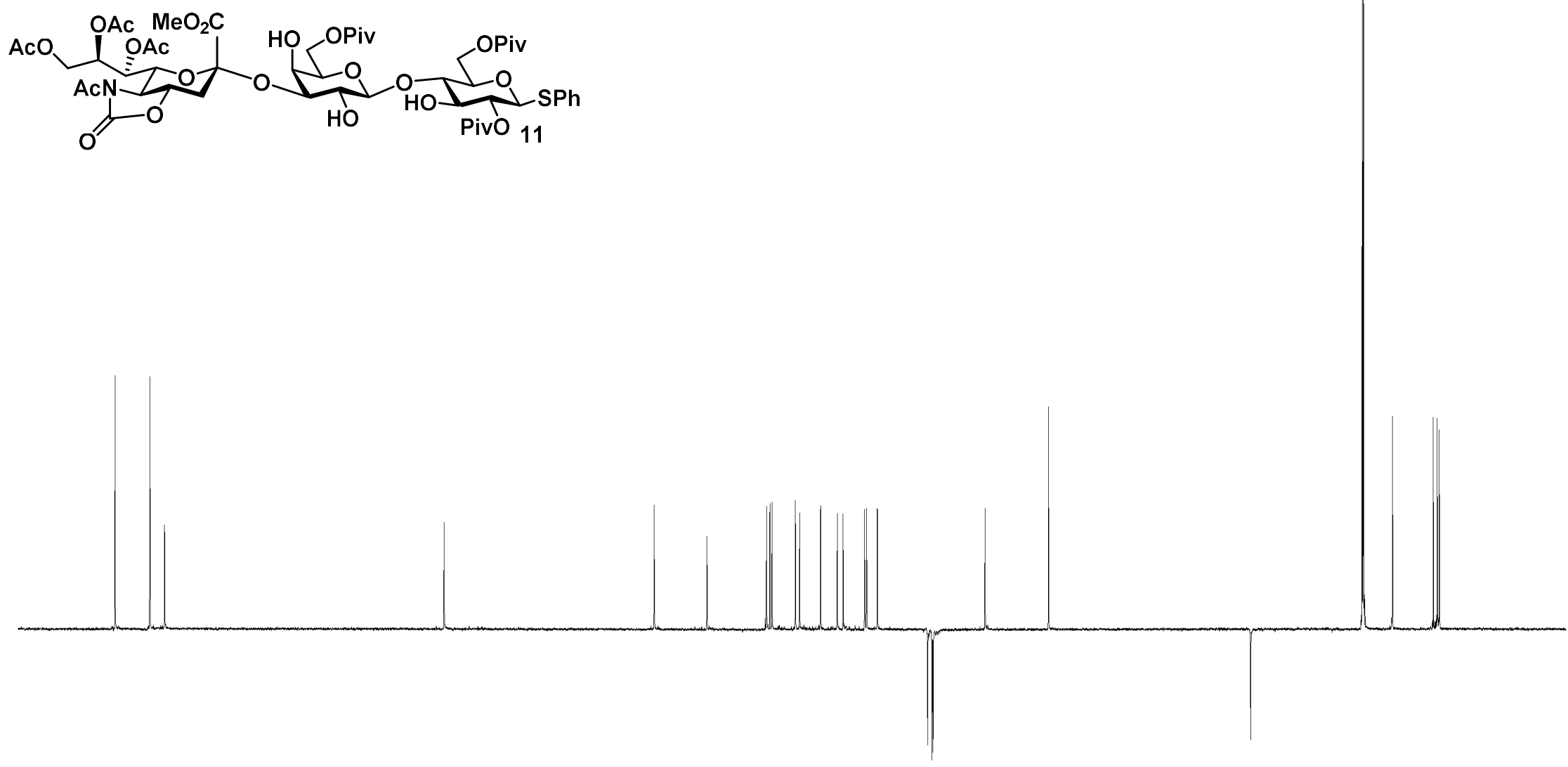


\section{$\underline{\text { COSY NMR; } 600 \mathrm{MHz} ; \mathrm{CDCl}_{3}}$}

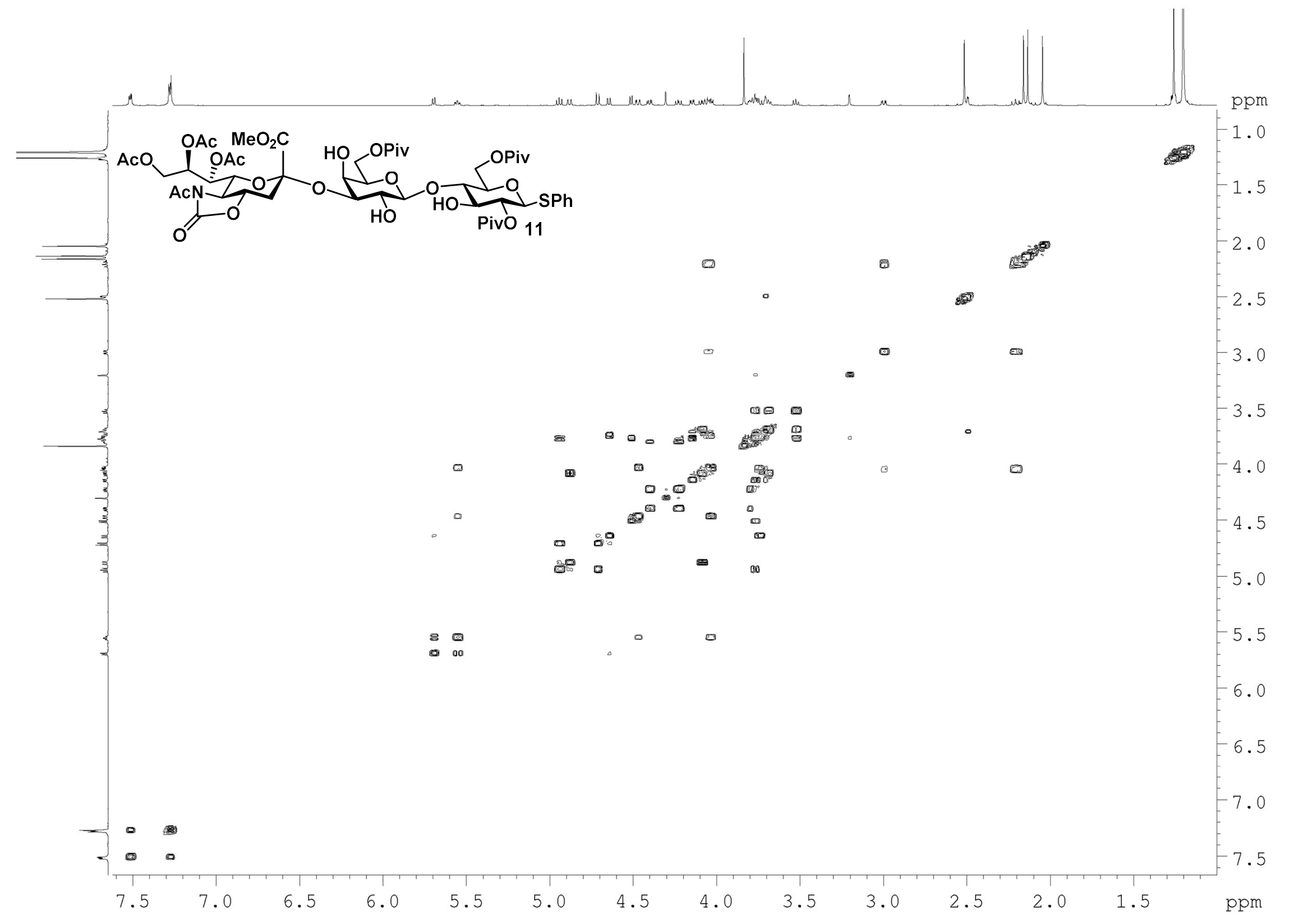




\section{$\underline{{ }^{1} \mathrm{H} N M R ; 600 \mathrm{MHz} ; \mathrm{CDCl}_{3}}$}

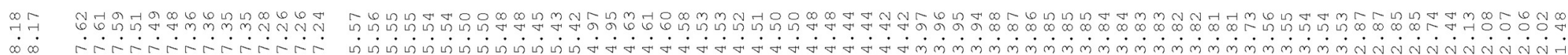

(1)
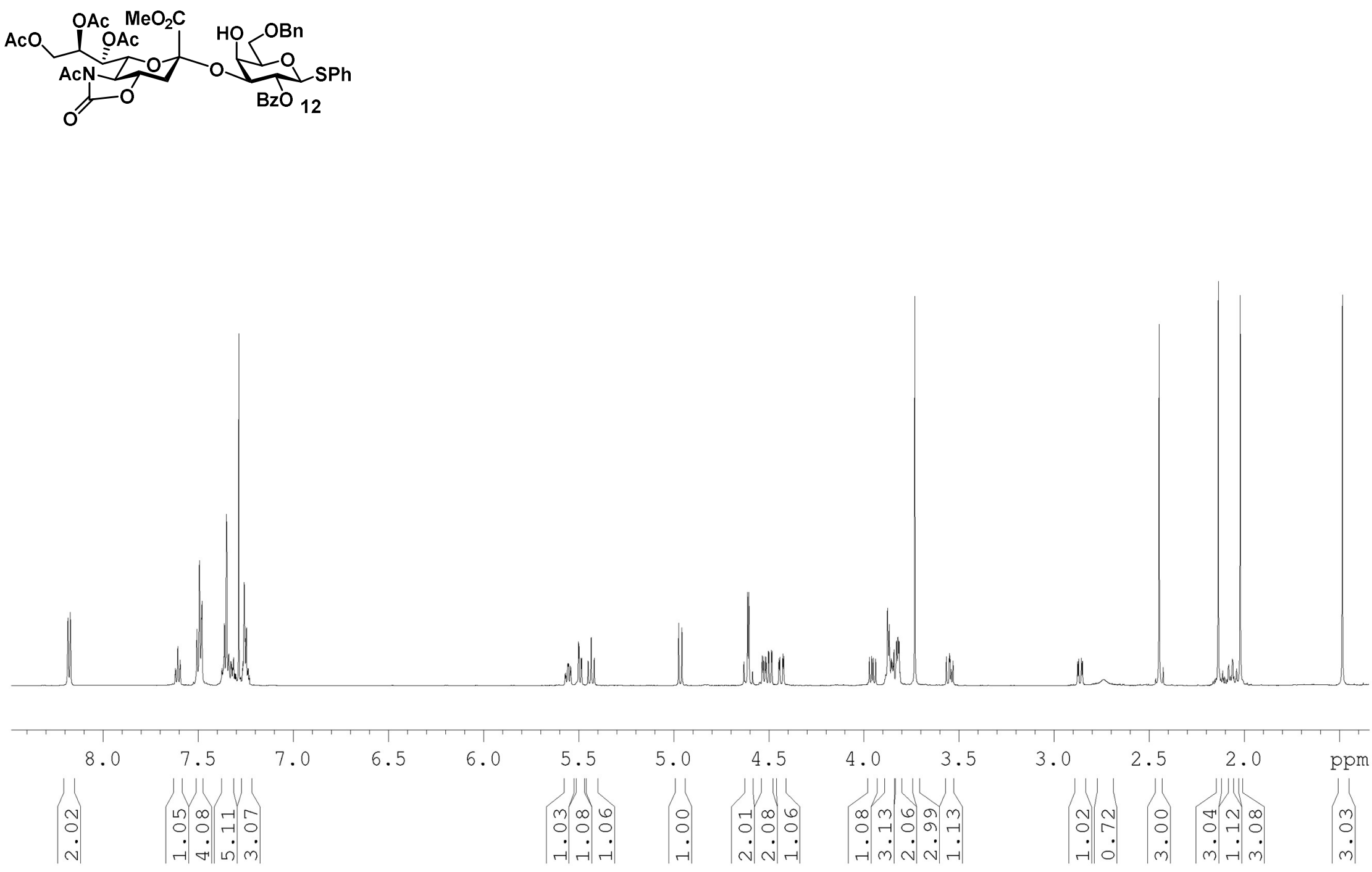


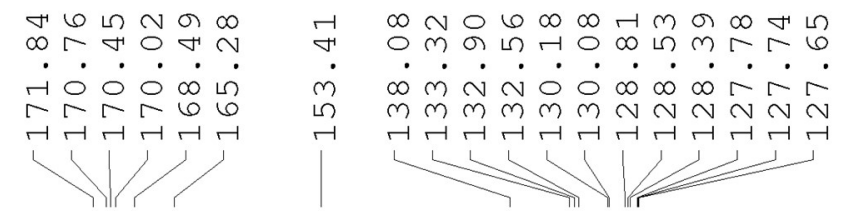

(

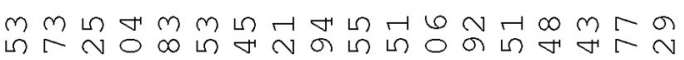
?.

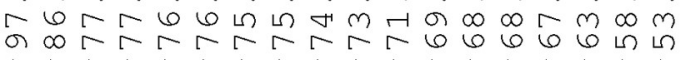
$\longrightarrow$
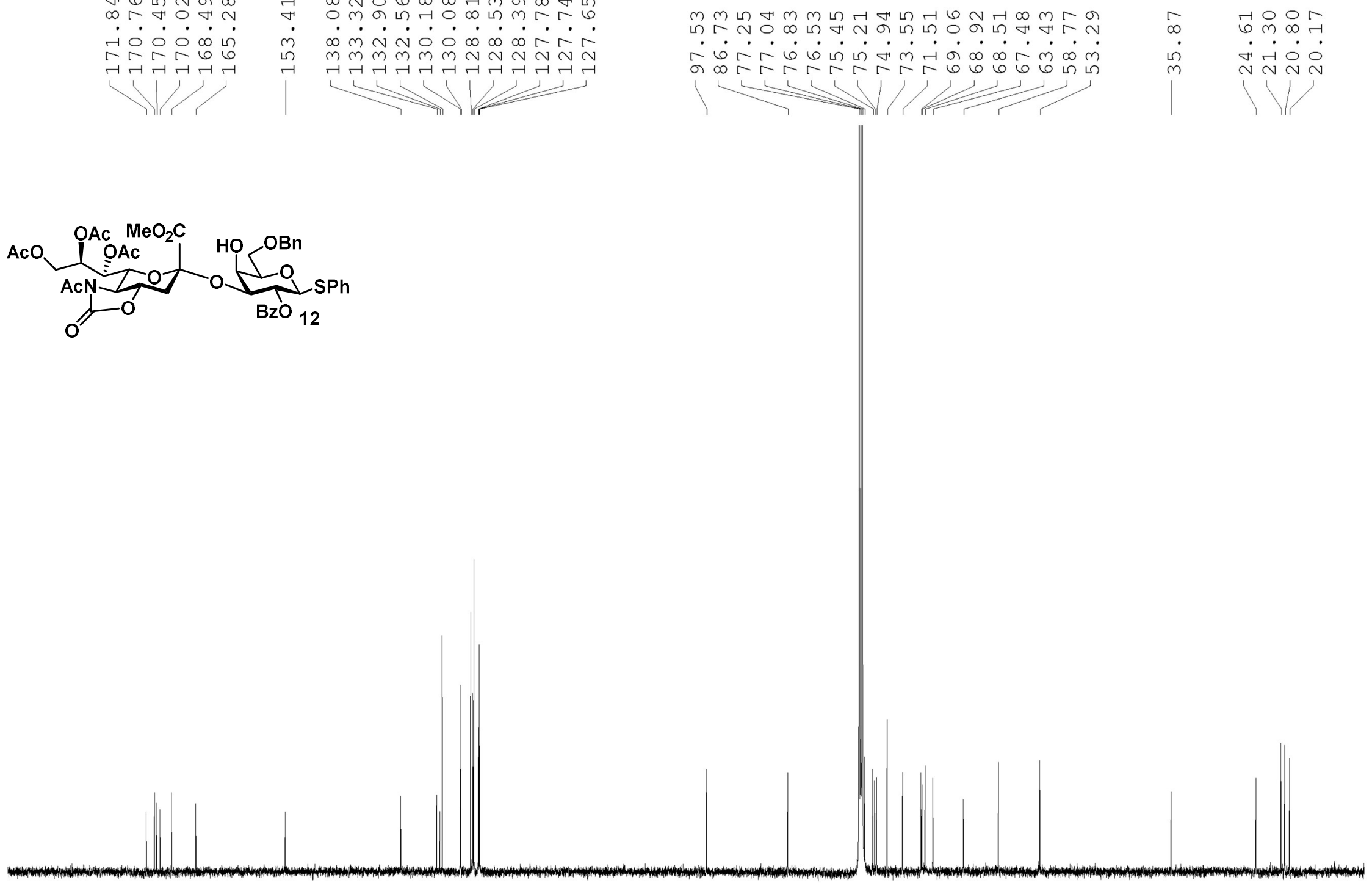


\section{$\underline{\text { DEPT NMR; } 150 \mathrm{MHz} ; \mathrm{CDCl}_{3}}$}

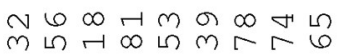

$\dot{m} \dot{N} \dot{0} \infty \infty \dot{\sim} \dot{\sim}$

$m m m \sim N N N \sim$

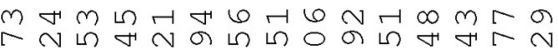

bங்ம்

$4<$

$\longrightarrow$

क

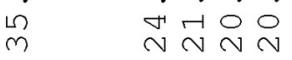
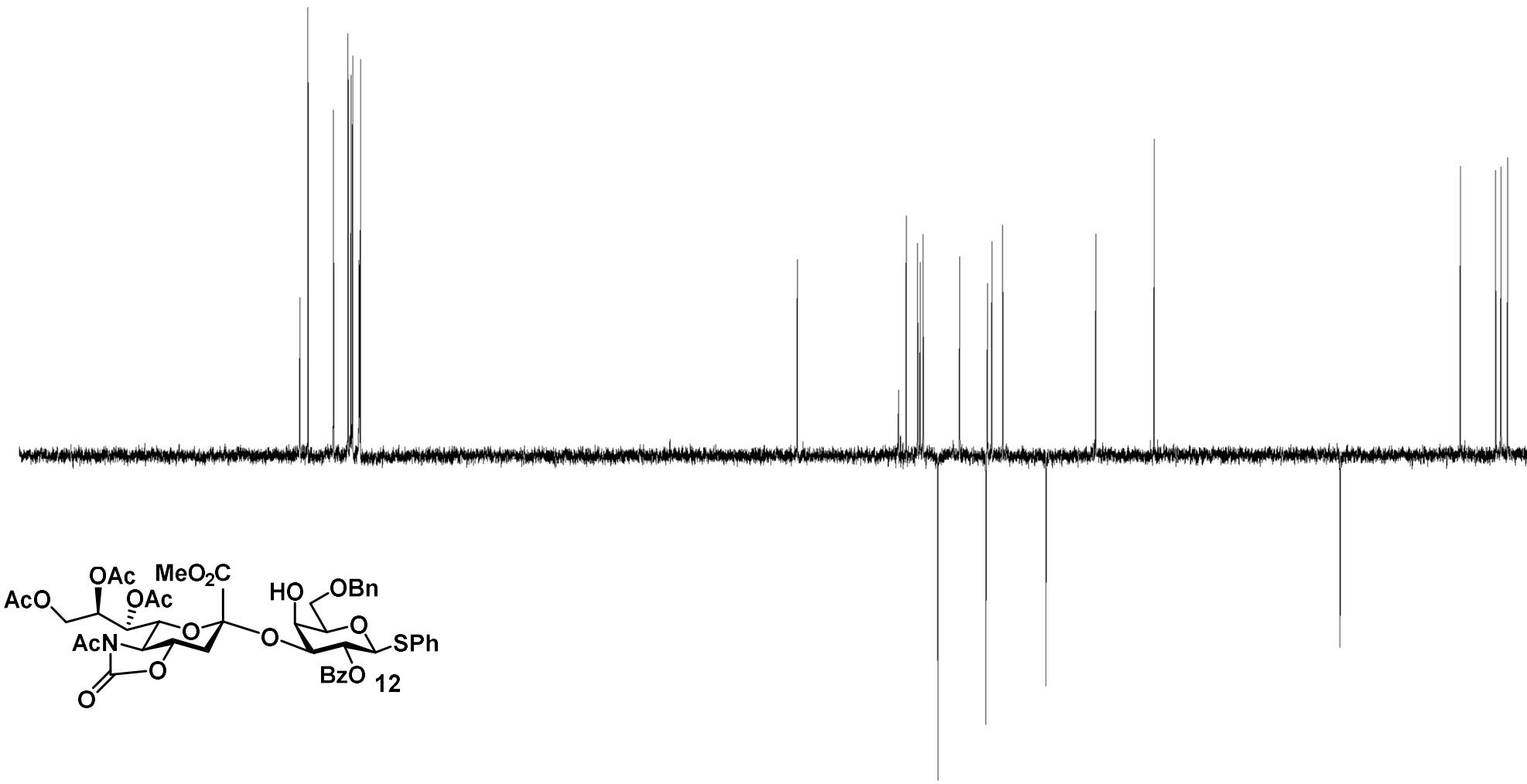

$140 \quad 130$




\section{$\underline{\text { COSY NMR; } 600 \mathrm{MHz} ; \mathrm{CDCl}_{3}}$}

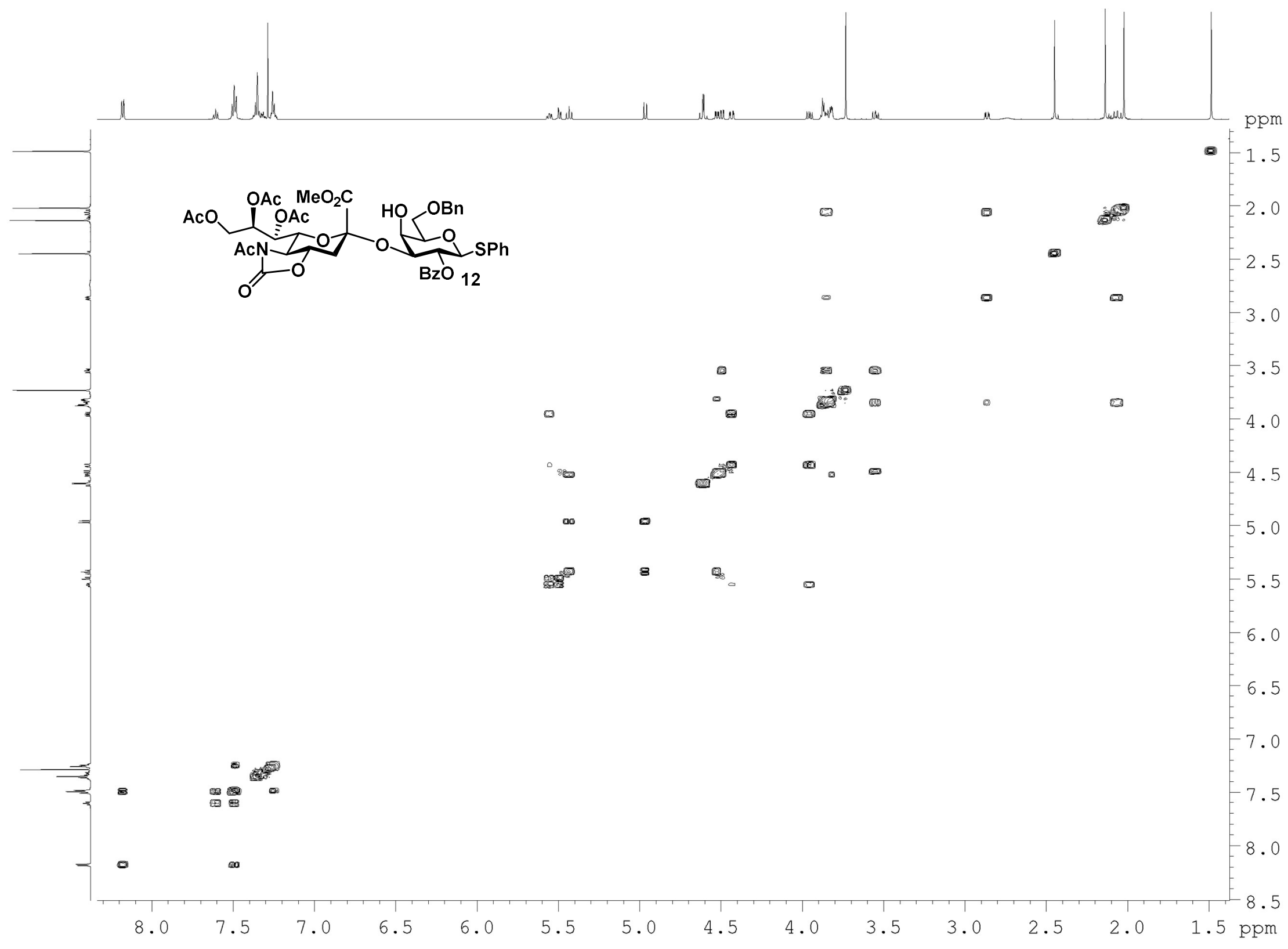


$\underline{{ }^{1} \mathrm{H} \text { NMR; } 600 \mathrm{MHz} ; \mathrm{CDCl}_{3}}$
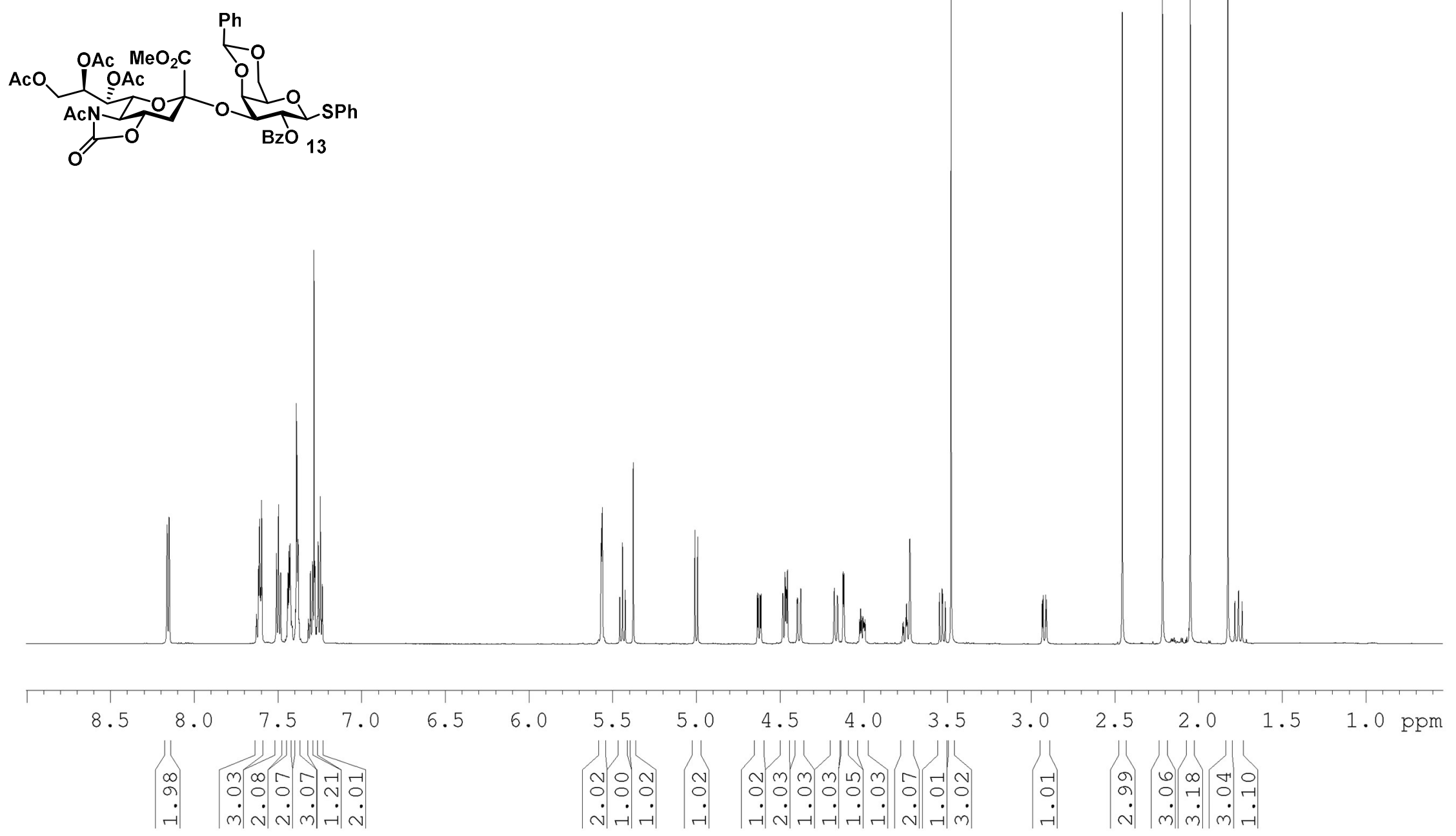

S43 
${ }^{13} \mathrm{C} \mathrm{NMR;} 150 \mathrm{MHz} ; \mathrm{CDCl}_{3}$

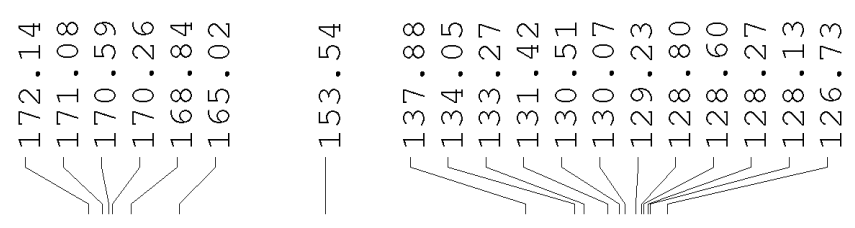

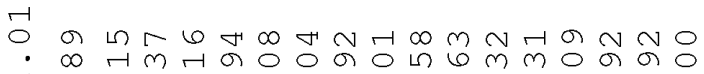

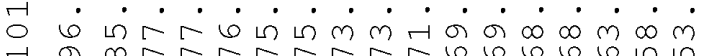

6

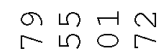

मंत्रें
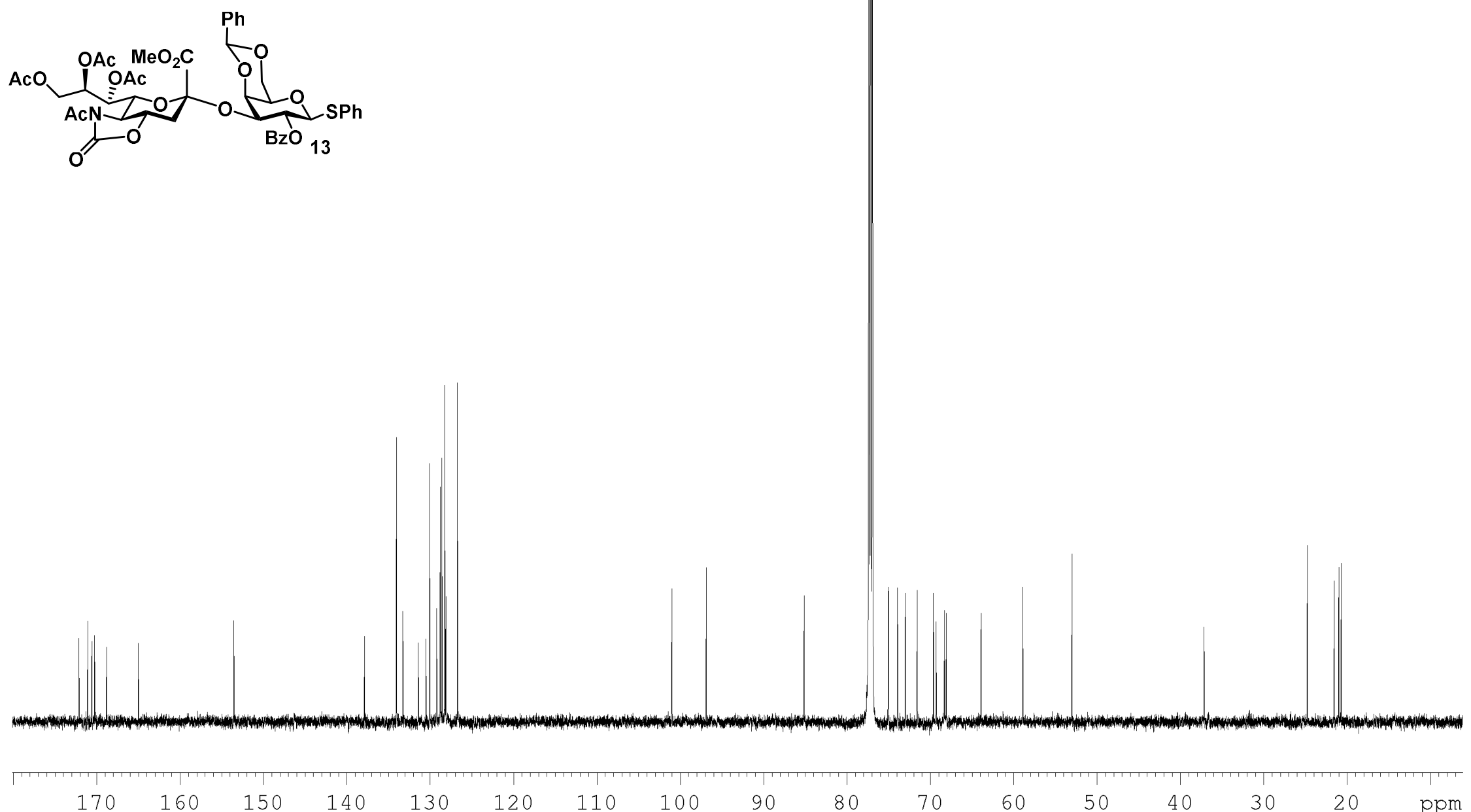

160

150

140

130

120

110

100

90

80

70

60

50

40

30

20

ppm 


\section{$\underline{\text { DEPT NMR; } 150 \mathrm{MHz} ; \mathrm{CDCl}_{3}}$}

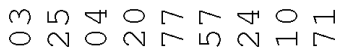

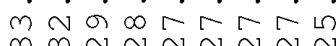

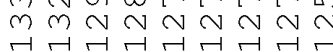

\%

पर्या

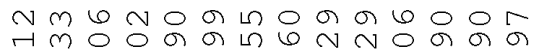

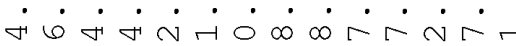

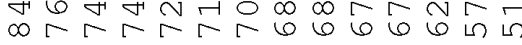

, l

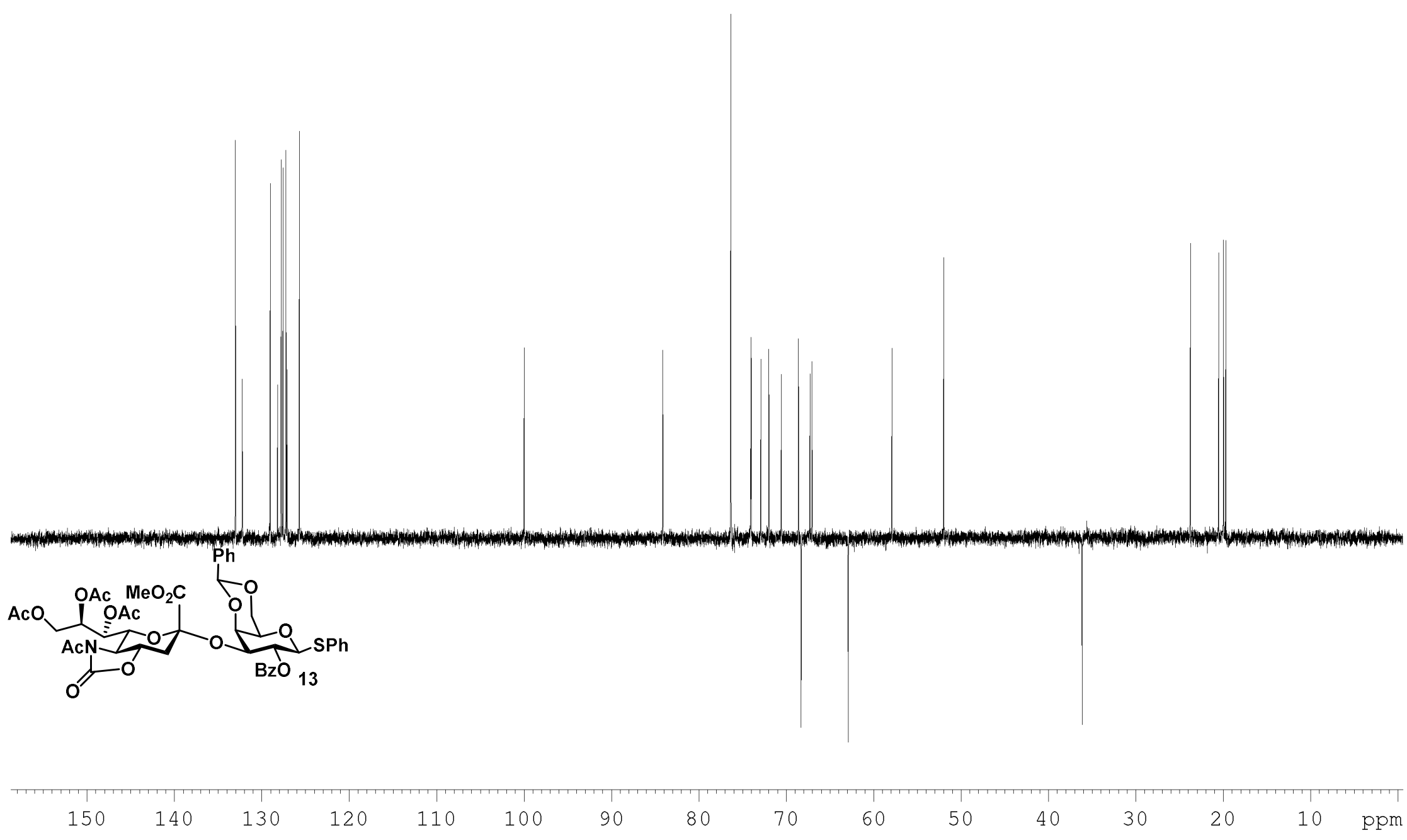


COSY NMR; $600 \mathrm{MHz} ; \mathrm{CDCl}_{3}$

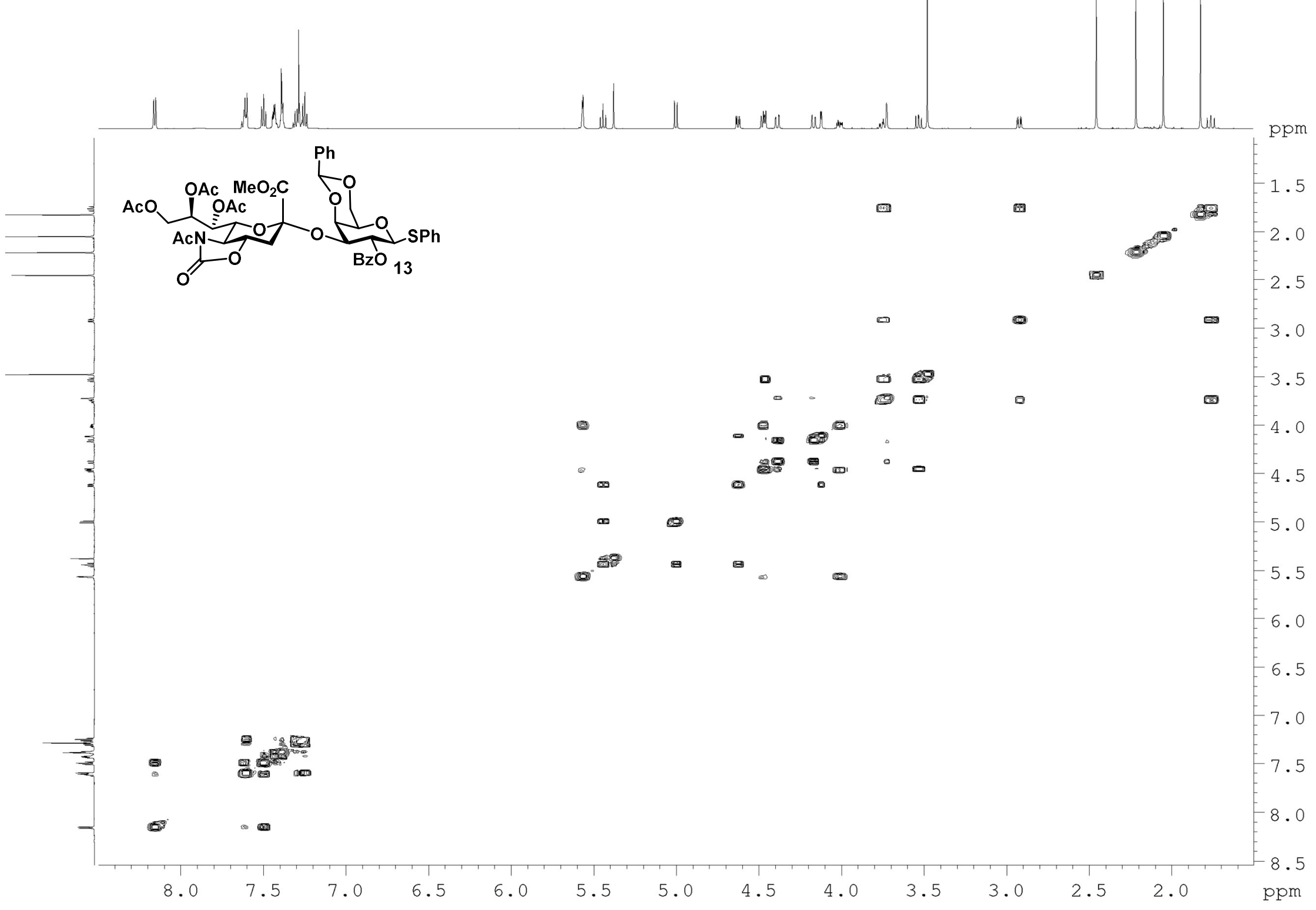




\section{${ }^{1} \mathrm{H}$ NMR; $600 \mathrm{MHz} ; \mathrm{CDCl}_{3}$}

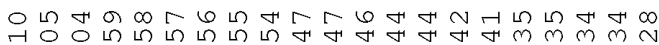

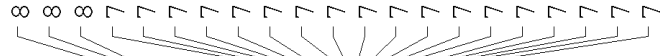

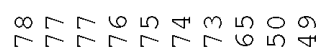

மिं

以 以

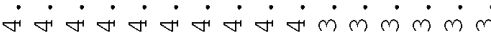

L

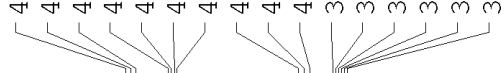



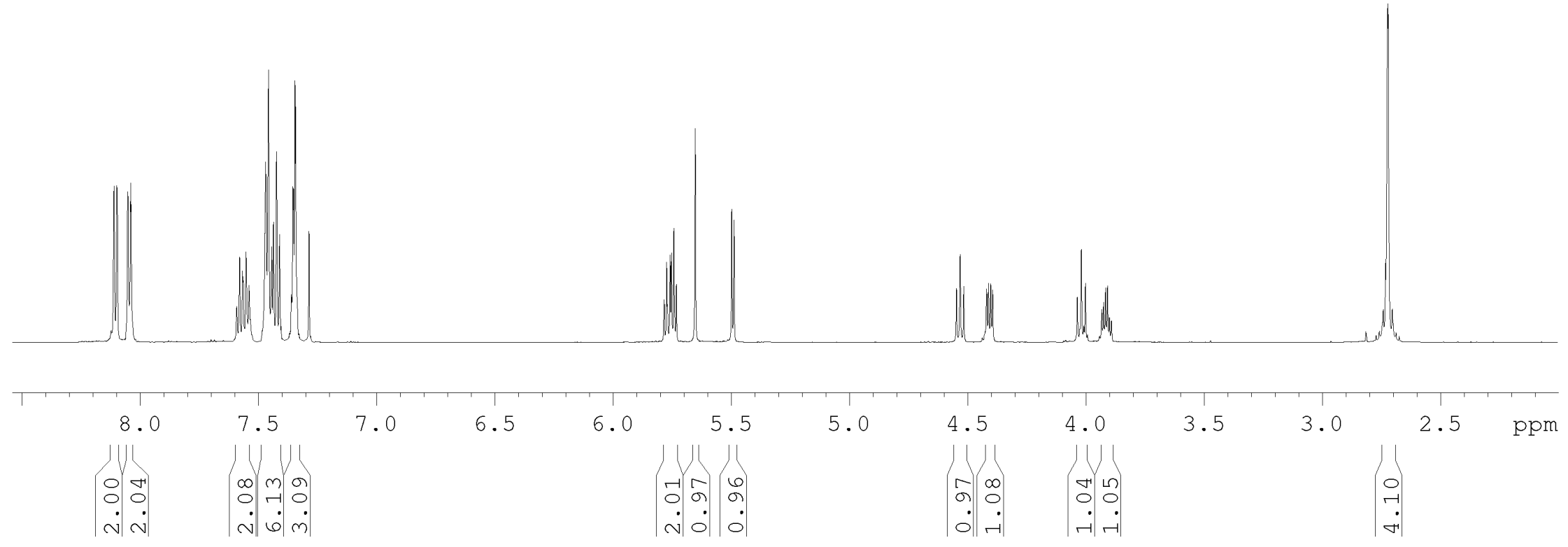




\section{${ }^{13} \mathrm{C} \mathrm{NMR} ; 150 \mathrm{MHz} ; \mathrm{CDCl}_{3}$}
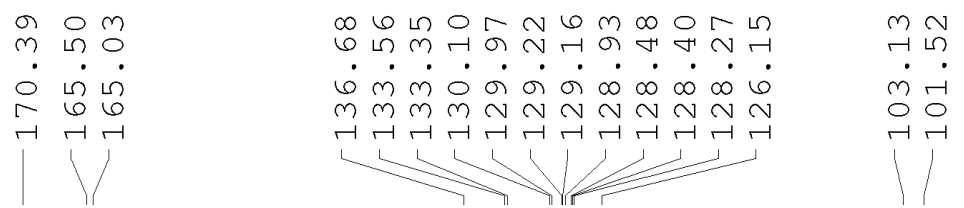

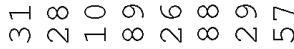

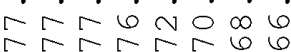
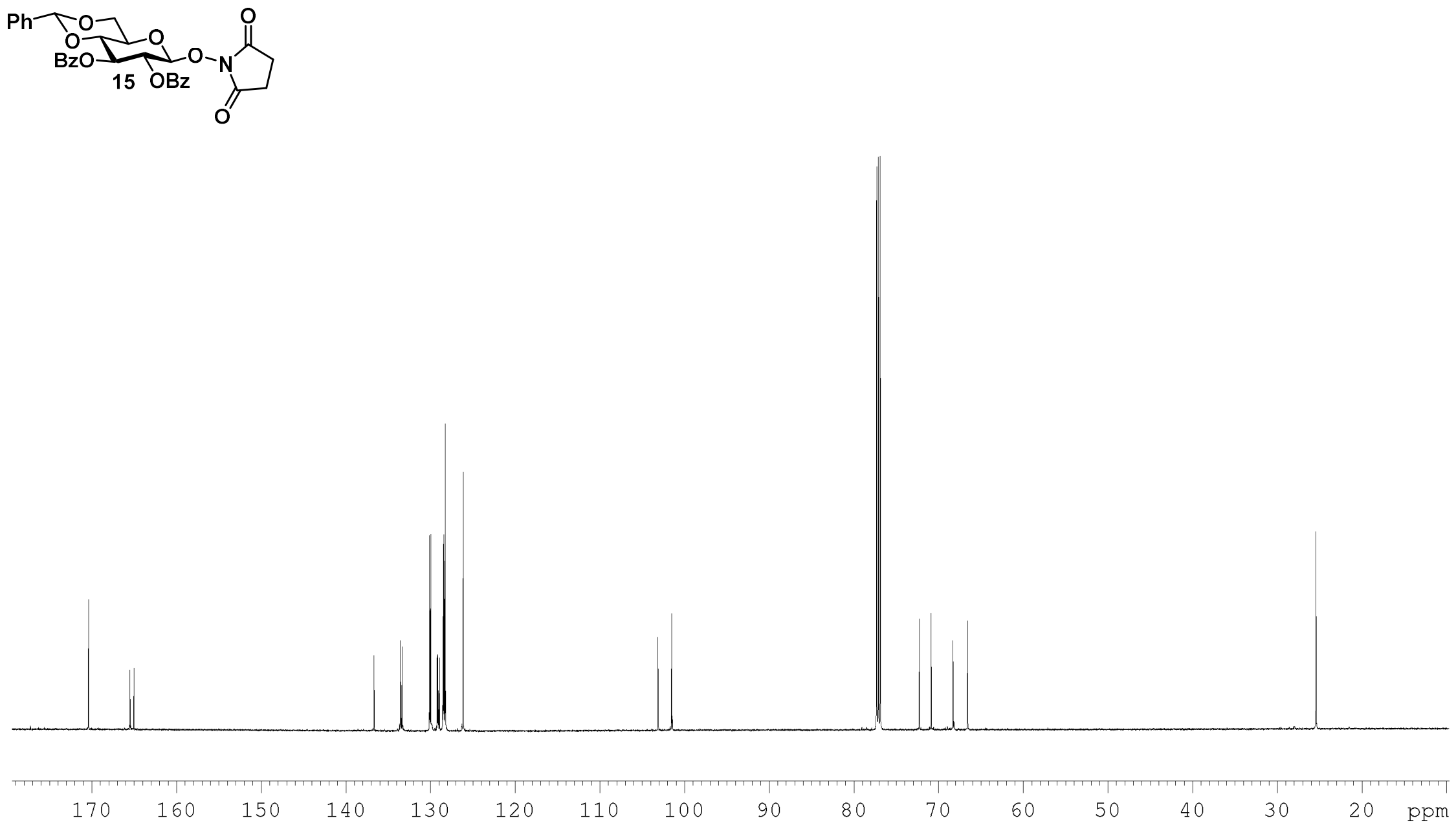


\section{${ }^{1} \mathrm{H} \mathrm{NMR} ; 600 \mathrm{MHz} ; \mathrm{CDCl}_{3}$}

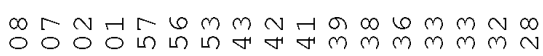

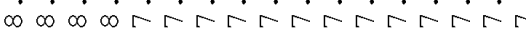

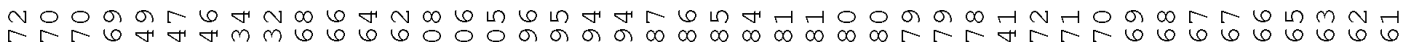
10

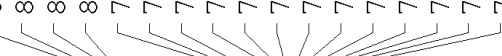

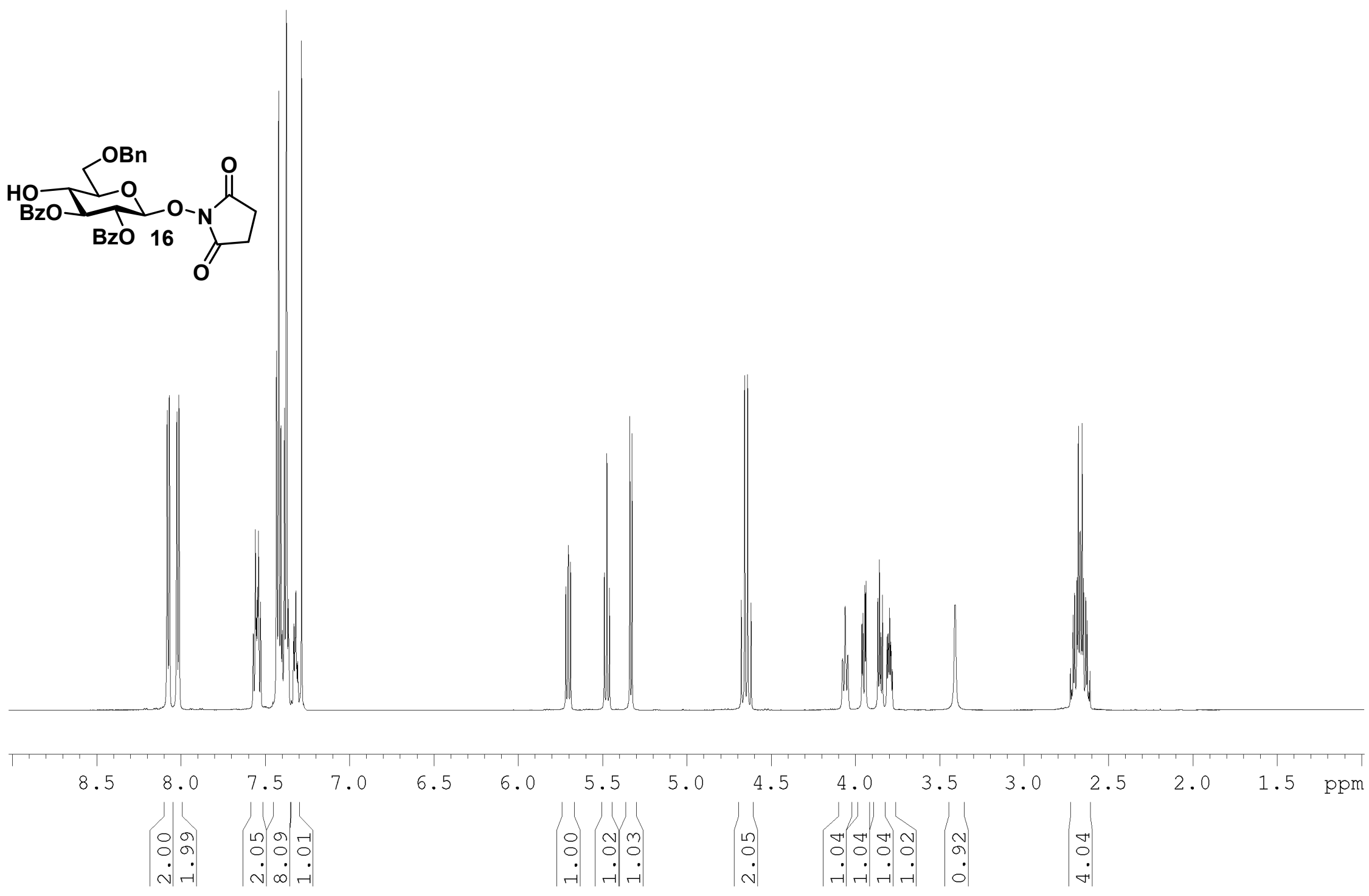


$\underline{{ }^{13} \mathrm{C} \mathrm{NMR} ; 150 \mathrm{MHz} ; \mathrm{CDCl}_{3}}$

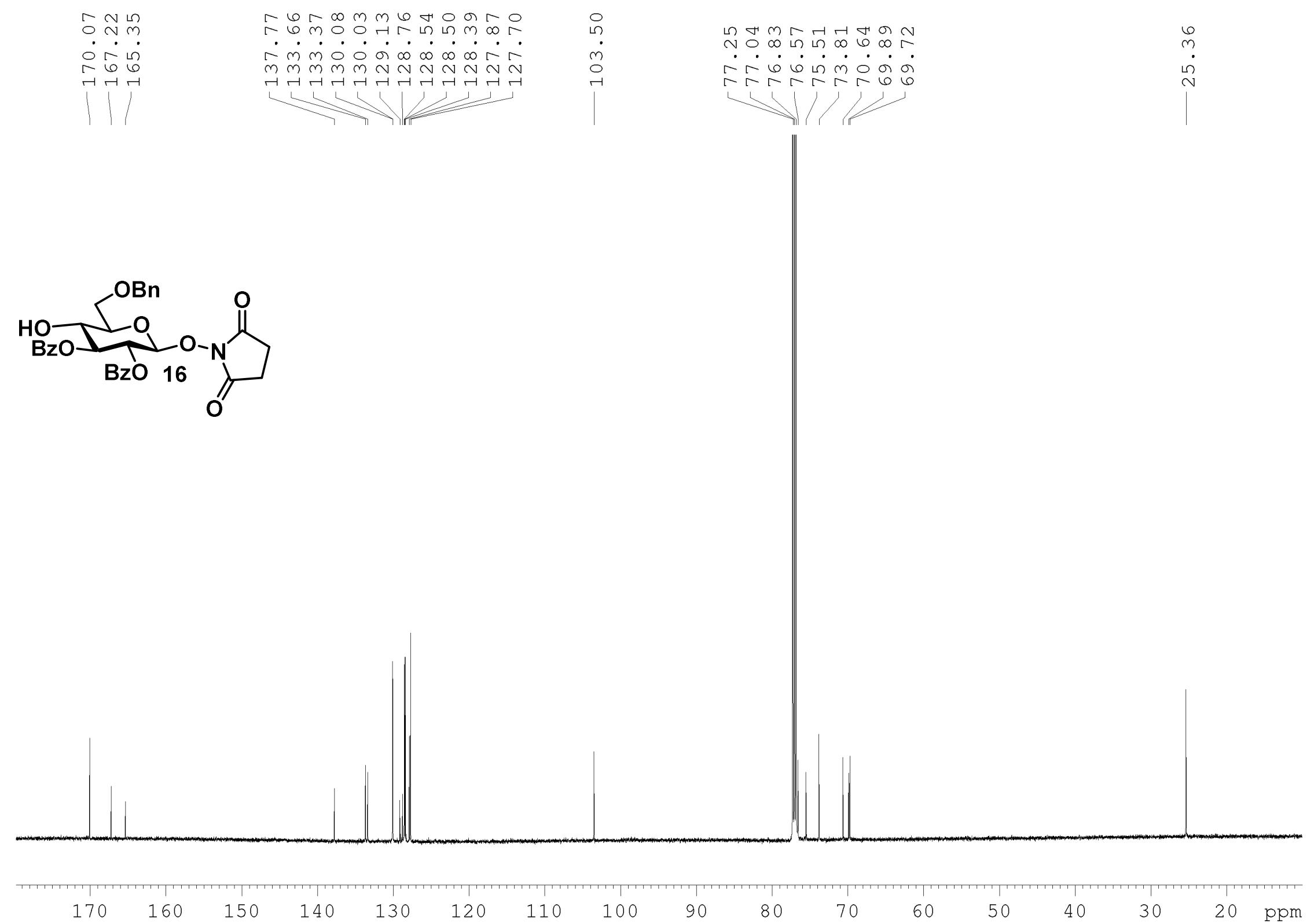


$\underline{\text { DEPT NMR; } 150 \mathrm{MHz} ; \mathrm{CDCl}_{3}}$

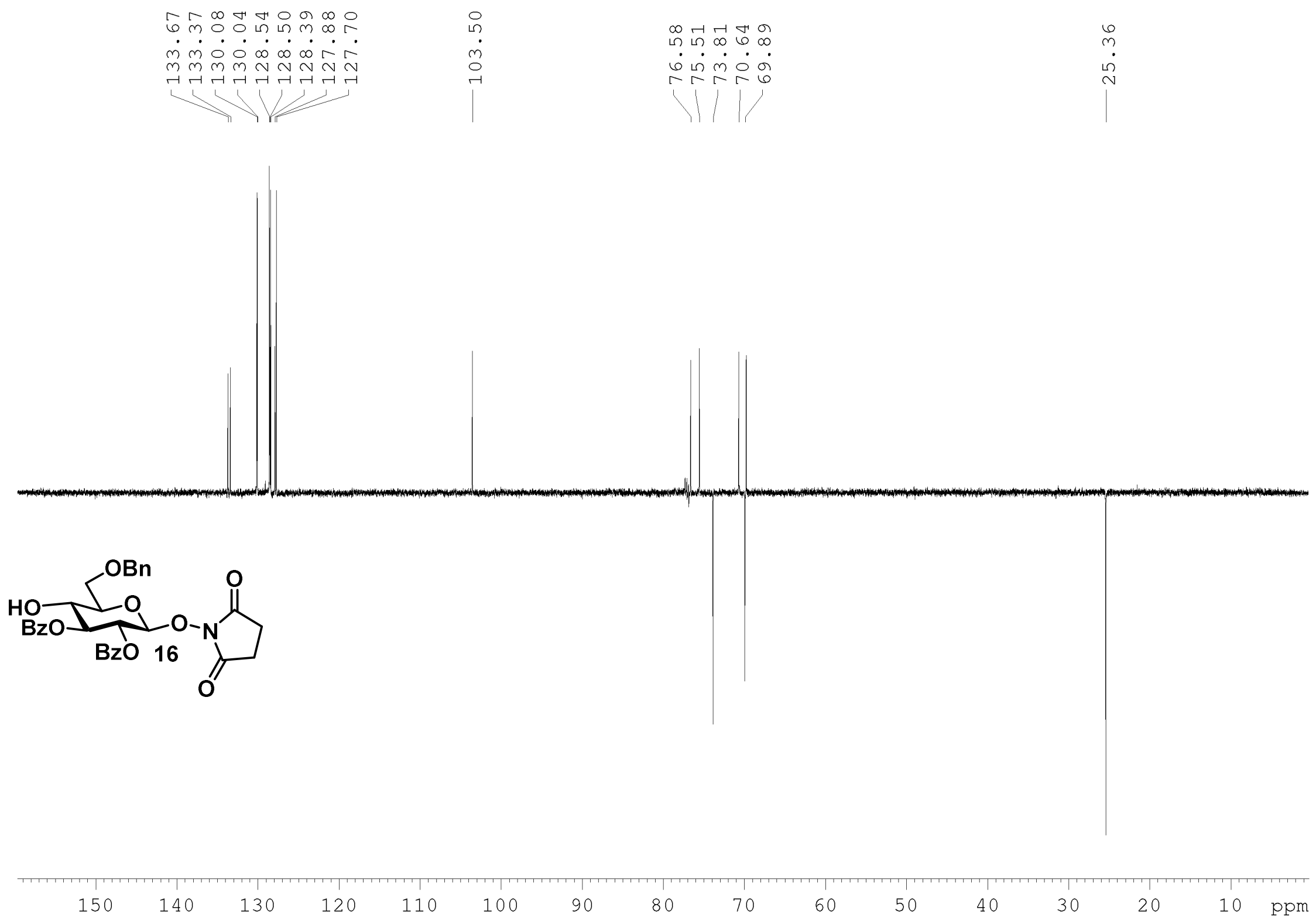




\section{COSY NMR; $600 \mathrm{MHz} ; \mathrm{CDCl}_{3}$}

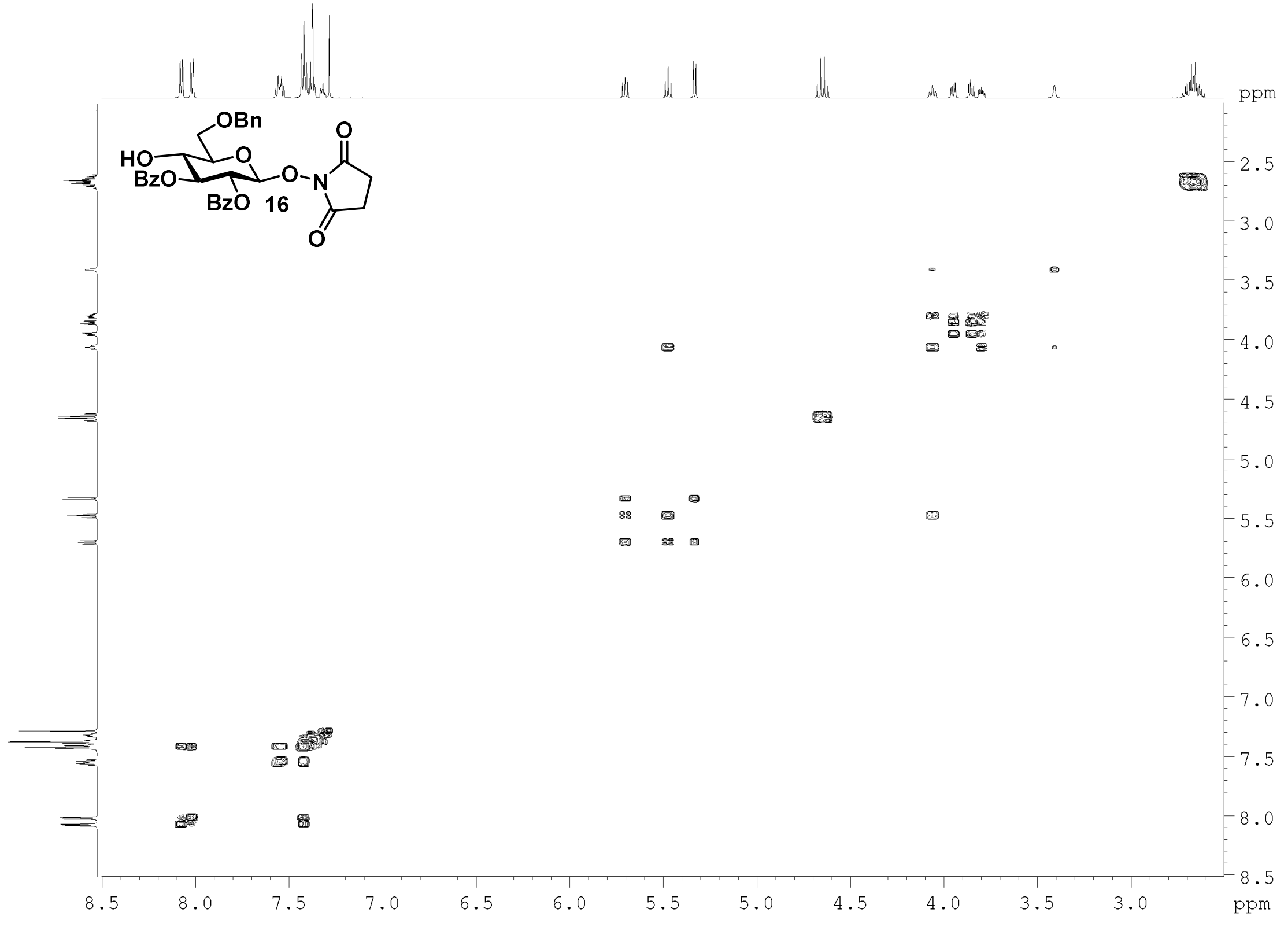




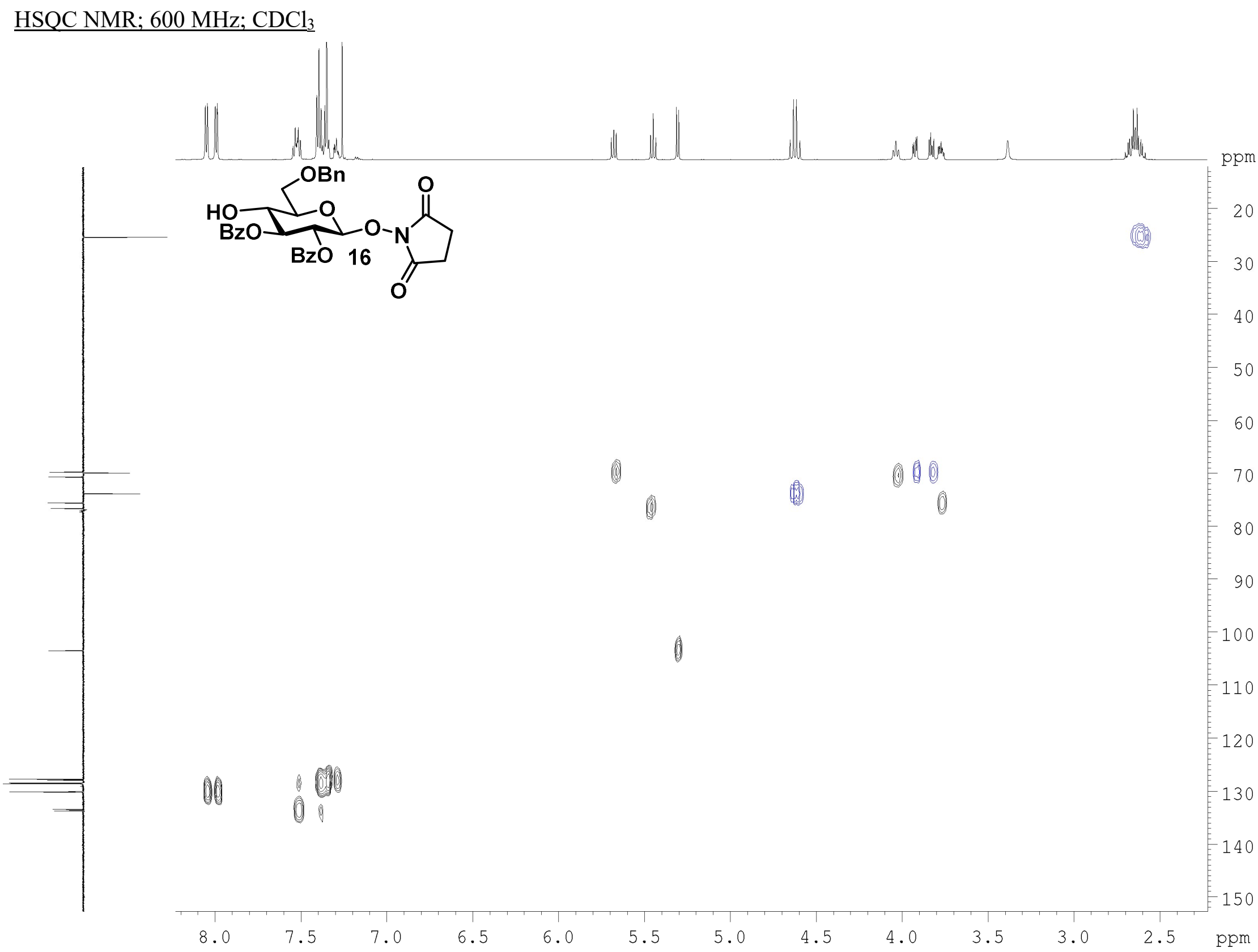




\section{$\underline{\mathrm{HMBC} N M R ; 600 \mathrm{MHz} ; \mathrm{CDCl}_{3}}$}

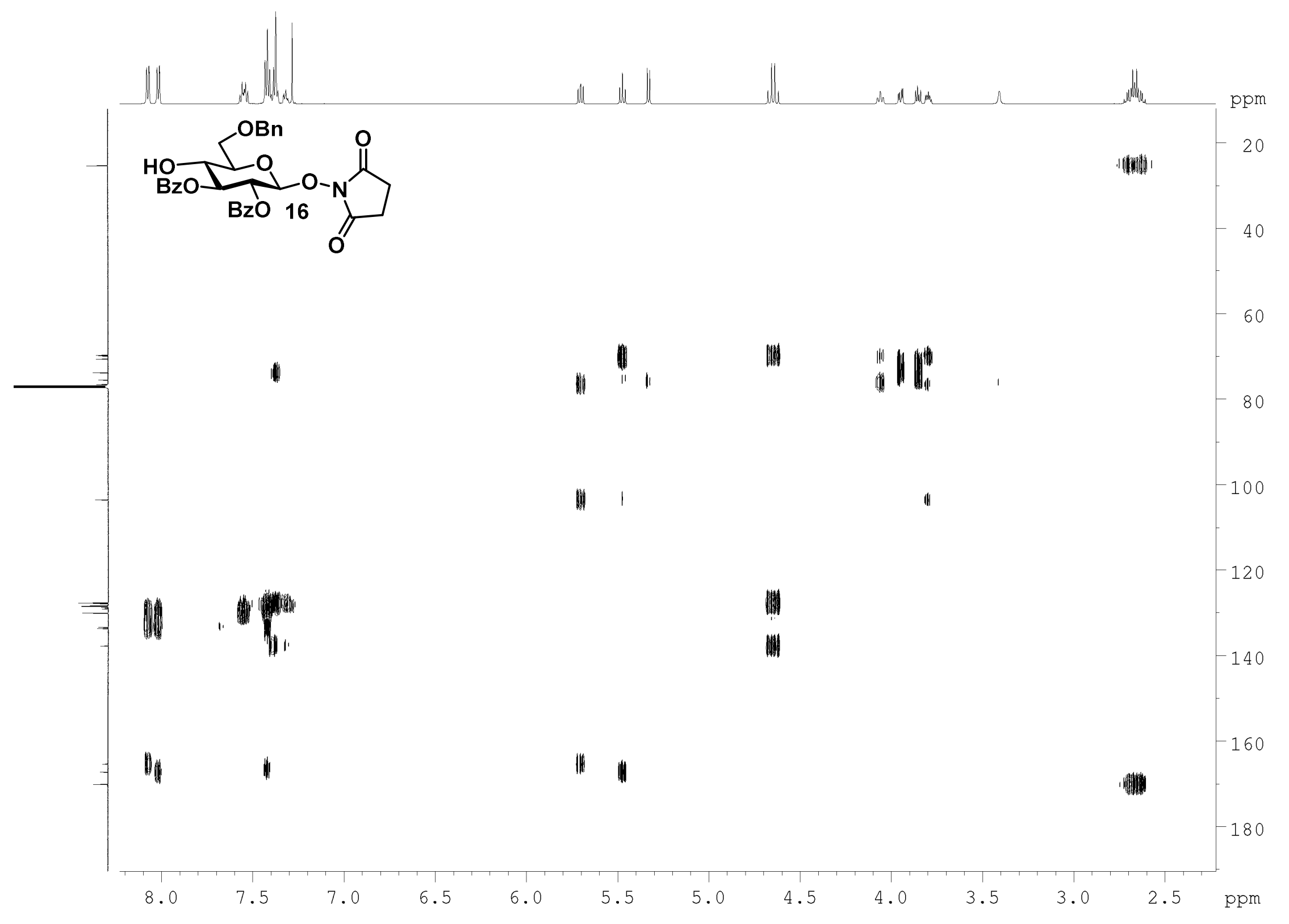




\section{$\underline{{ }^{1} \mathrm{H} \mathrm{NMR} ; 600 \mathrm{MHz} ; \mathrm{CDCl}_{3}}$}

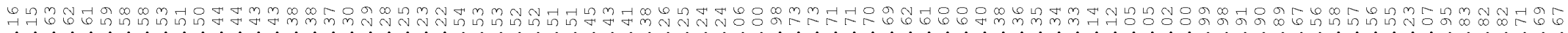
(1)

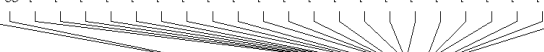
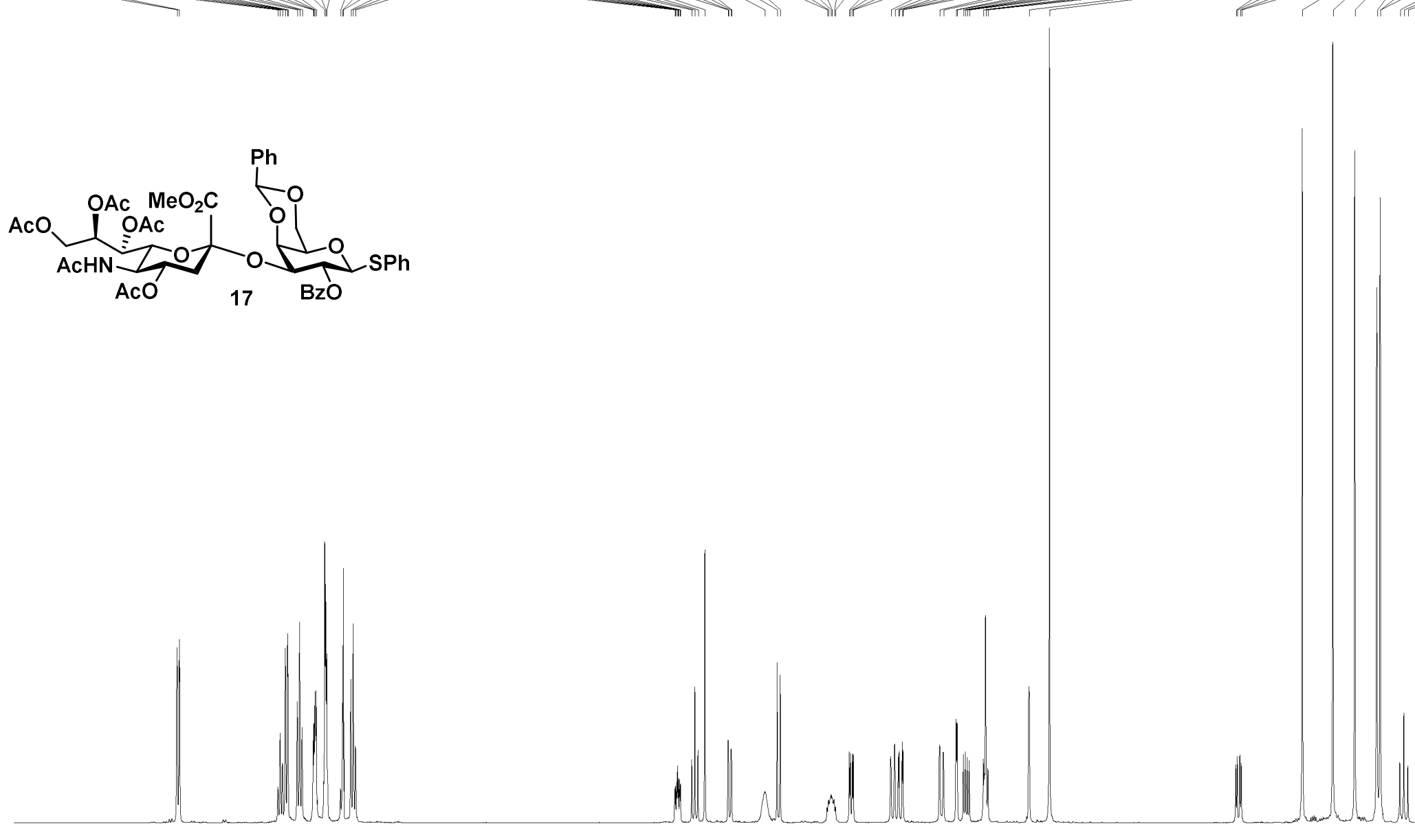

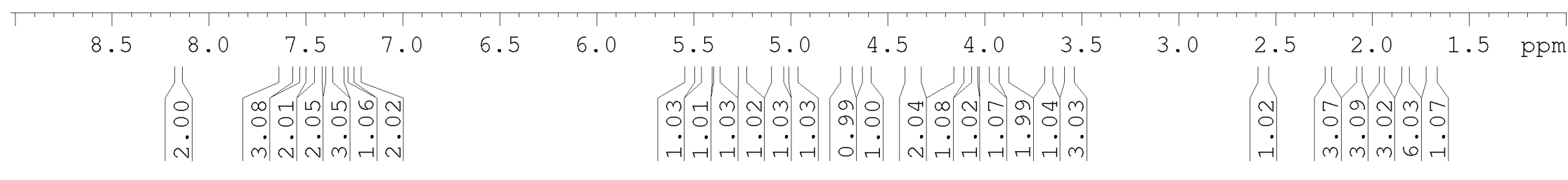


${ }^{13} \mathrm{C} \mathrm{NMR} ; 150 \mathrm{MHz} ; \mathrm{CDCl}_{3}$

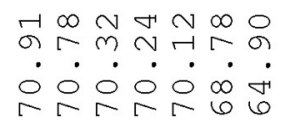

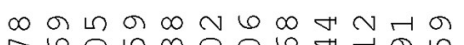

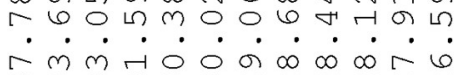

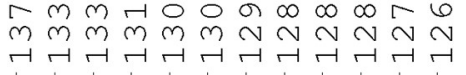
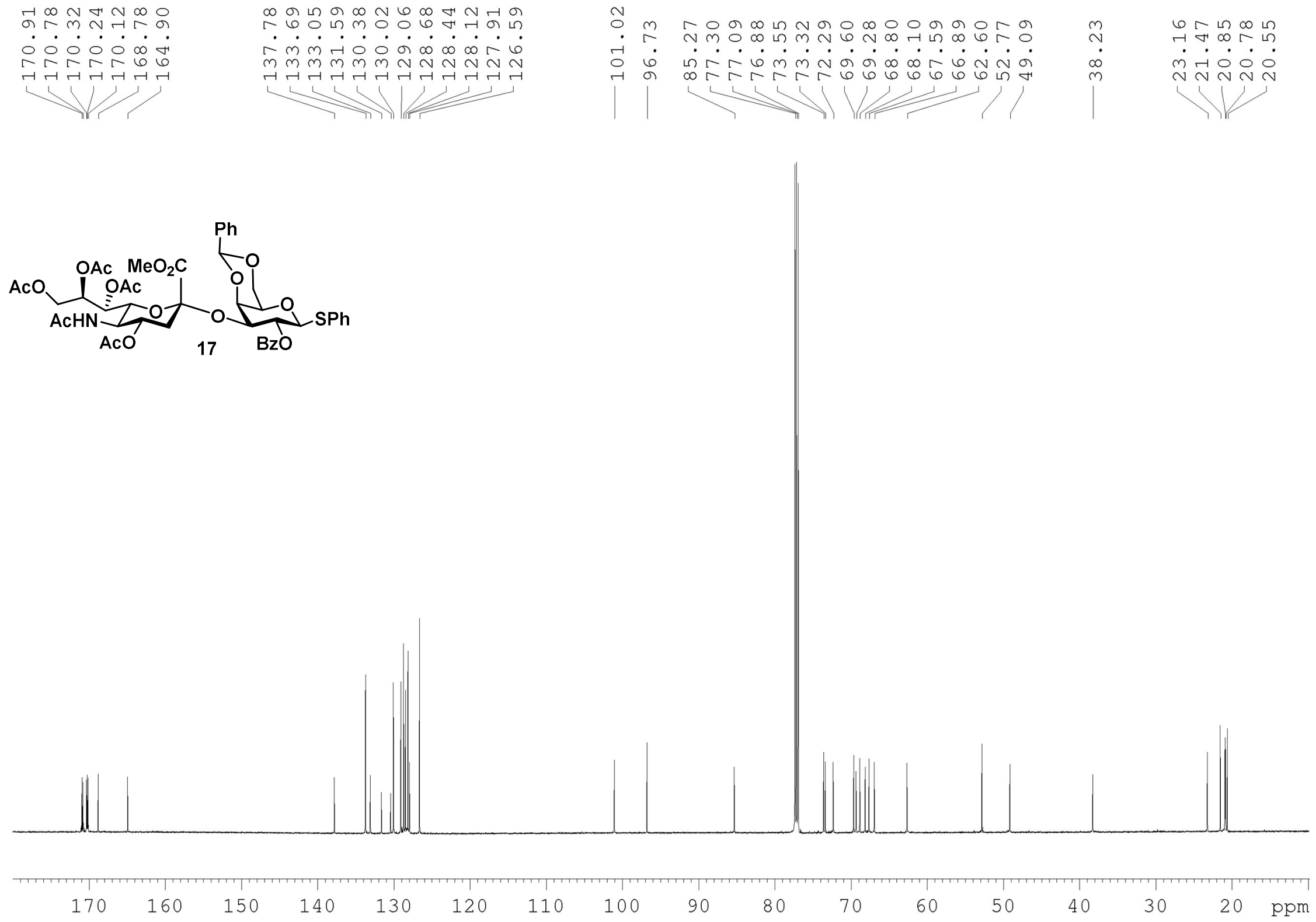


\section{$\underline{\text { DEPT NMR; } 150 \mathrm{MHz} ; \mathrm{CDCl}_{3}}$}

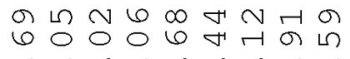

$\dot{m} \dot{m} \dot{0} \dot{\sigma} \dot{\infty} \dot{\infty} \dot{\sim} \dot{0}$

mmm $N \underset{H}{N} N \underset{H}{N} \mathbb{H}$

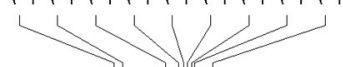

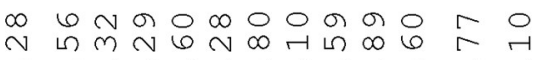
. . . . . . . . . . .

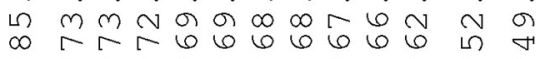

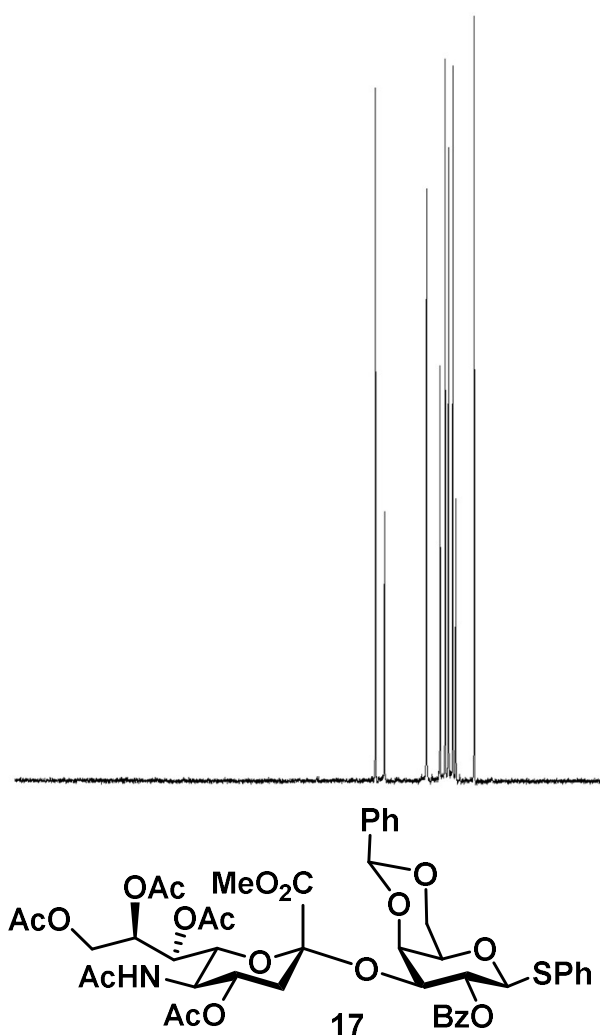

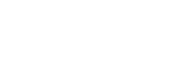

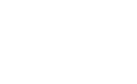




\section{$\underline{\text { COSY NMR; } 600 \mathrm{MHz} ; \mathrm{CDCl}_{3}}$}

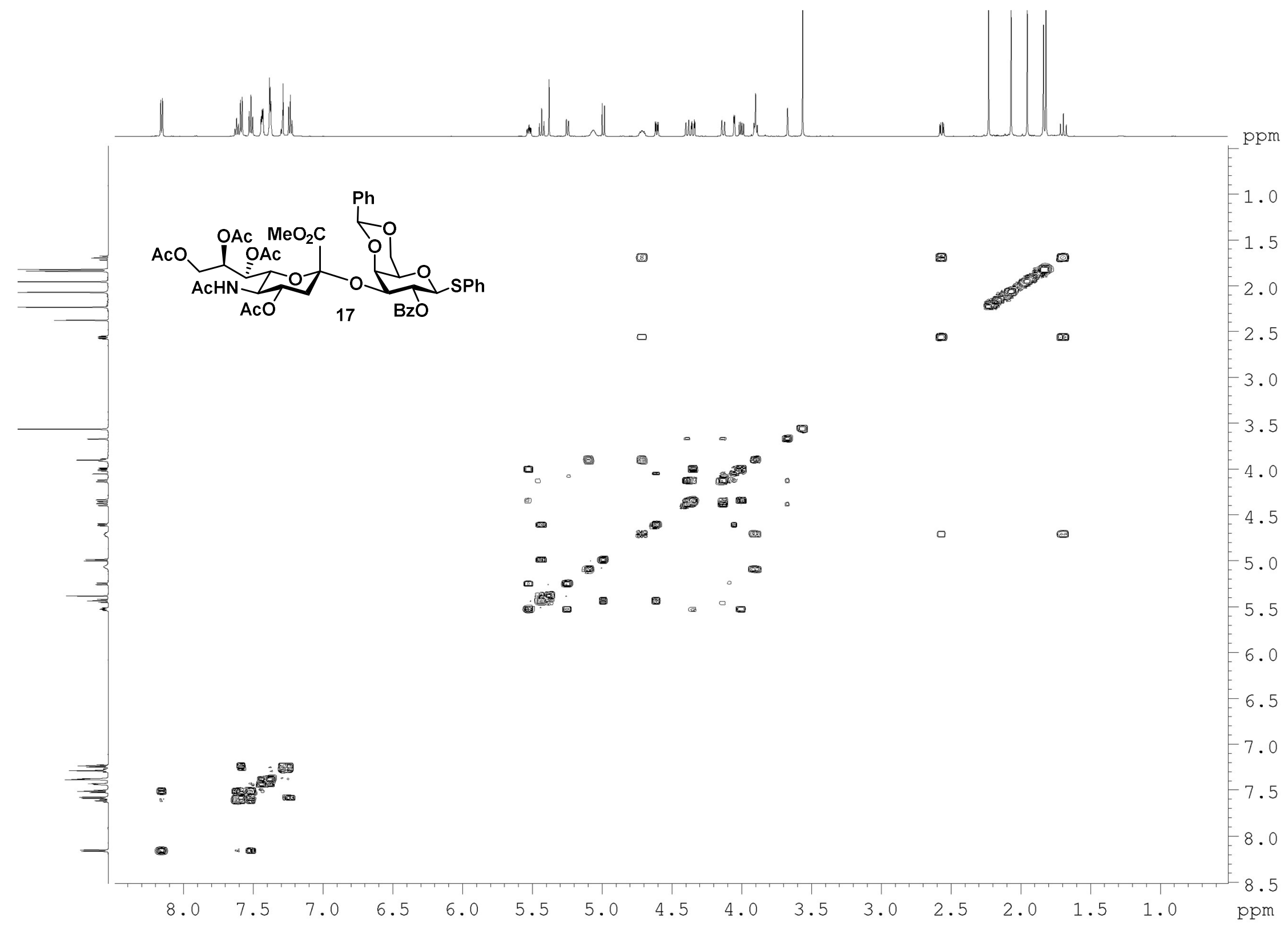




\section{$\underline{\mathrm{HSQC} N M R ; 600 \mathrm{MHz} ; \mathrm{CDCl}_{3}}$}

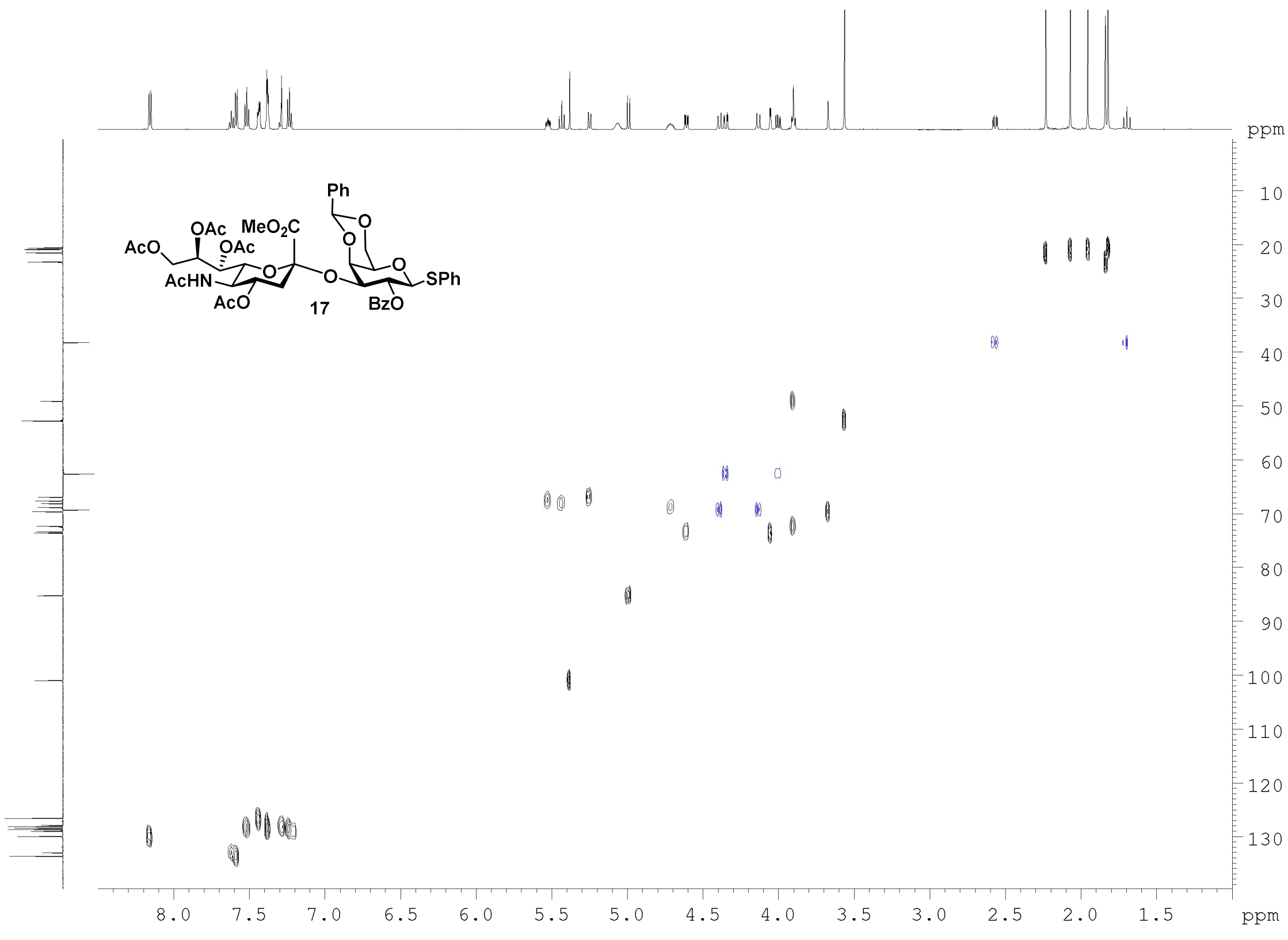




\section{$\underline{\mathrm{HMBC} N M R ; 600 \mathrm{MHz} ; \mathrm{CDCl}_{3}}$}

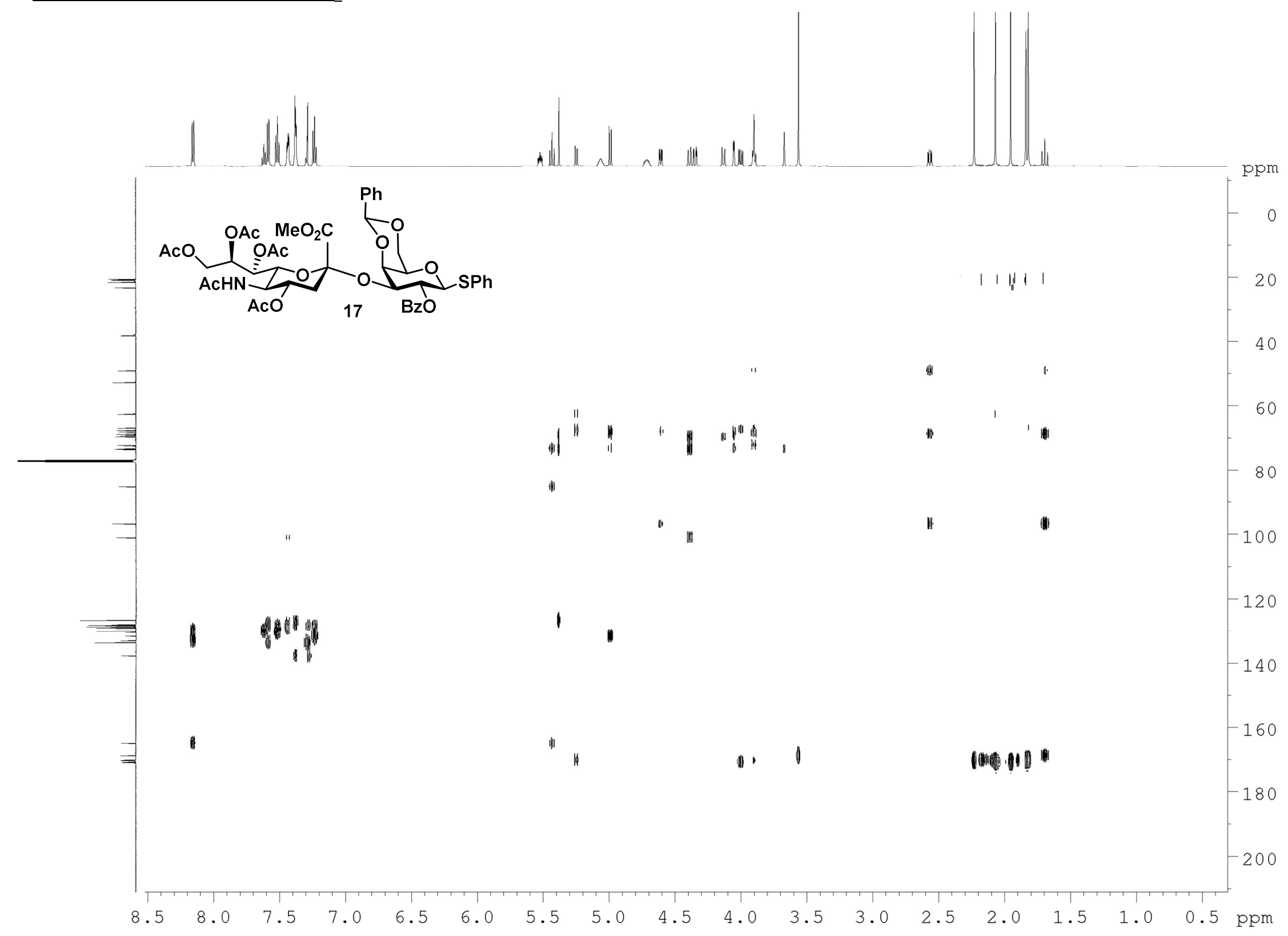




\section{$\underline{{ }^{1} \mathrm{H} N M R ; 600 \mathrm{MHz} ; \mathrm{CDCl}_{3}}$}

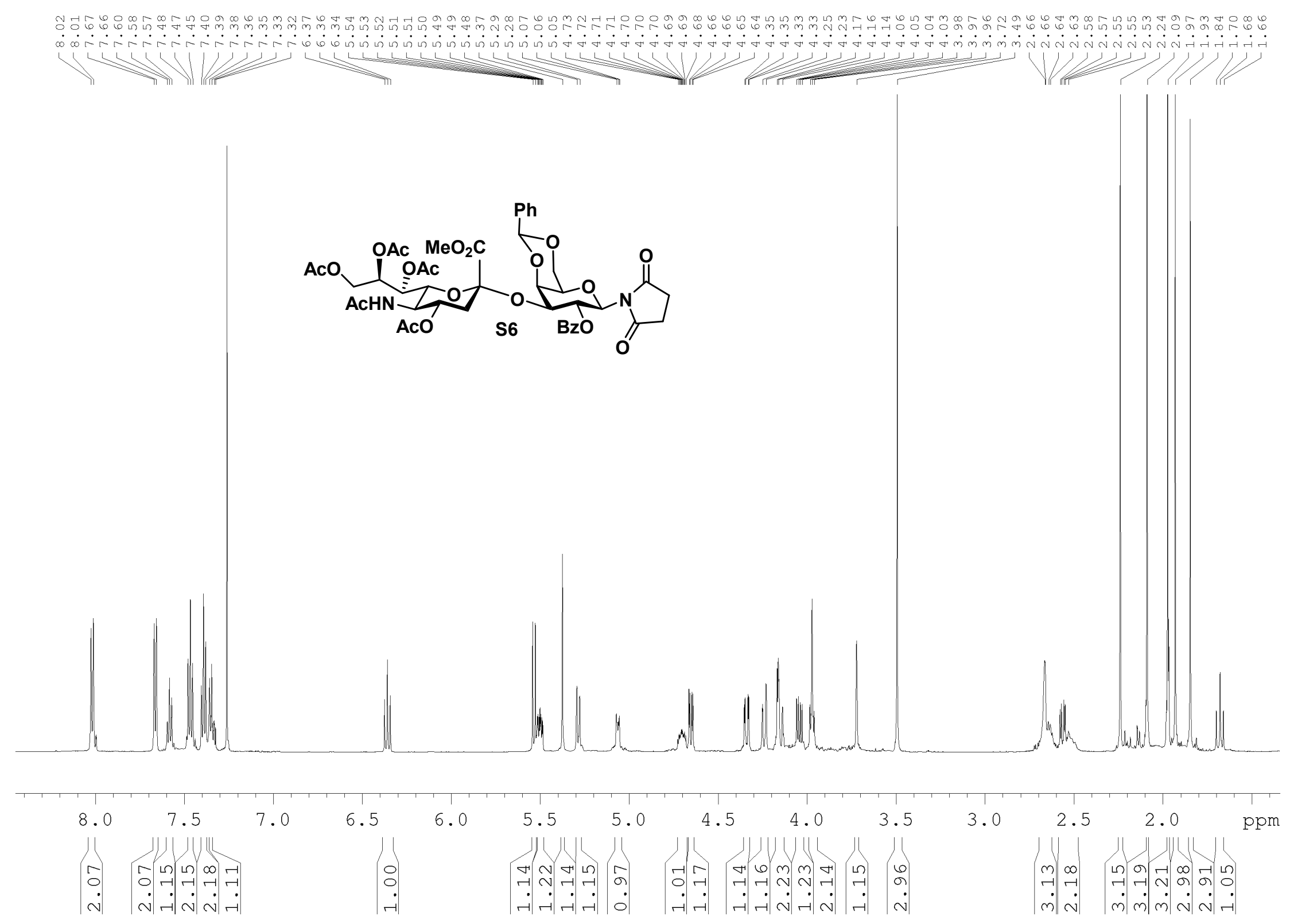


${ }^{13} \mathrm{C} \mathrm{NMR} ; 150 \mathrm{MHz} ; \mathrm{CDCl}_{3}$

$\begin{array}{lllll}4 & 0\end{array}$
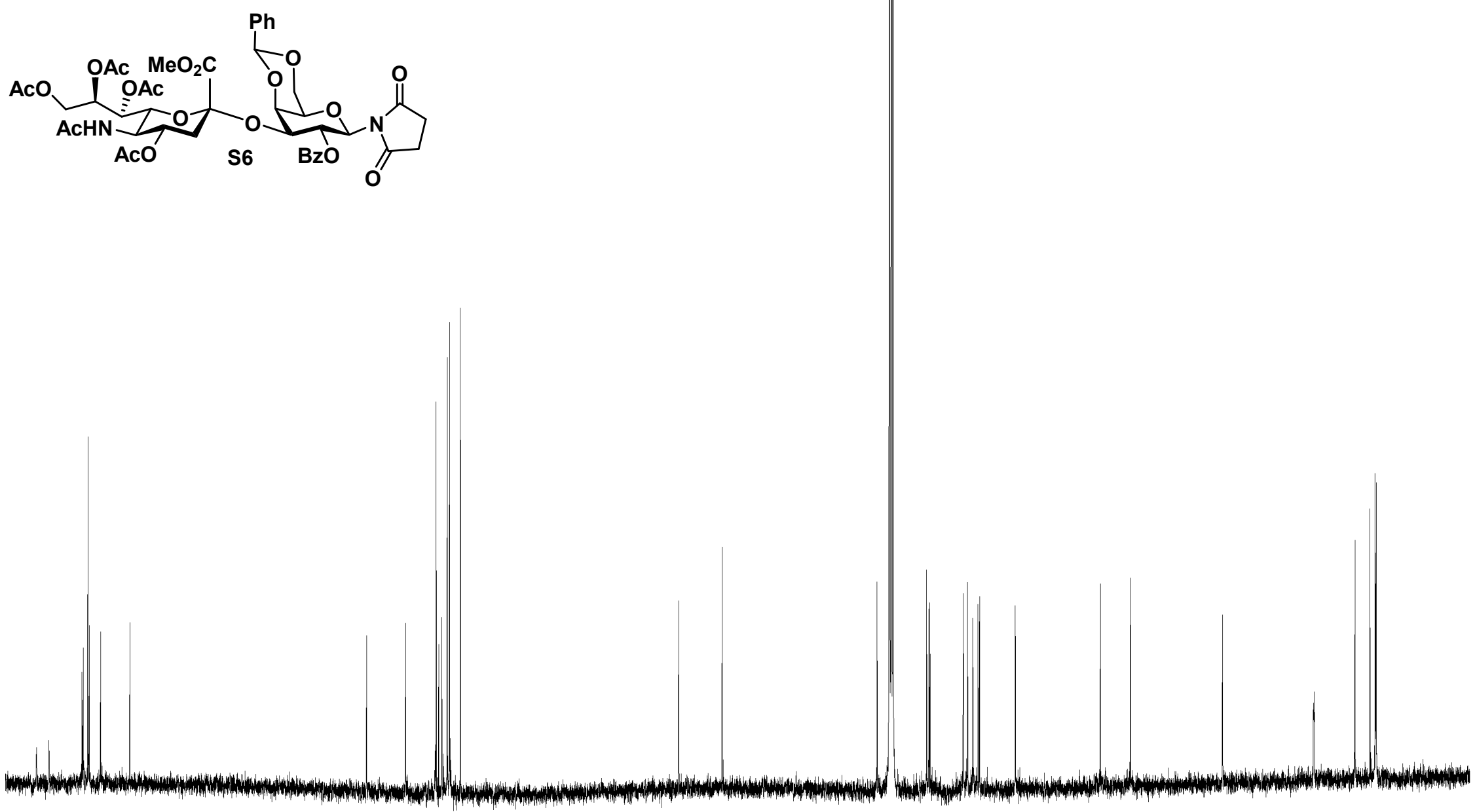

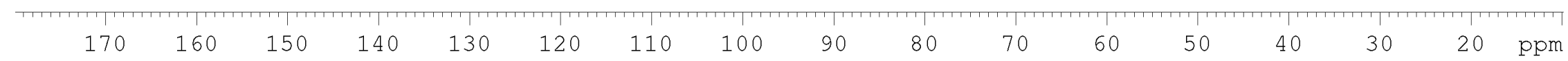




\section{DEPT NMR; $150 \mathrm{MHz} ; \mathrm{CDCl}_{3}$}

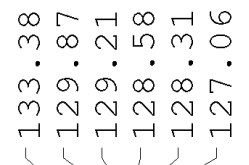

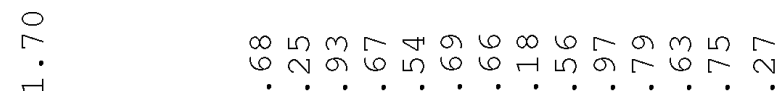
$\dot{\infty} \dot{0} \dot{\infty} \dot{\infty} \dot{0} \dot{0} \dot{0} \dot{\sigma}$

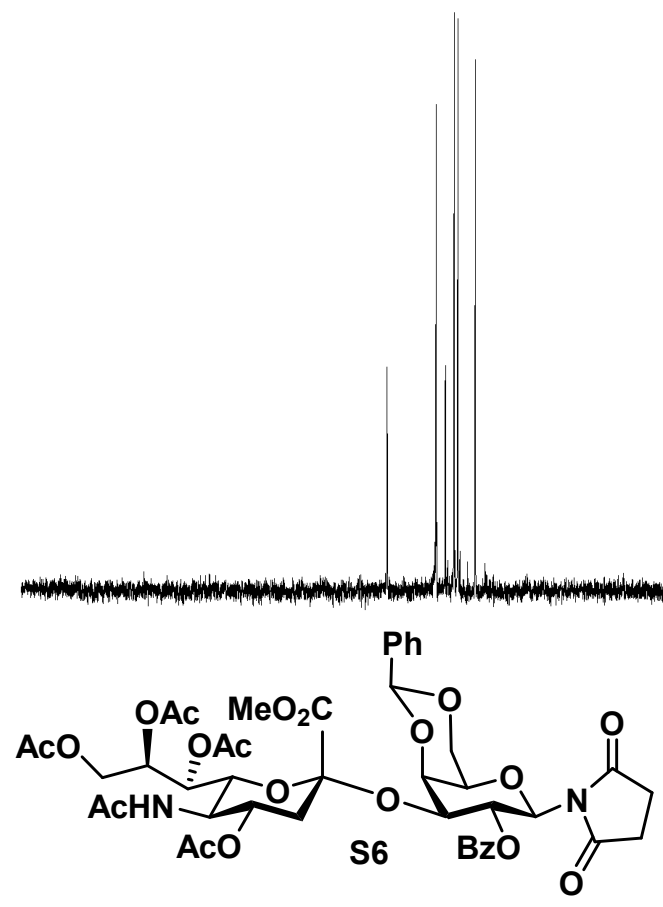

$\stackrel{\infty}{\sim}$

th

m $\quad$ Nㅔ융ำ

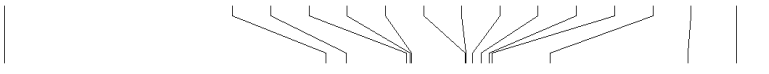

$\rightarrow 12$

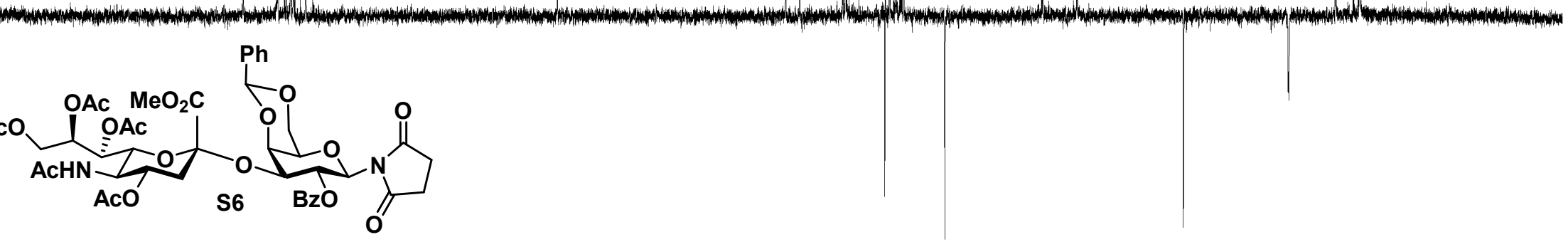

70

60

50

40

30

20

$10 \mathrm{ppm}$ 


\section{$\underline{\text { COSY NMR; } 600 \mathrm{MHz} ; \mathrm{CDCl}_{3}}$}

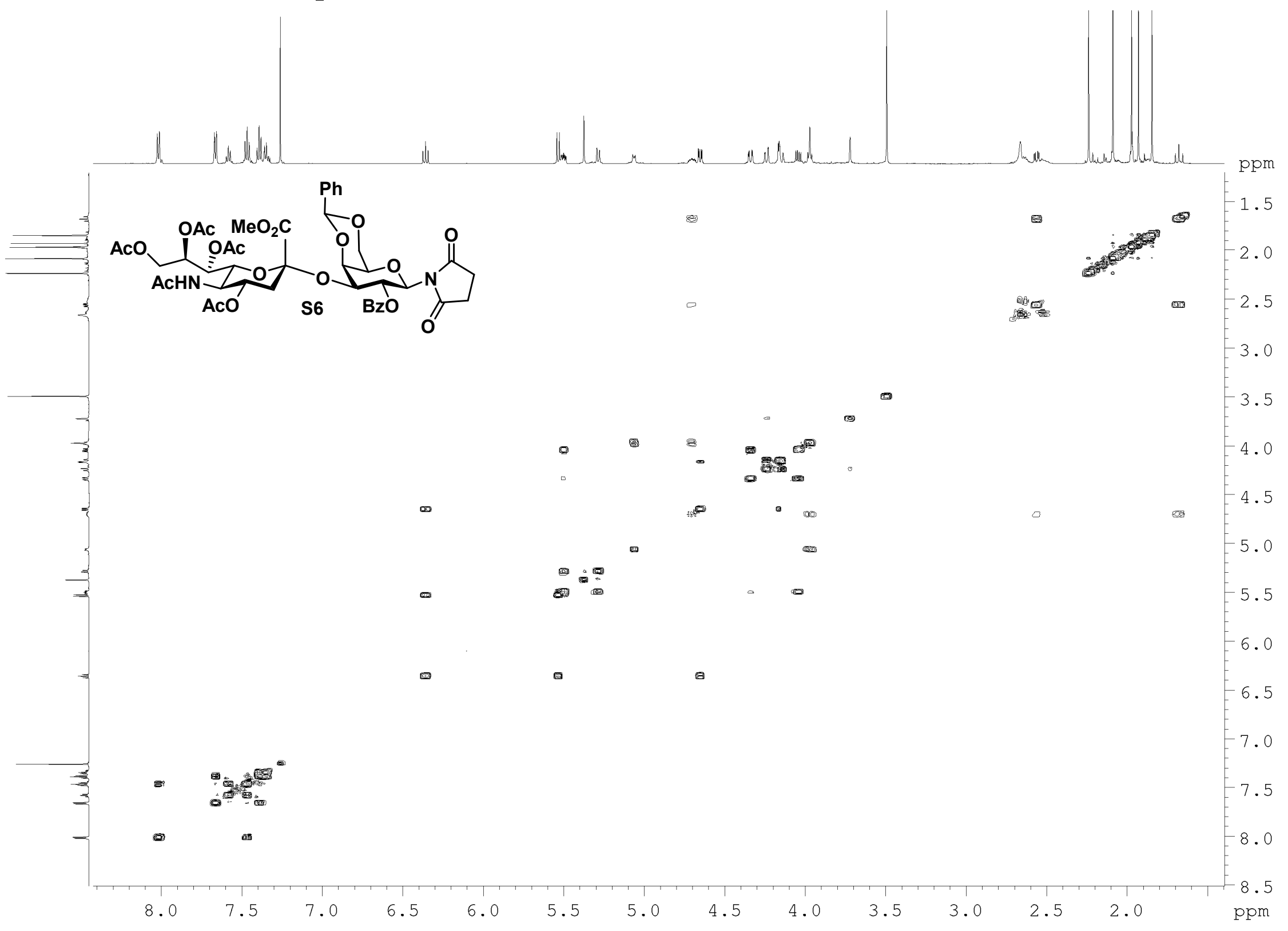


$\underline{\mathrm{HSQC} N M R ; 600 \mathrm{MHz} ; \mathrm{CDCl}_{3}}$

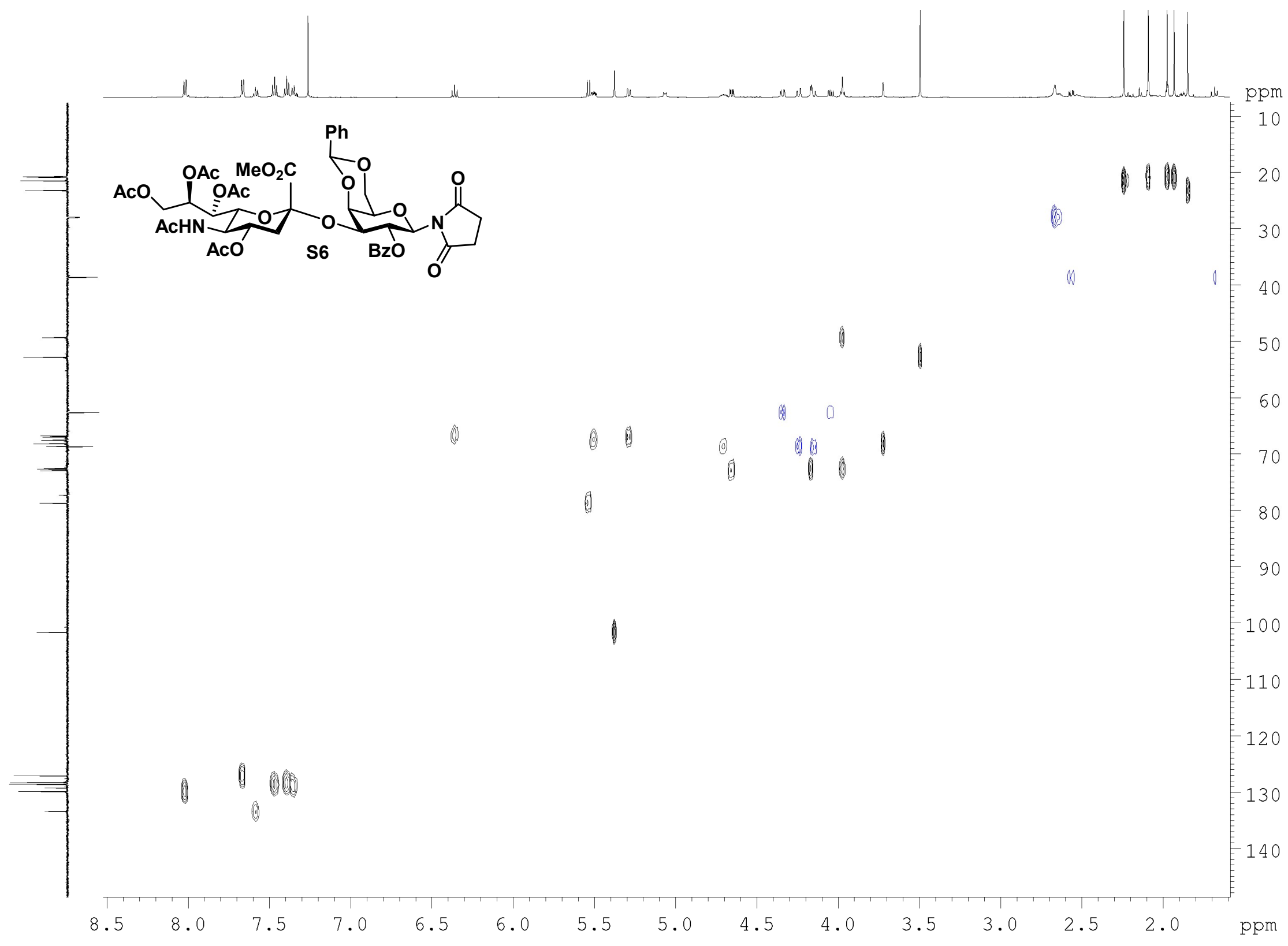




\section{$\underline{\mathrm{HMBC} N M R ; 600 \mathrm{MHz} ; \mathrm{CDCl}_{3}}$}

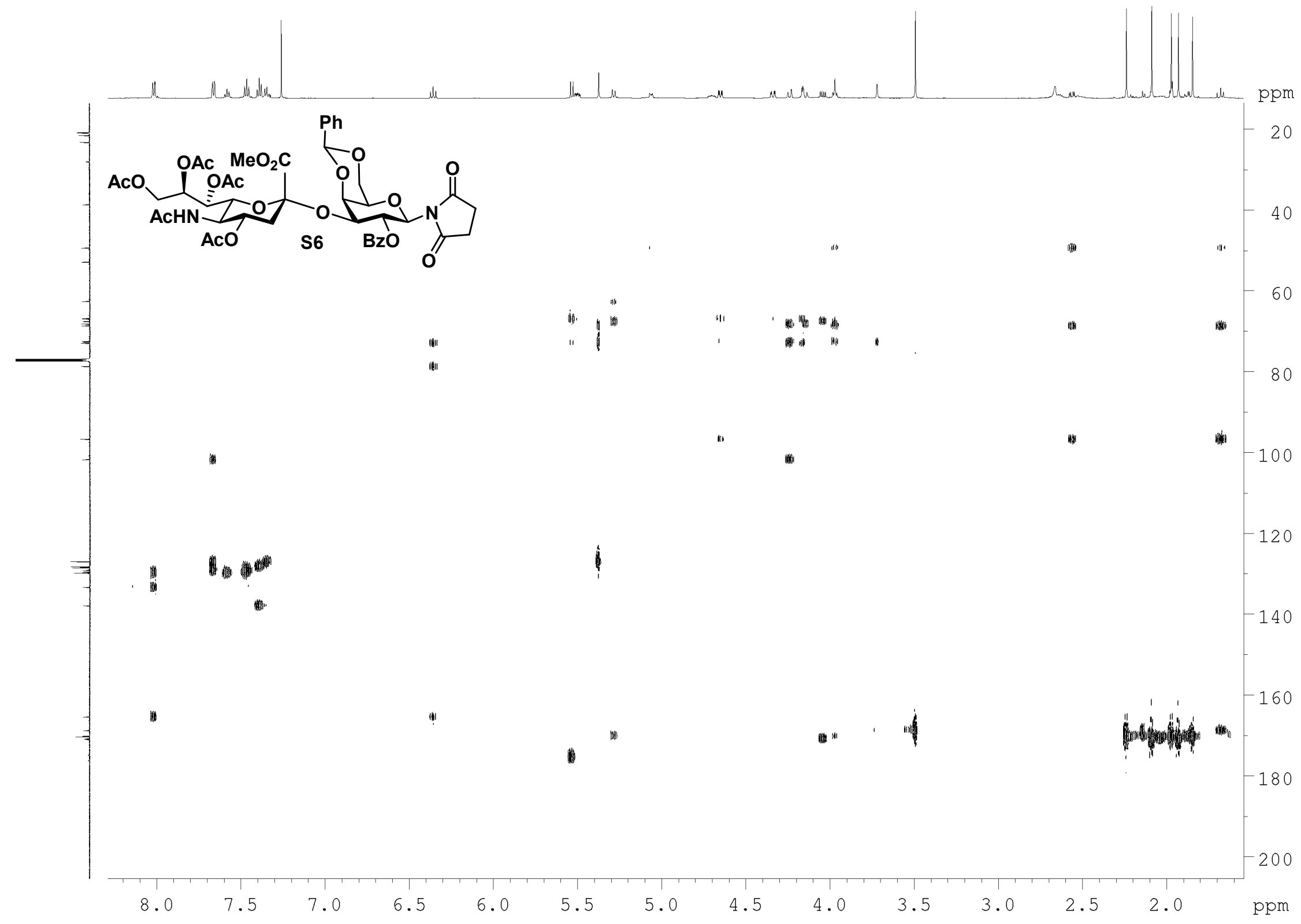




\section{${ }^{1} \mathrm{H} \mathrm{NMR} ; 600 \mathrm{MHz} ; \mathrm{CDCl}_{3}$}

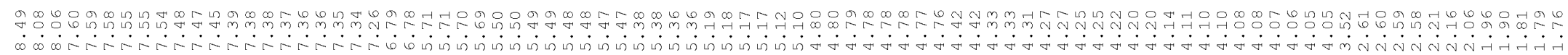

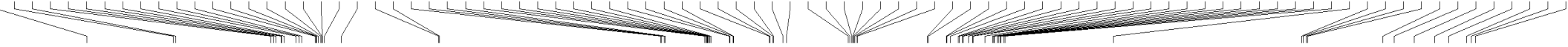

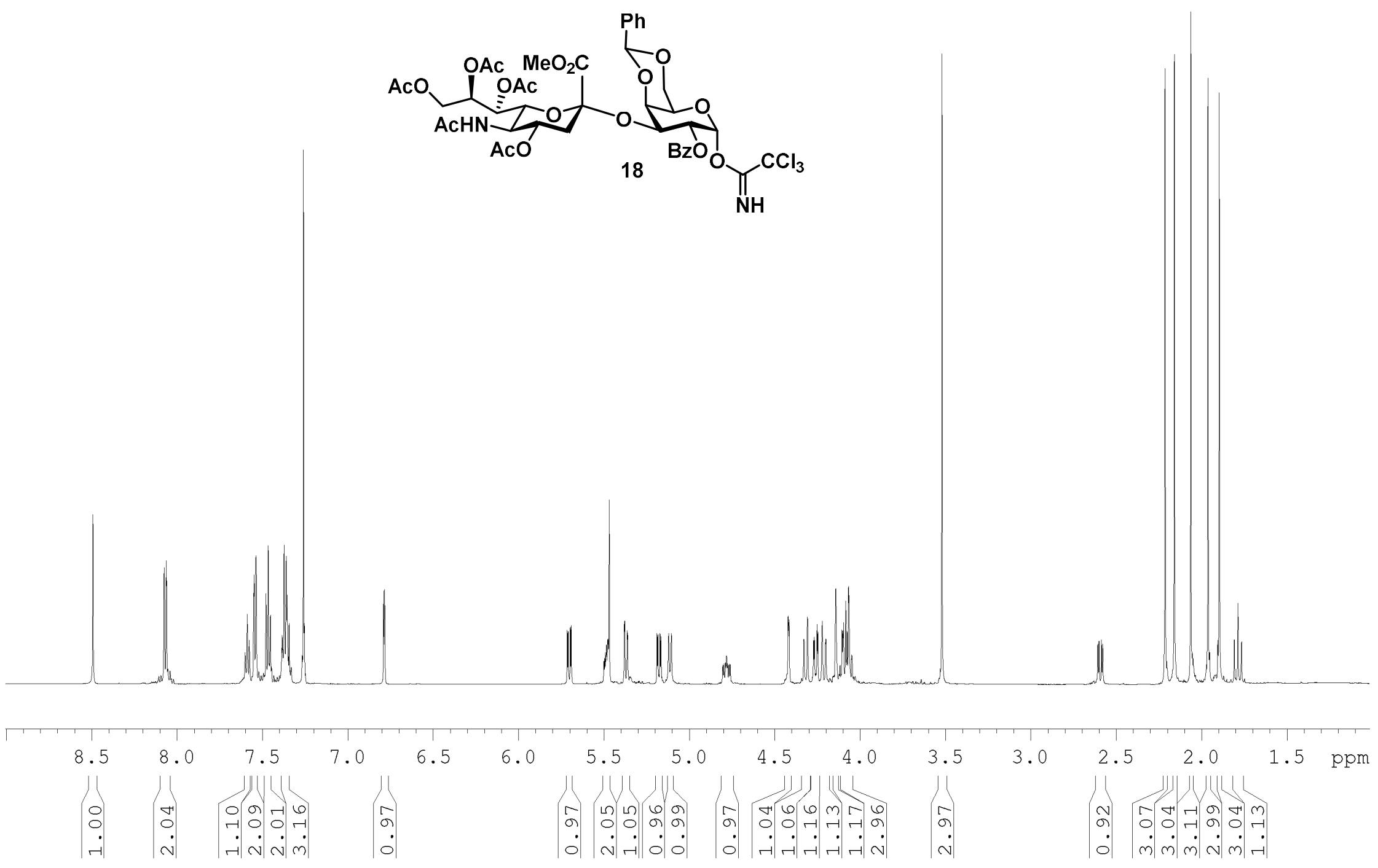




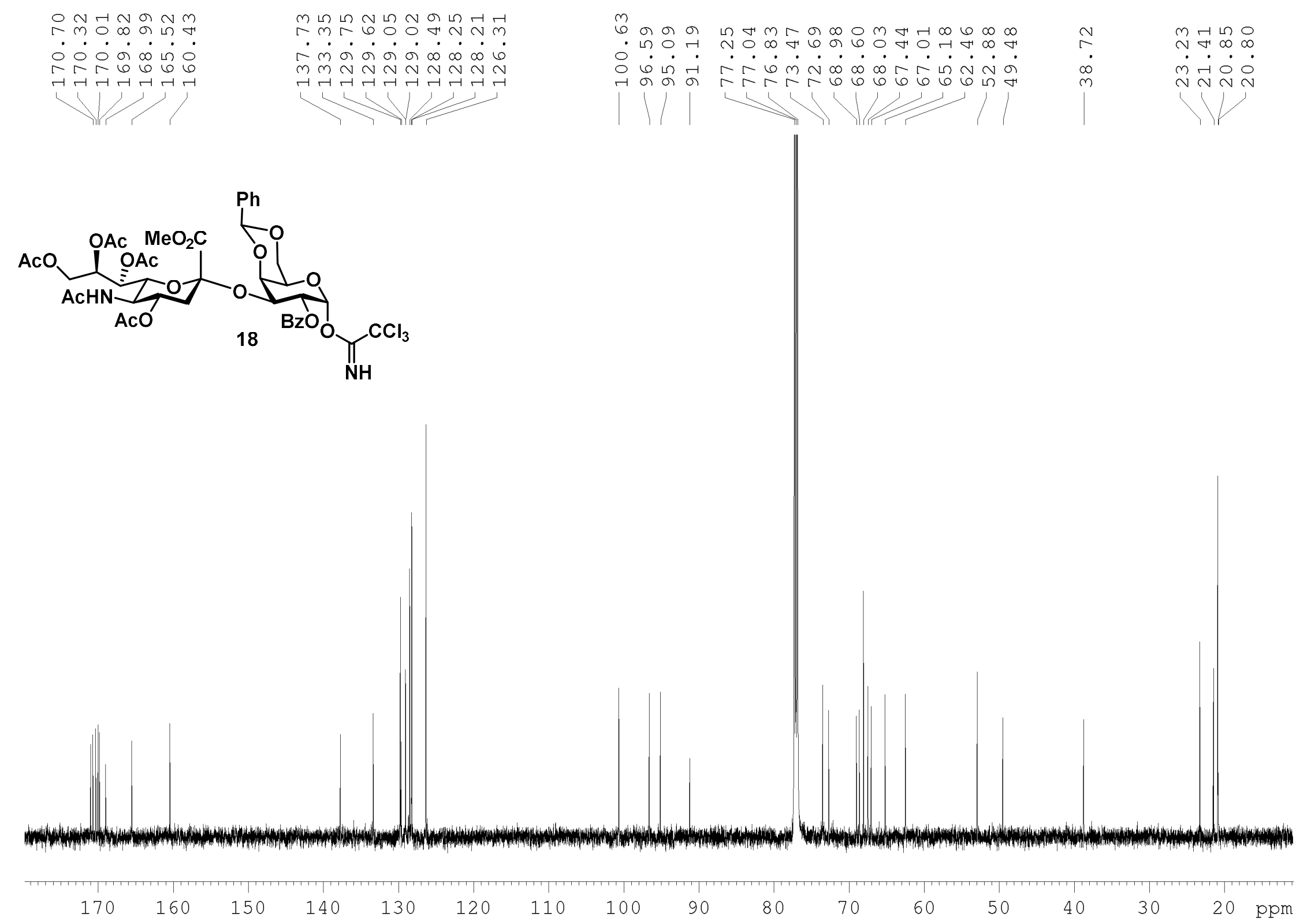




\section{$\underline{\text { DEPT NMR; } 150 \mathrm{MHz} ; \mathrm{CDCl}_{3}}$}

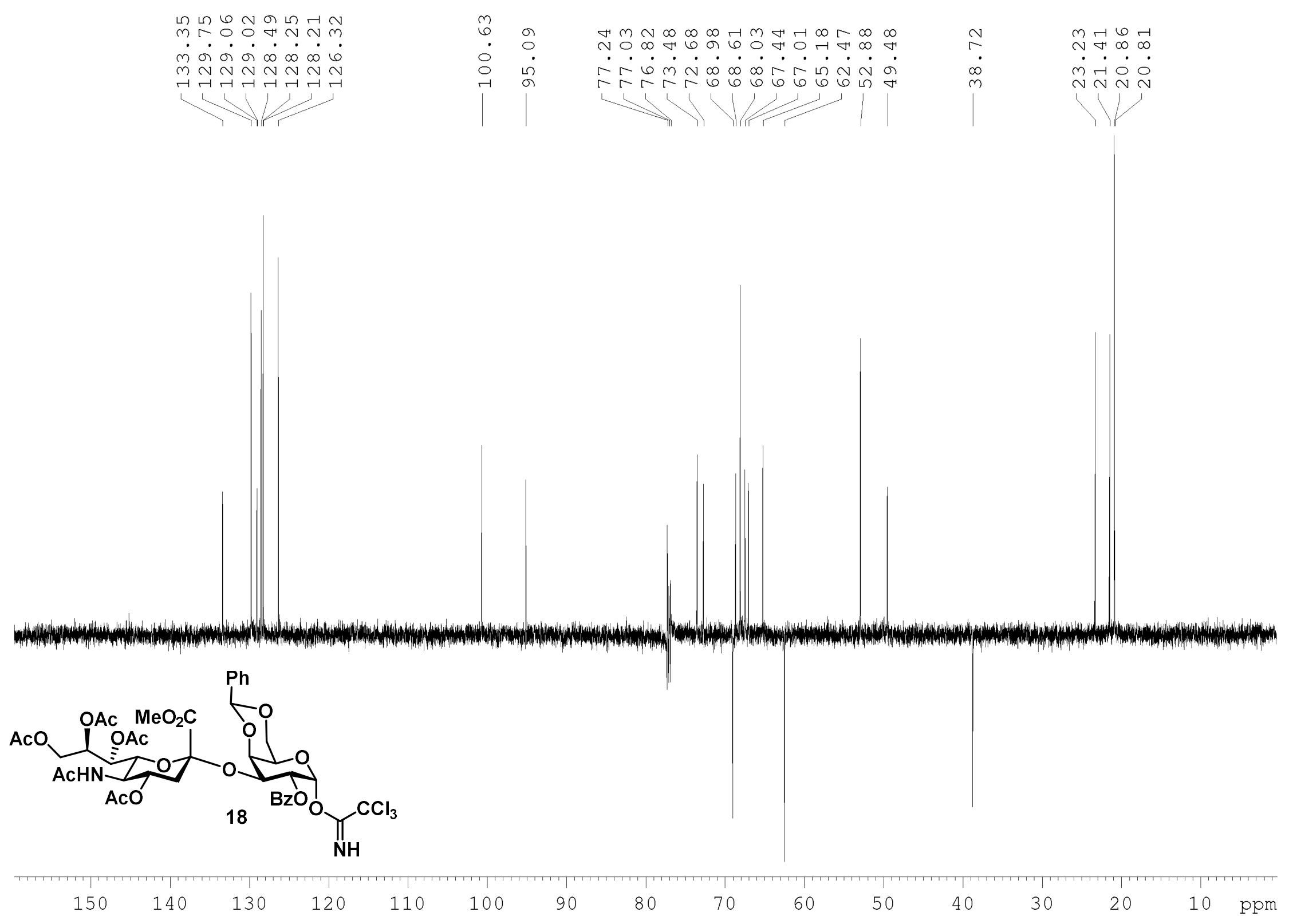


$\underline{\text { COSY NMR; } 600 \mathrm{MHz} ; \mathrm{CDCl}_{3}}$

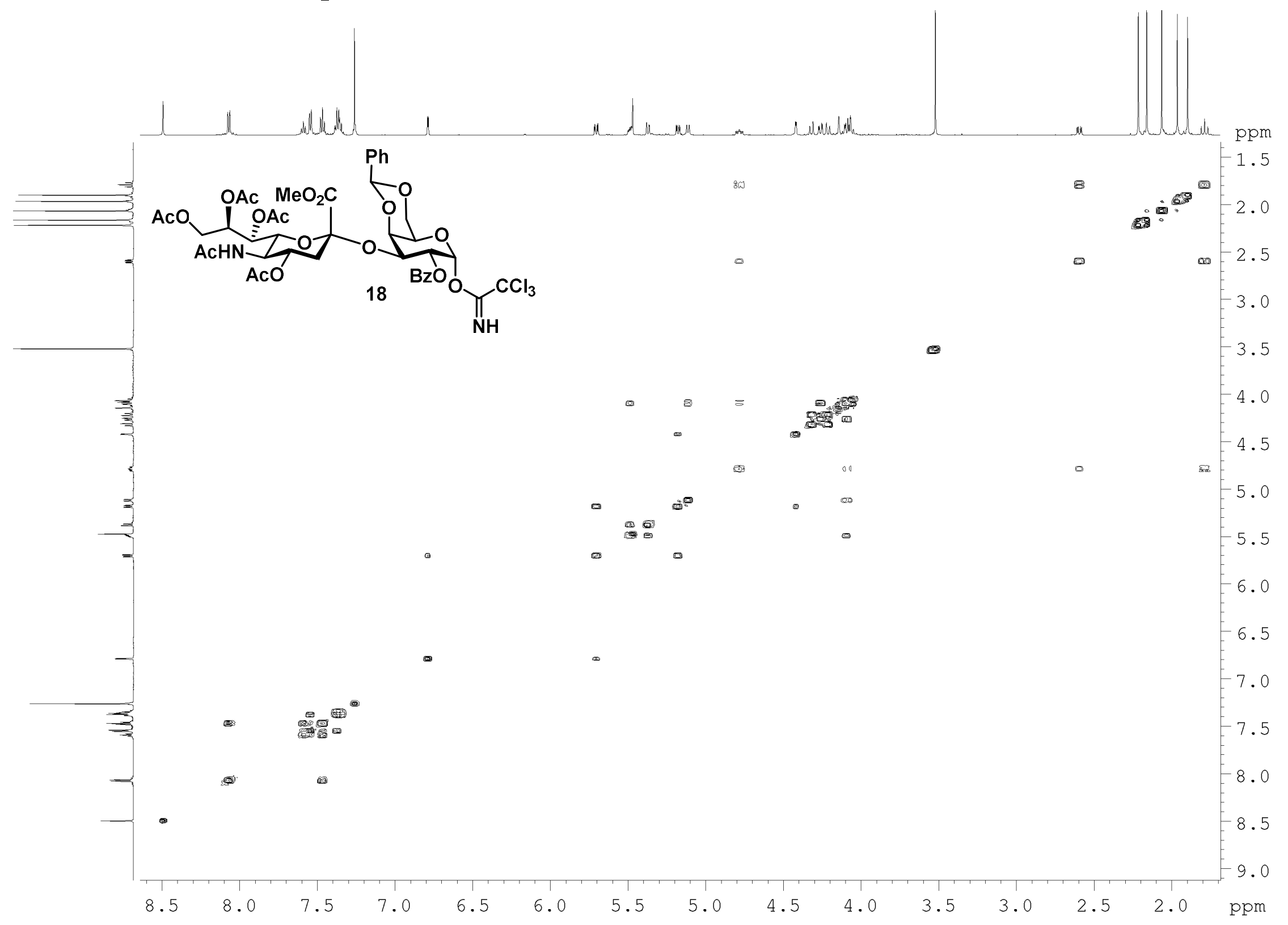




\section{$\underline{{ }^{1} \mathrm{H} N M R ; 600 \mathrm{MHz} ; \mathrm{CDCl}_{3}}$}

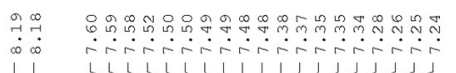
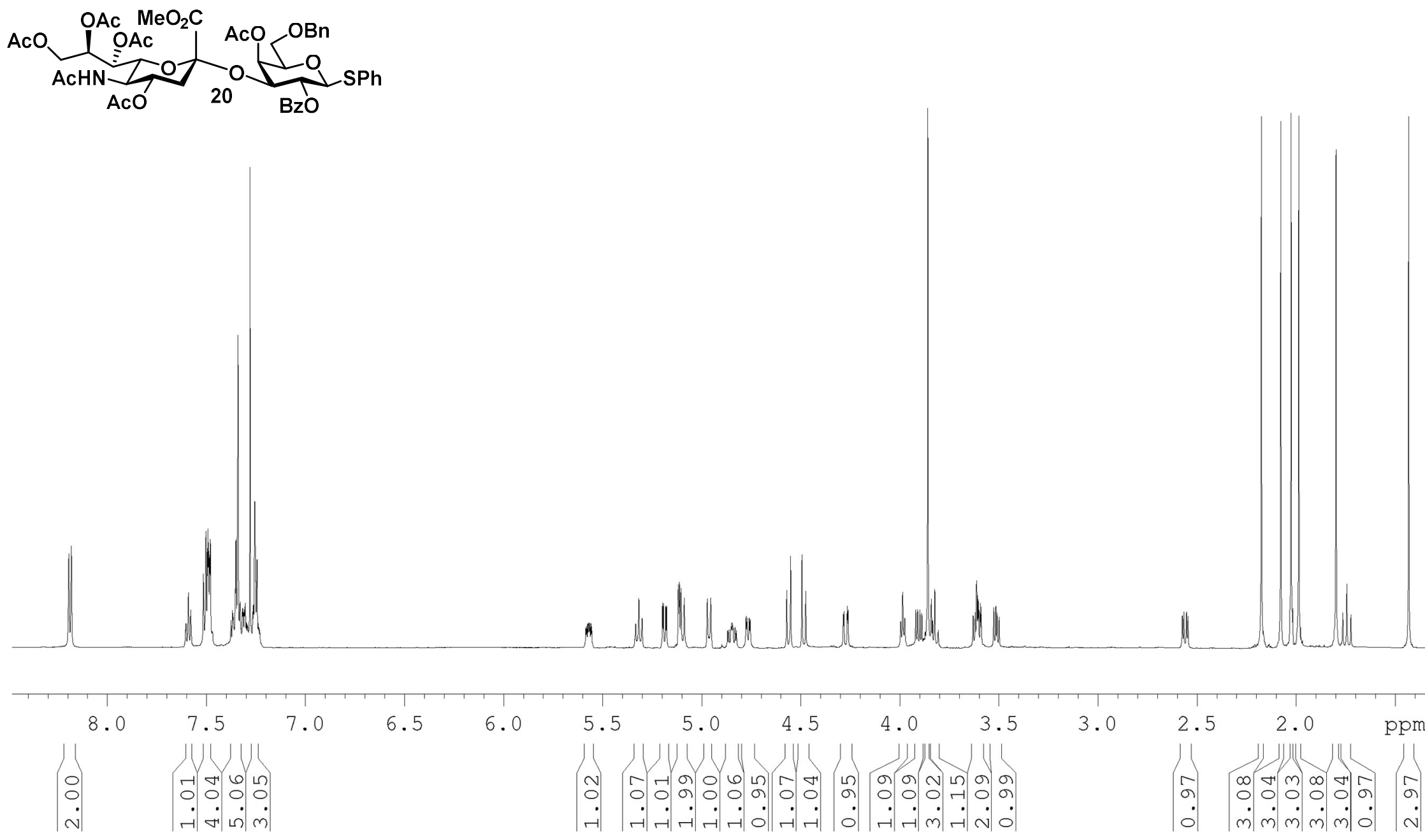

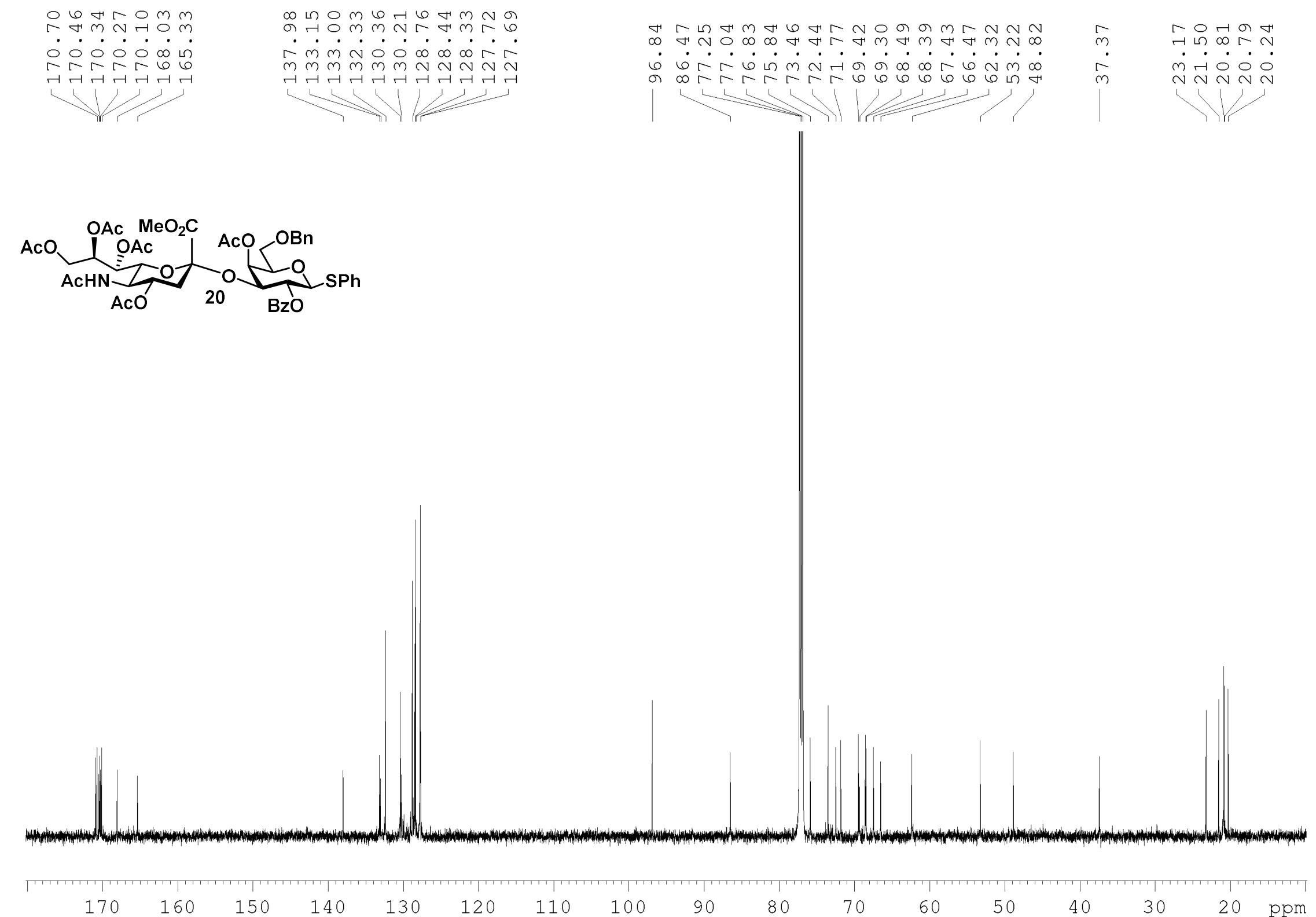


\section{$\underline{\text { DEPT NMR; } 150 \mathrm{MHz} ; \mathrm{CDCl}_{3}}$}

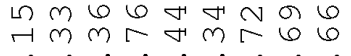

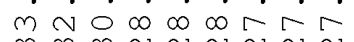

$m m m \sim N \sim N N$

只鸟

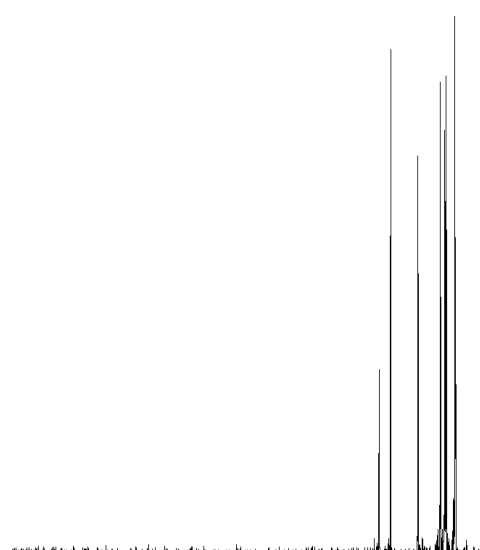

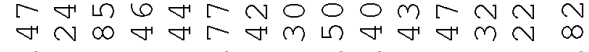

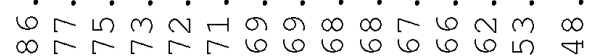$$
\text { का }
$$

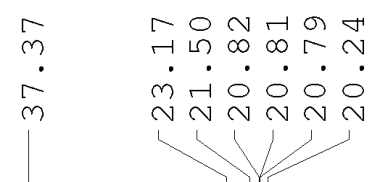

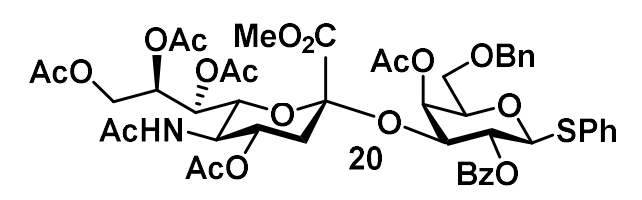

\begin{tabular}{|c|c|c|c|c|c|c|c|c|c|c|c|c|c|c|}
\hline 150 & 140 & 130 & 120 & 110 & 100 & 90 & 80 & 70 & 60 & 50 & 40 & 30 & 20 & ppm \\
\hline
\end{tabular}




\section{$\underline{\text { COSY NMR; } 600 \mathrm{MHz} ; \mathrm{CDCl}_{3}}$}

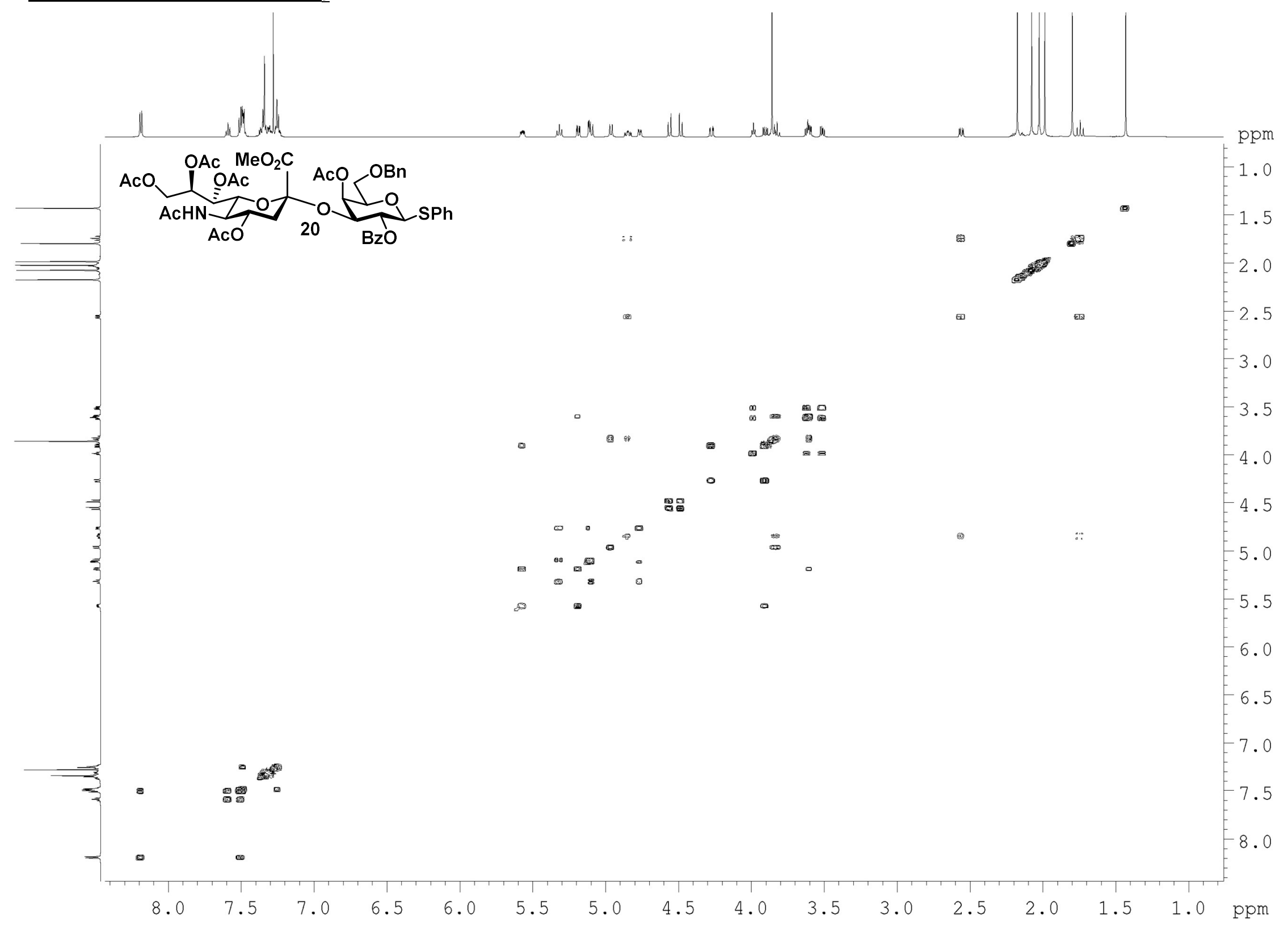




\section{$\underline{\mathrm{HSQC} N M R ; 600 \mathrm{MHz} ; \mathrm{CDCl}_{3}}$}

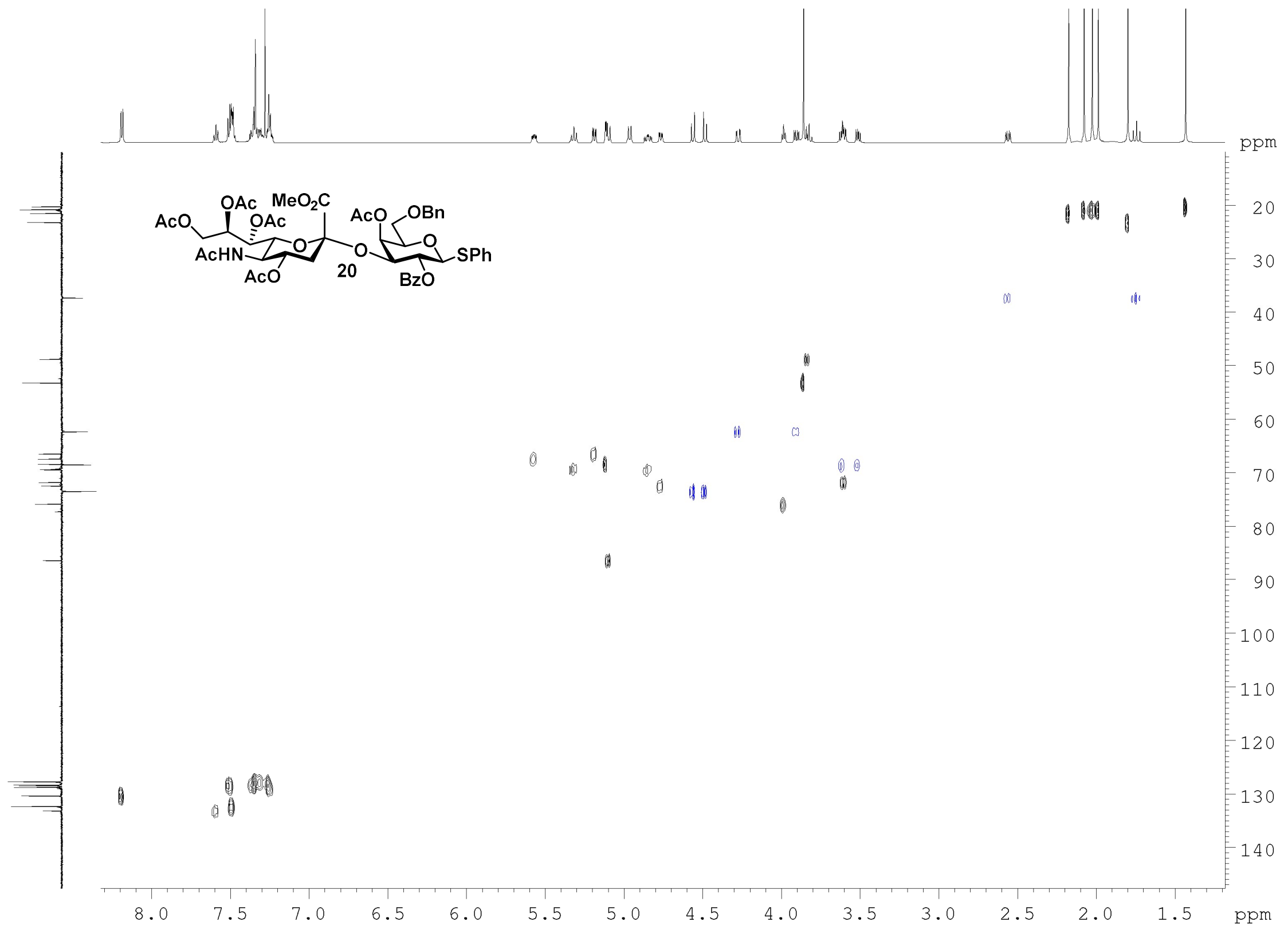




\section{$\underline{\mathrm{HMBC} N M R ; 600 \mathrm{MHz} ; \mathrm{CDCl}_{3}}$}

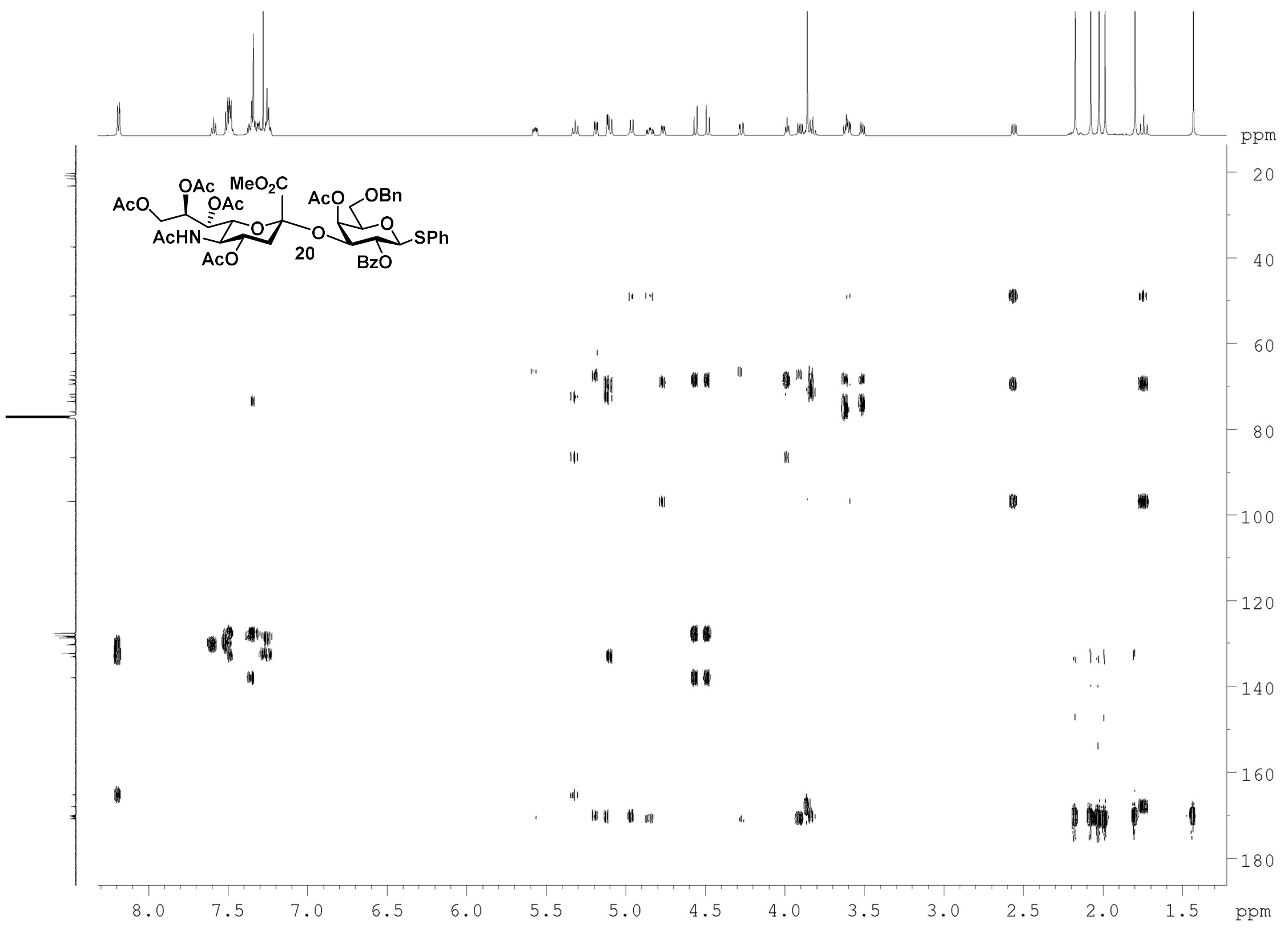




\section{$\underline{{ }^{1} \mathrm{H} N M R ; 600 \mathrm{MHz} ; \mathrm{CDCl}_{3}}$}

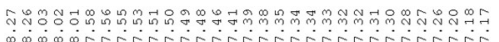

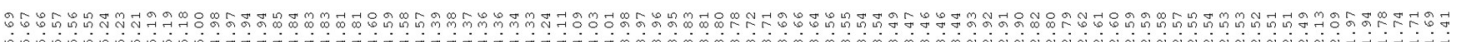

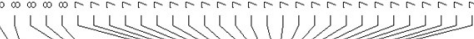
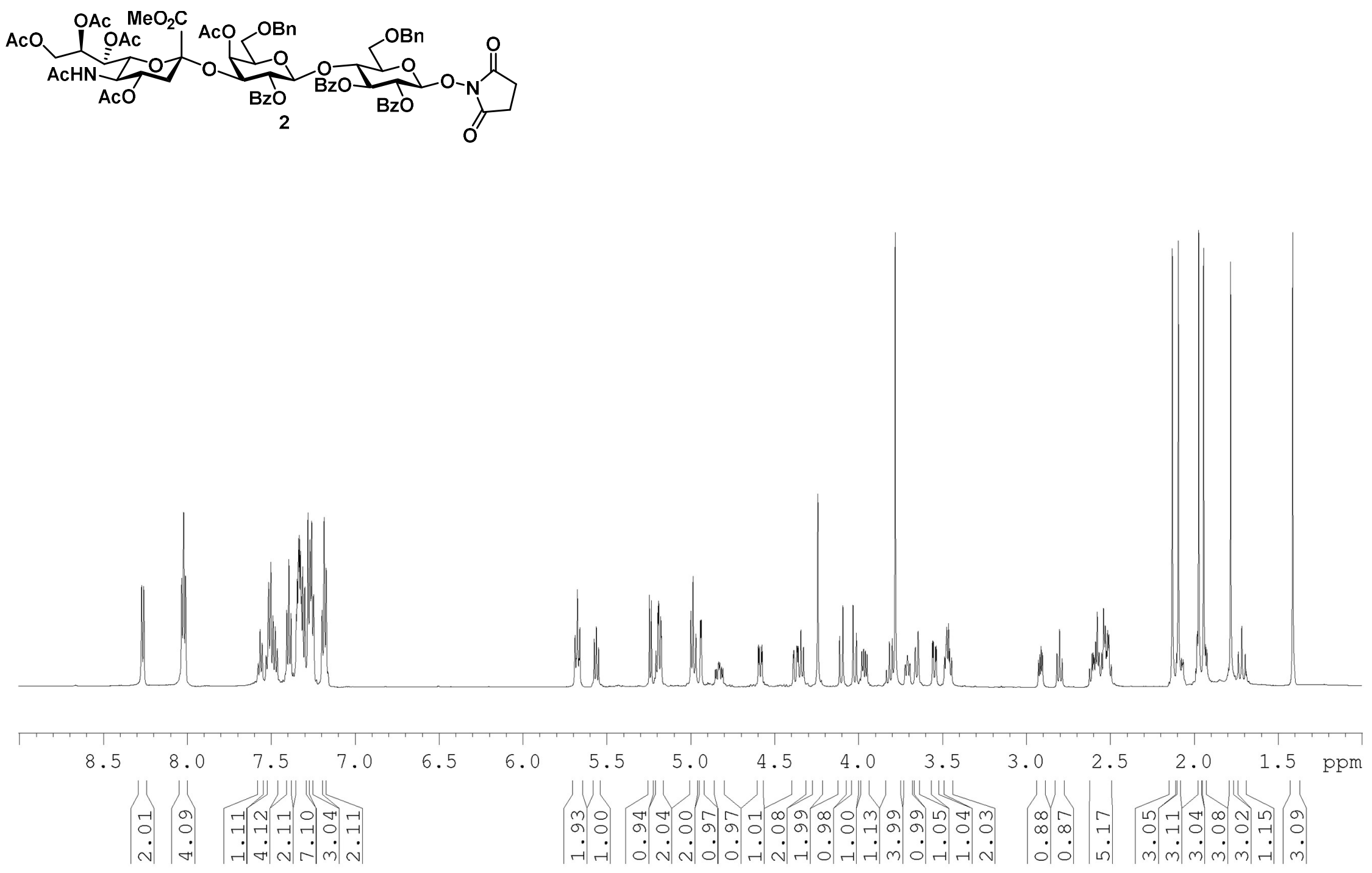


\section{${ }^{13} \mathrm{C} \mathrm{NMR} ; 150 \mathrm{MHz} ; \mathrm{CDCl}_{3}$}
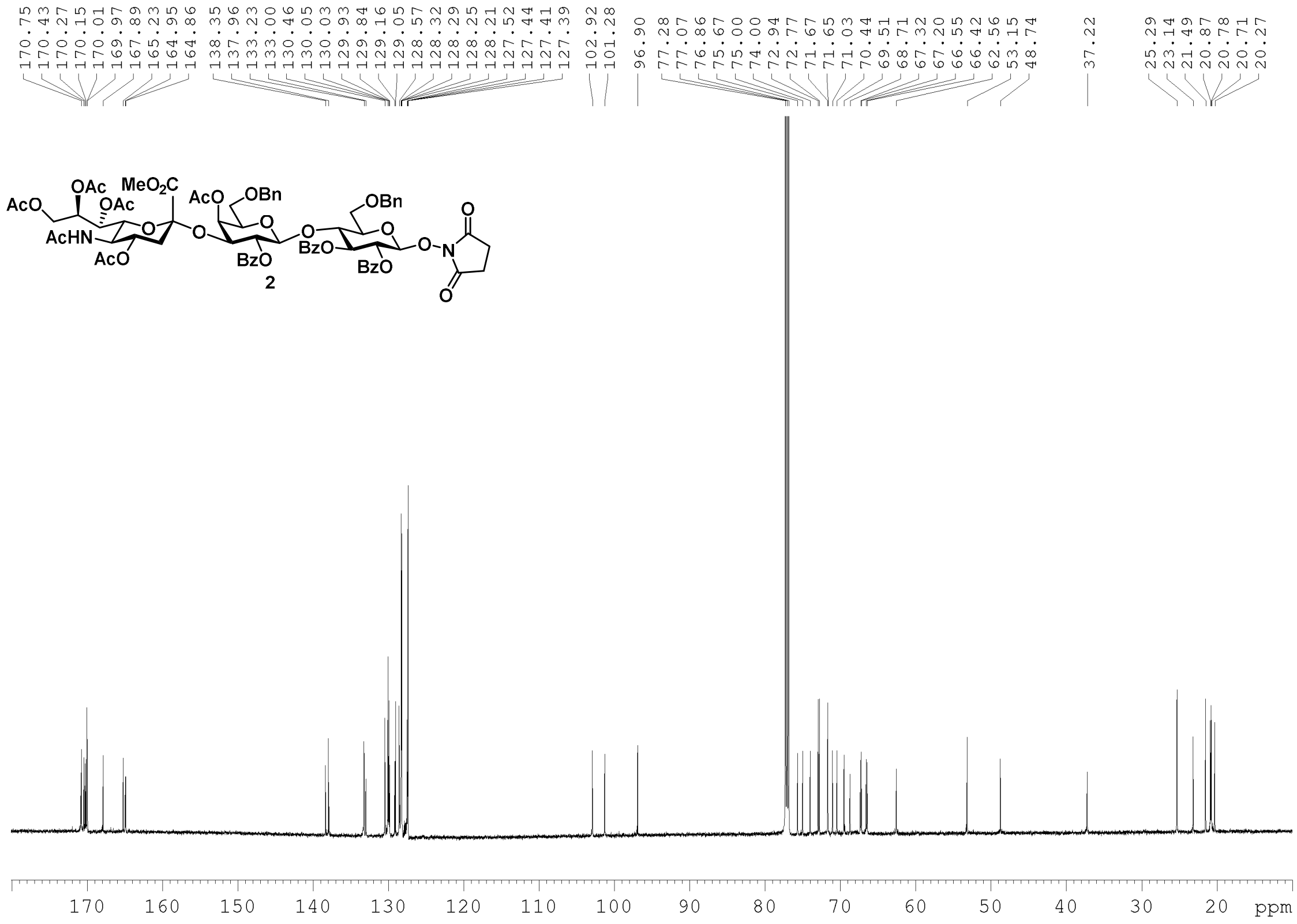


\section{DEPT NMR; $150 \mathrm{MHz} ; \mathrm{CDCl}_{3}$}

m가의

mं

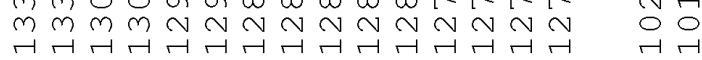

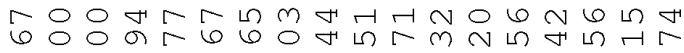

मी

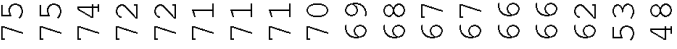

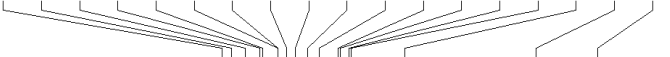

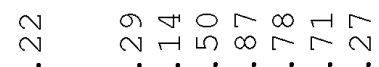

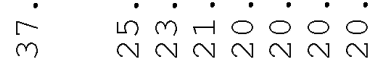
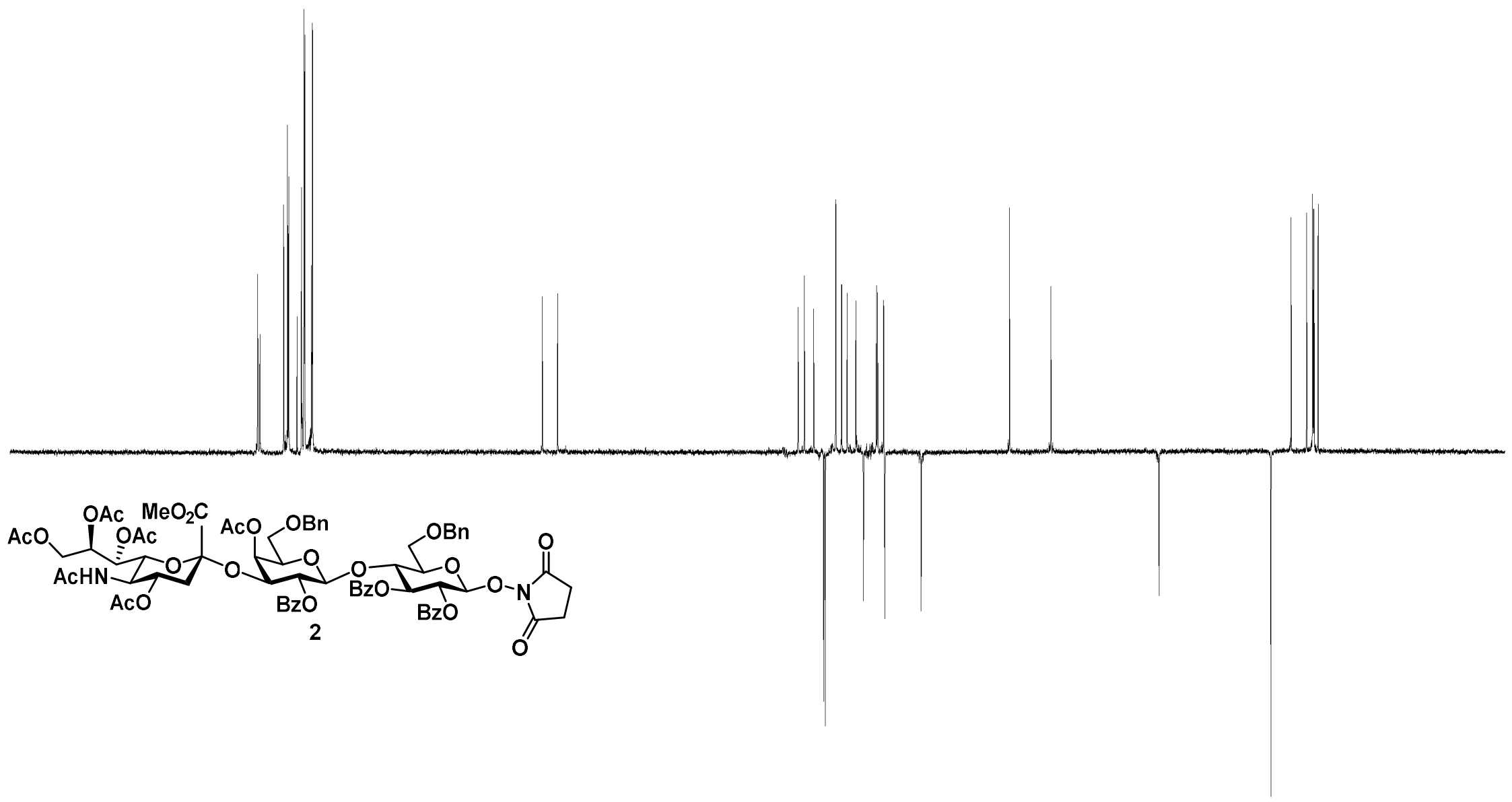

\section{0}

60

50

40

30

$2010 \quad$ ppm 


\section{$\underline{\text { COSY NMR; } 600 \mathrm{MHz} ; \mathrm{CDCl}_{3}}$}

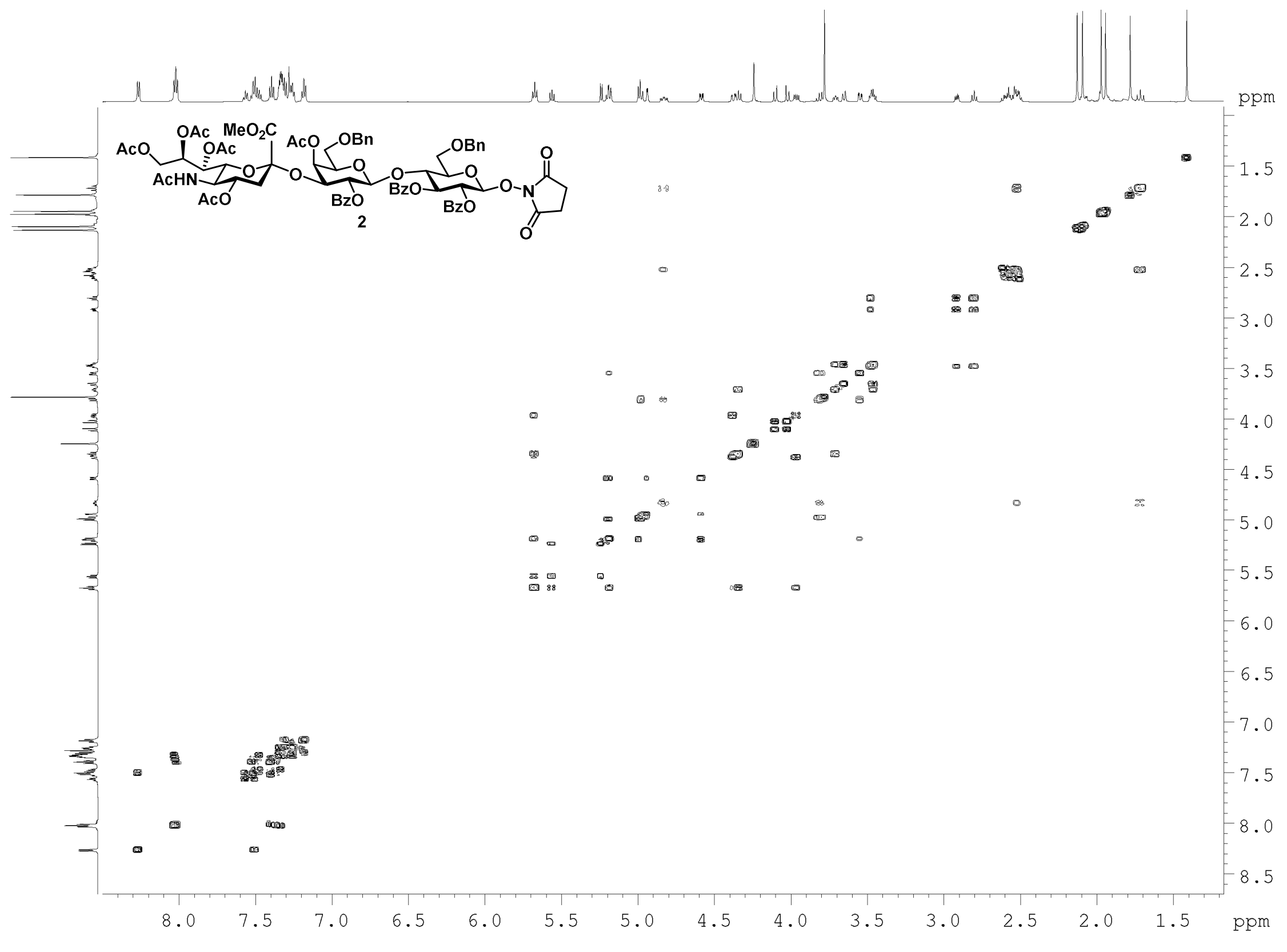




\section{$\underline{\mathrm{HSQC} N M R ; 600 \mathrm{MHz} ; \mathrm{CDCl}_{3}}$}

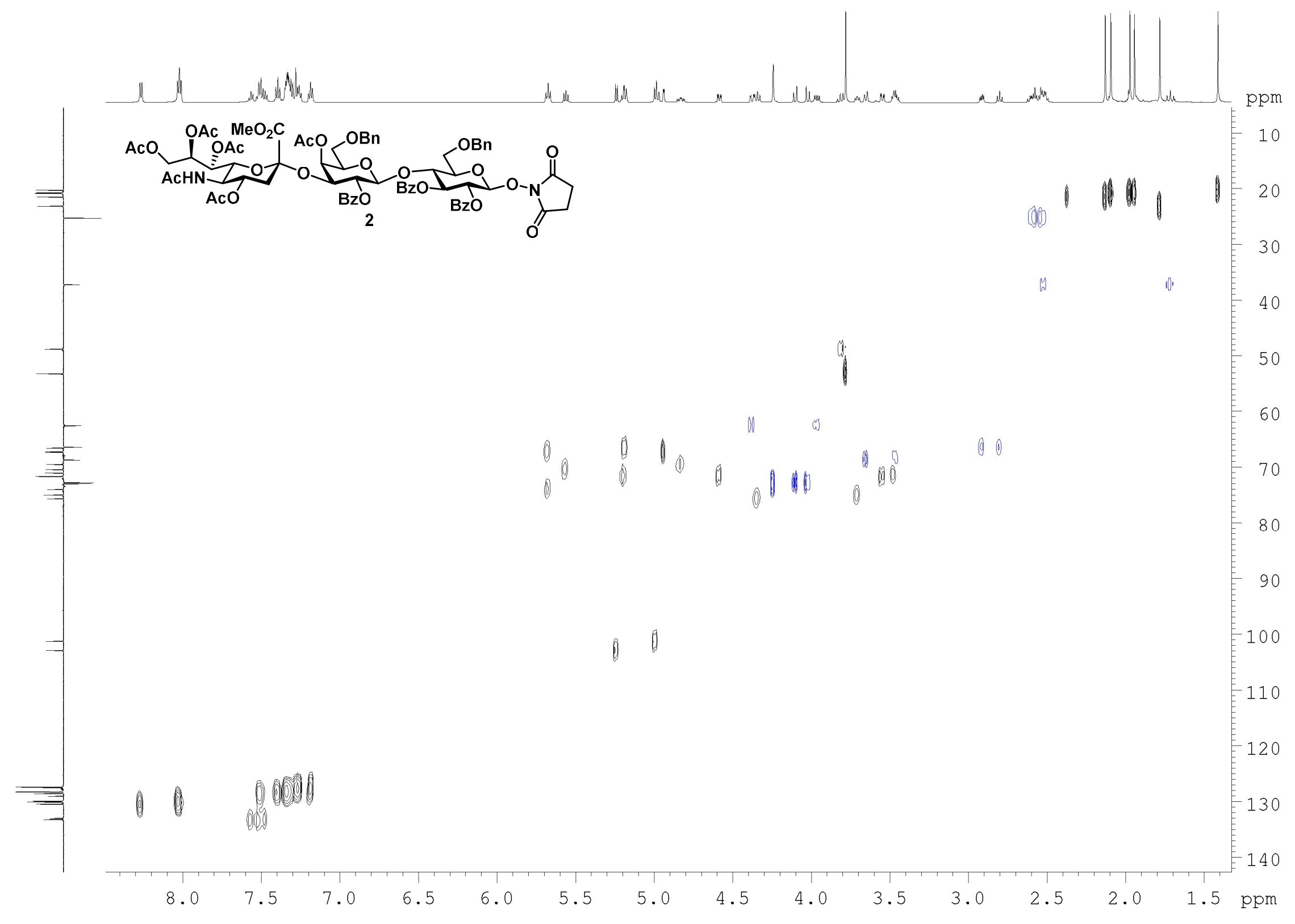




\section{$\underline{\mathrm{HMBC} N M R ; 600 \mathrm{MHz} ; \mathrm{CDCl}_{3}}$}

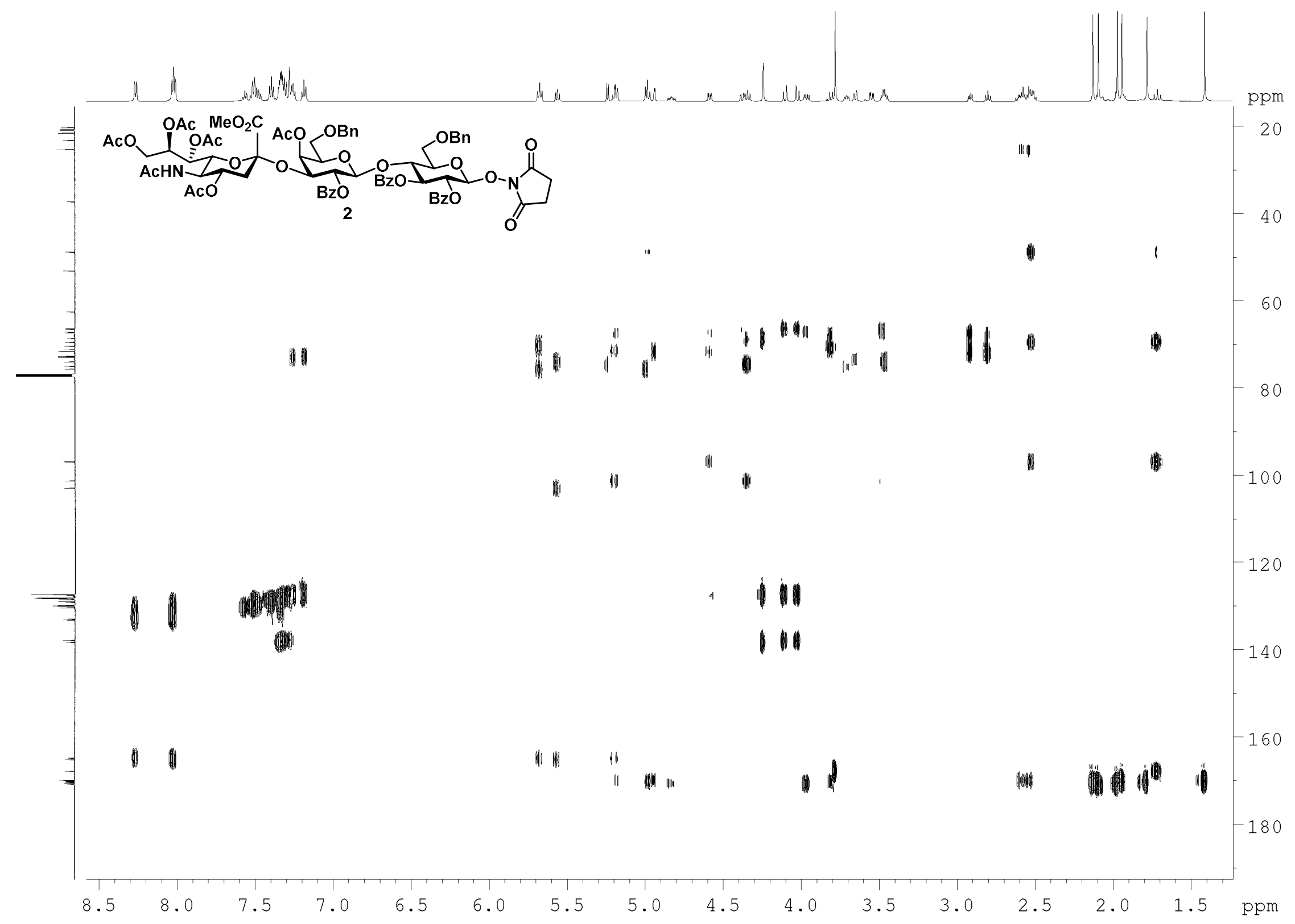




\section{${ }^{1} \mathrm{H} \mathrm{NMR} ; 600 \mathrm{MHz} ; \mathrm{CDCl}_{3}$}

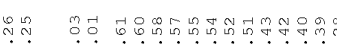

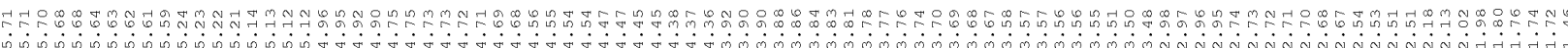
का
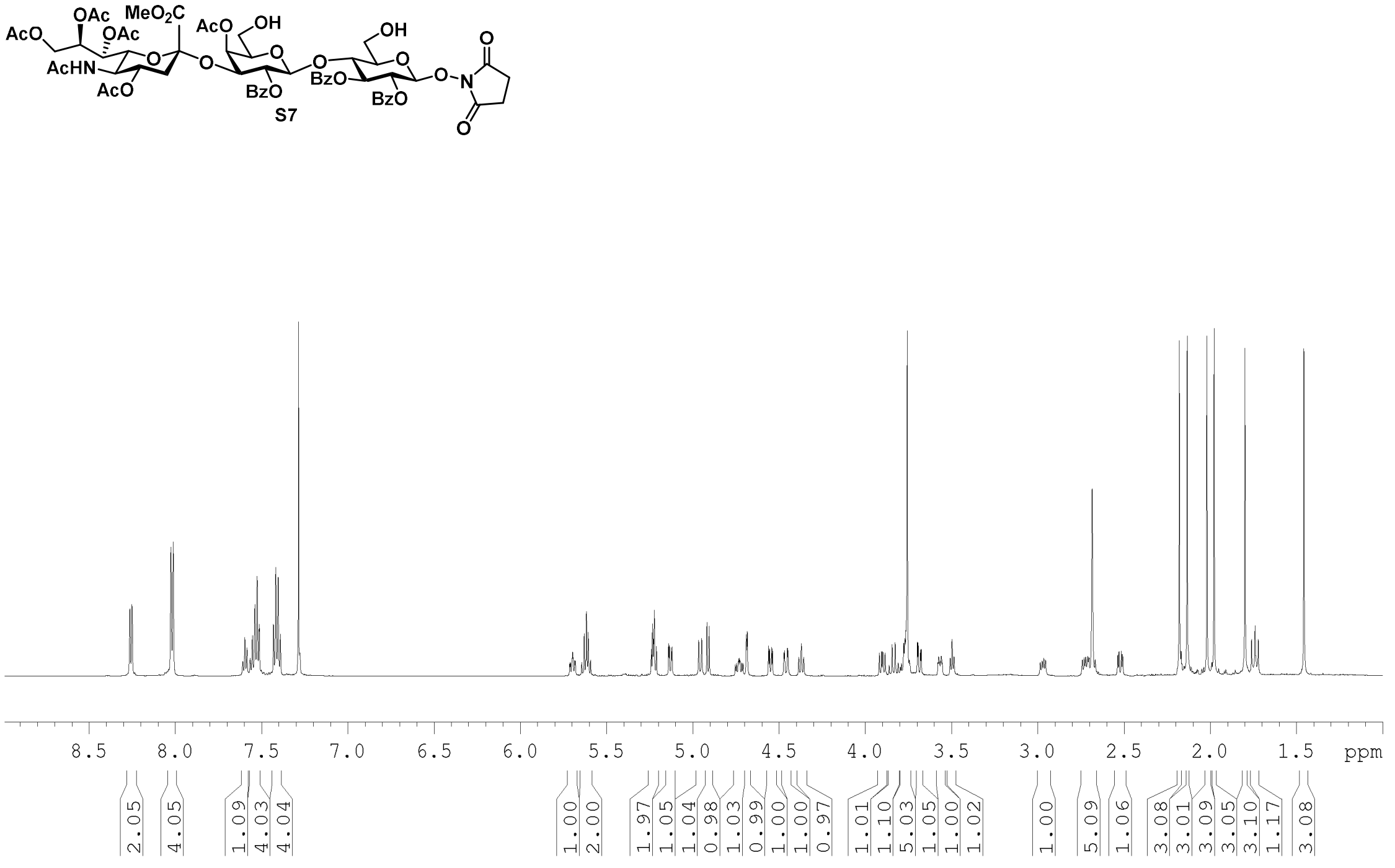


\section{${ }^{13} \mathrm{C} \mathrm{NMR} ; 150 \mathrm{MHz} ; \mathrm{CDCl}_{3}$}

m

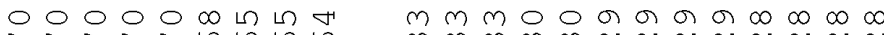

ristrager

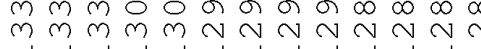

L्याओ

เூ

H०

o o r n

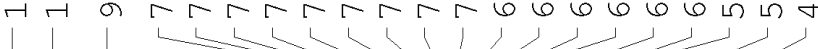

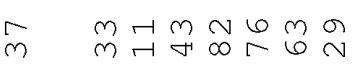

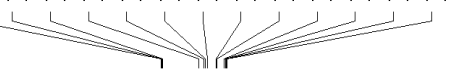

|

ம

$\mathrm{nn} N \mathrm{~N} N \mathrm{n}$
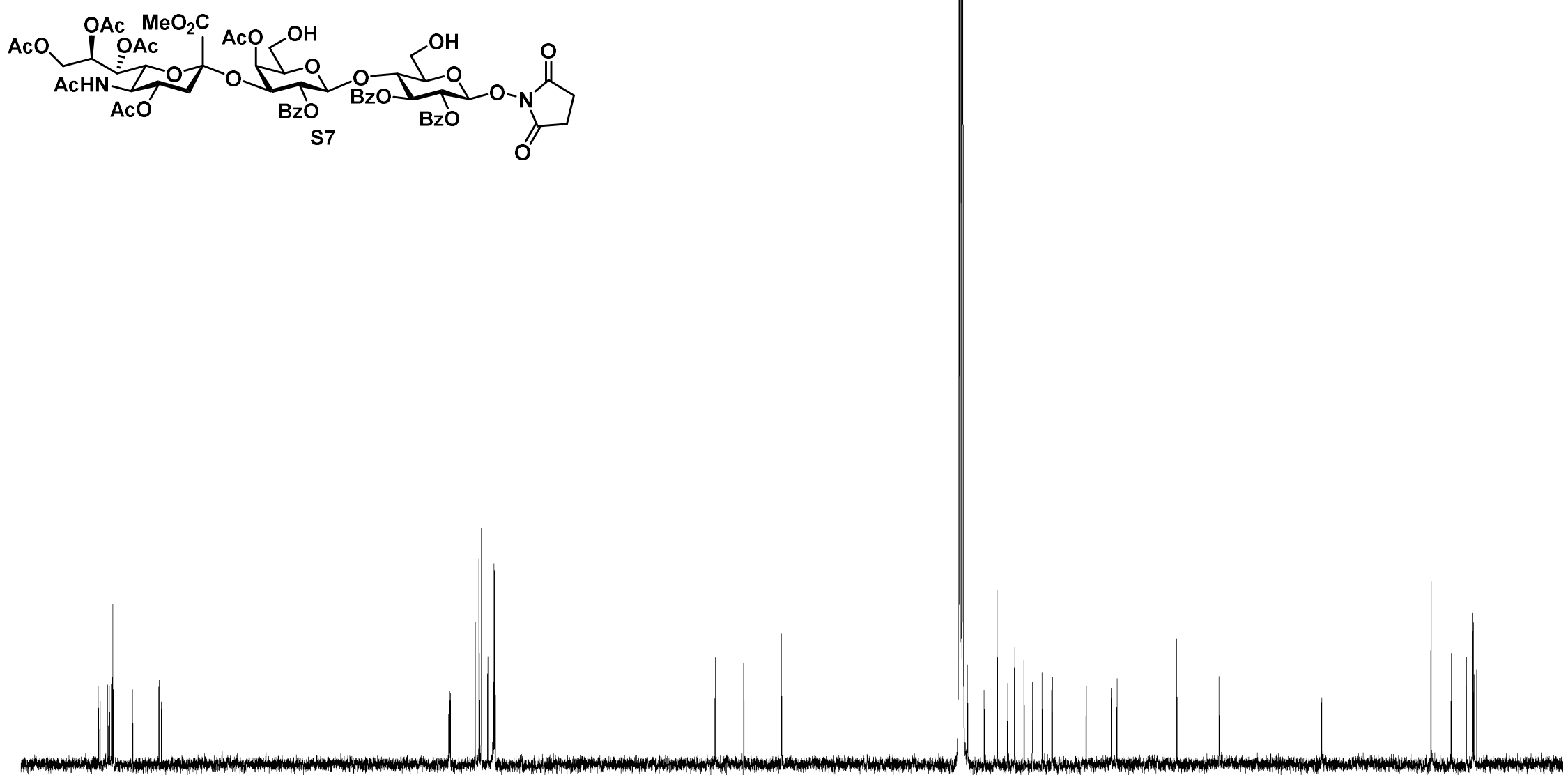

170

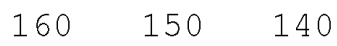

130

120

110

100

90

80

70

60

50

40

30

20 ppm 


\section{$\underline{\text { DEPT NMR; } 150 \mathrm{MHz} ; \mathrm{CDCl}_{3}}$}
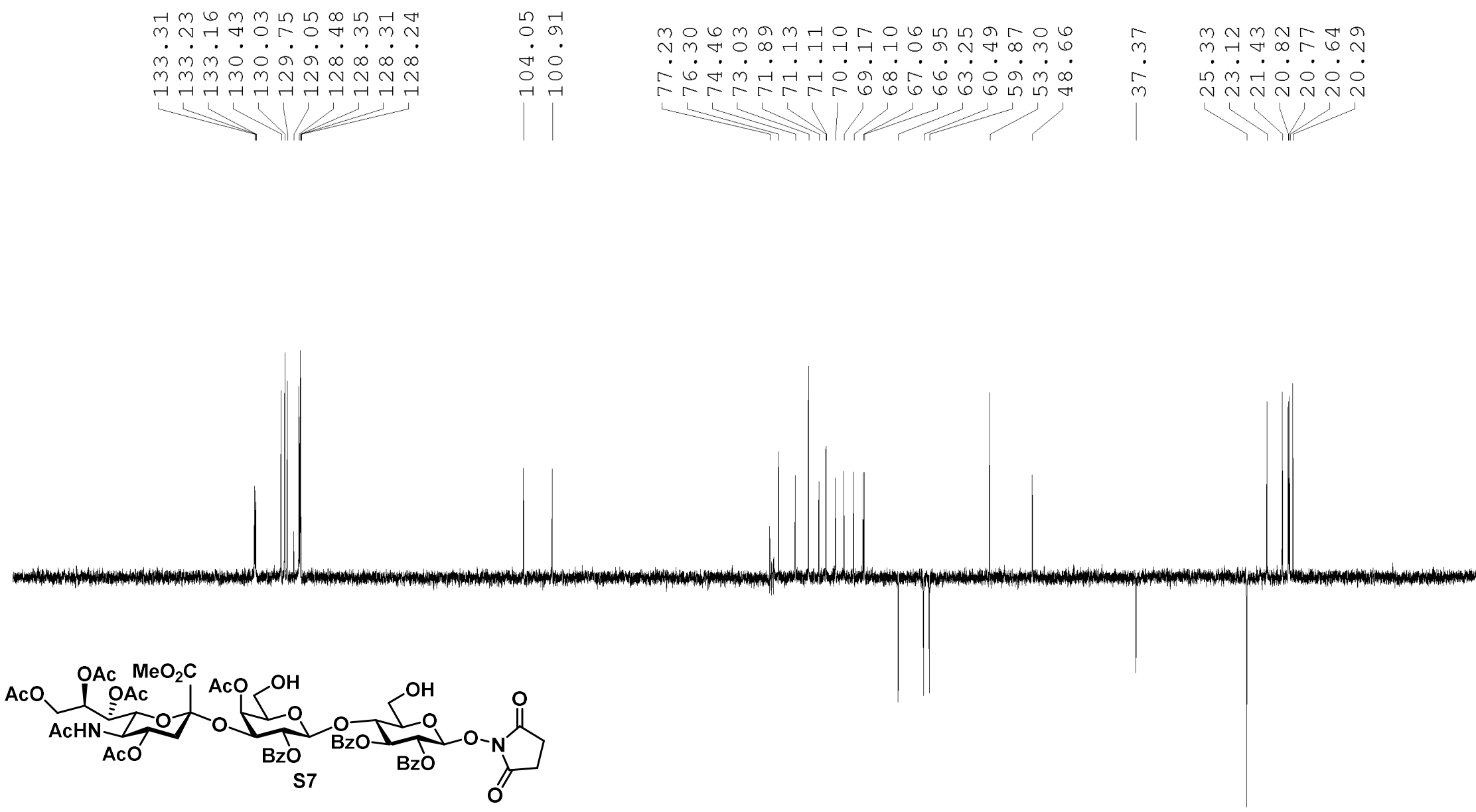
$\underline{\text { COSY NMR; } 600 \mathrm{MHz} ; \mathrm{CDCl}_{3}}$

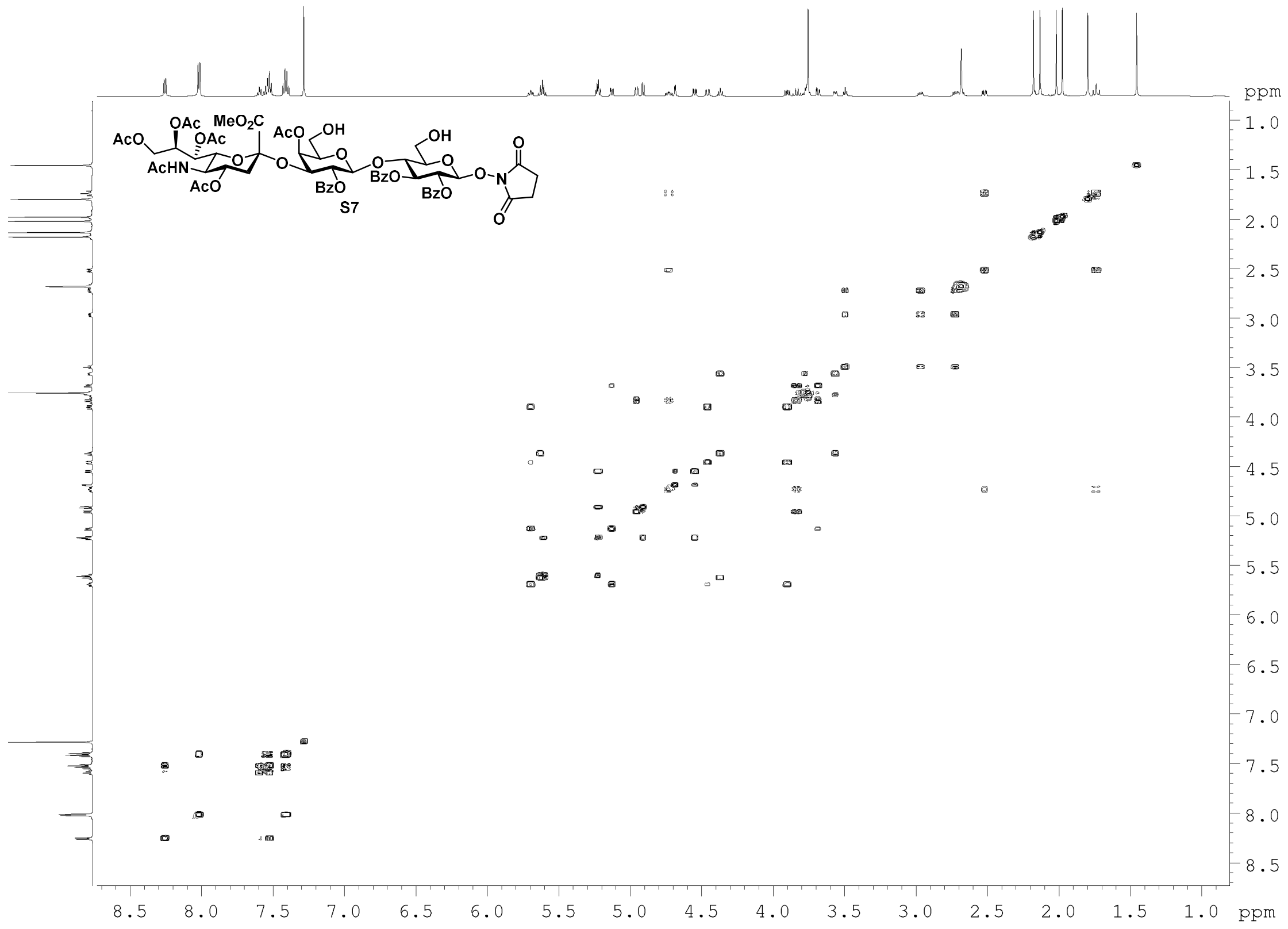




\section{$\underline{\mathrm{HSQC} N M R ; 600 \mathrm{MHz} ; \mathrm{CDCl}_{3}}$}

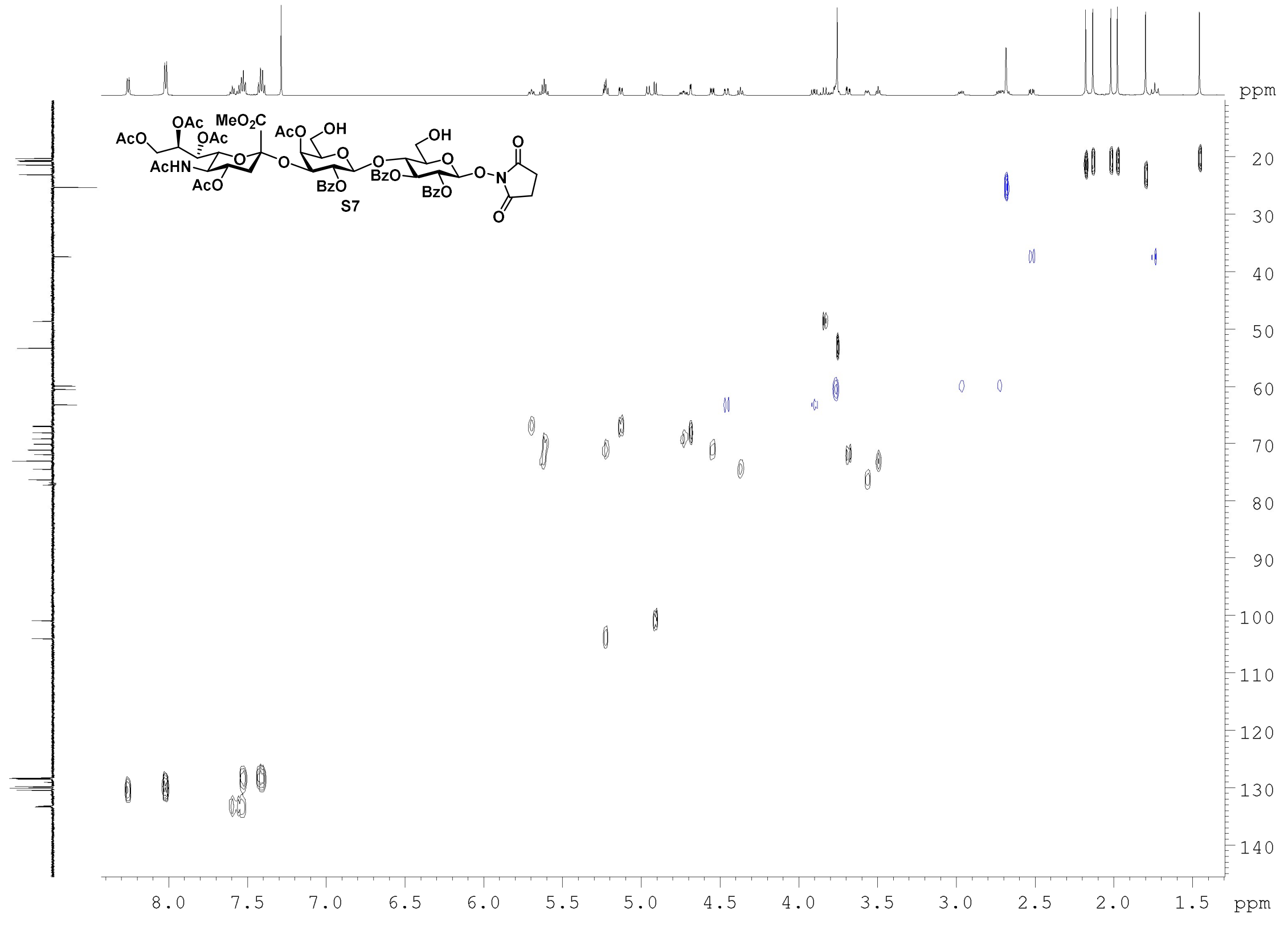




\section{$\underline{\mathrm{HMBC} N M R ; 600 \mathrm{MHz} ; \mathrm{CDCl}_{3}}$}

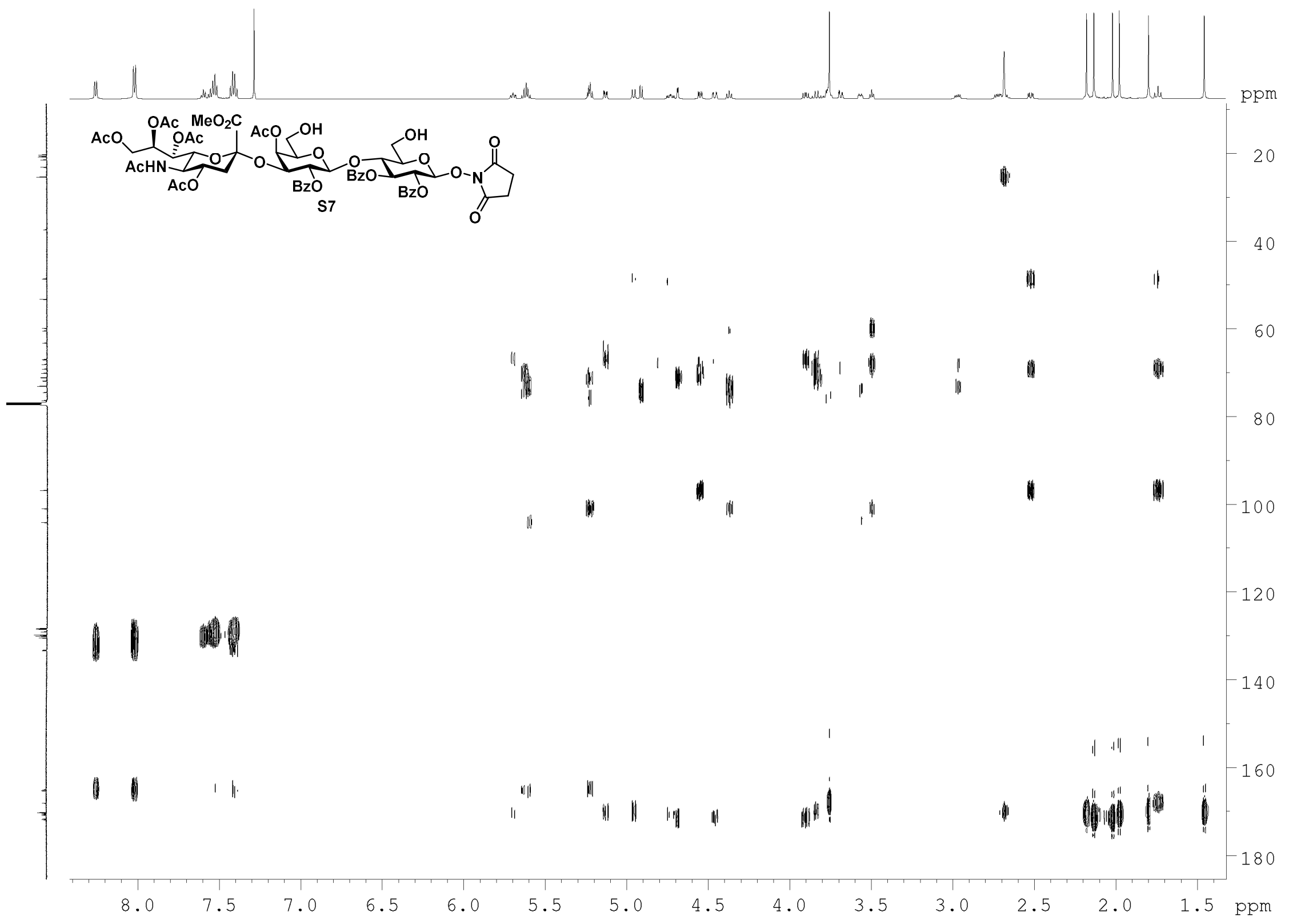




\section{${ }^{1} \mathrm{H}$ NMR; $600 \mathrm{MHz} ; \mathrm{CD}_{3} \mathrm{OD}$}

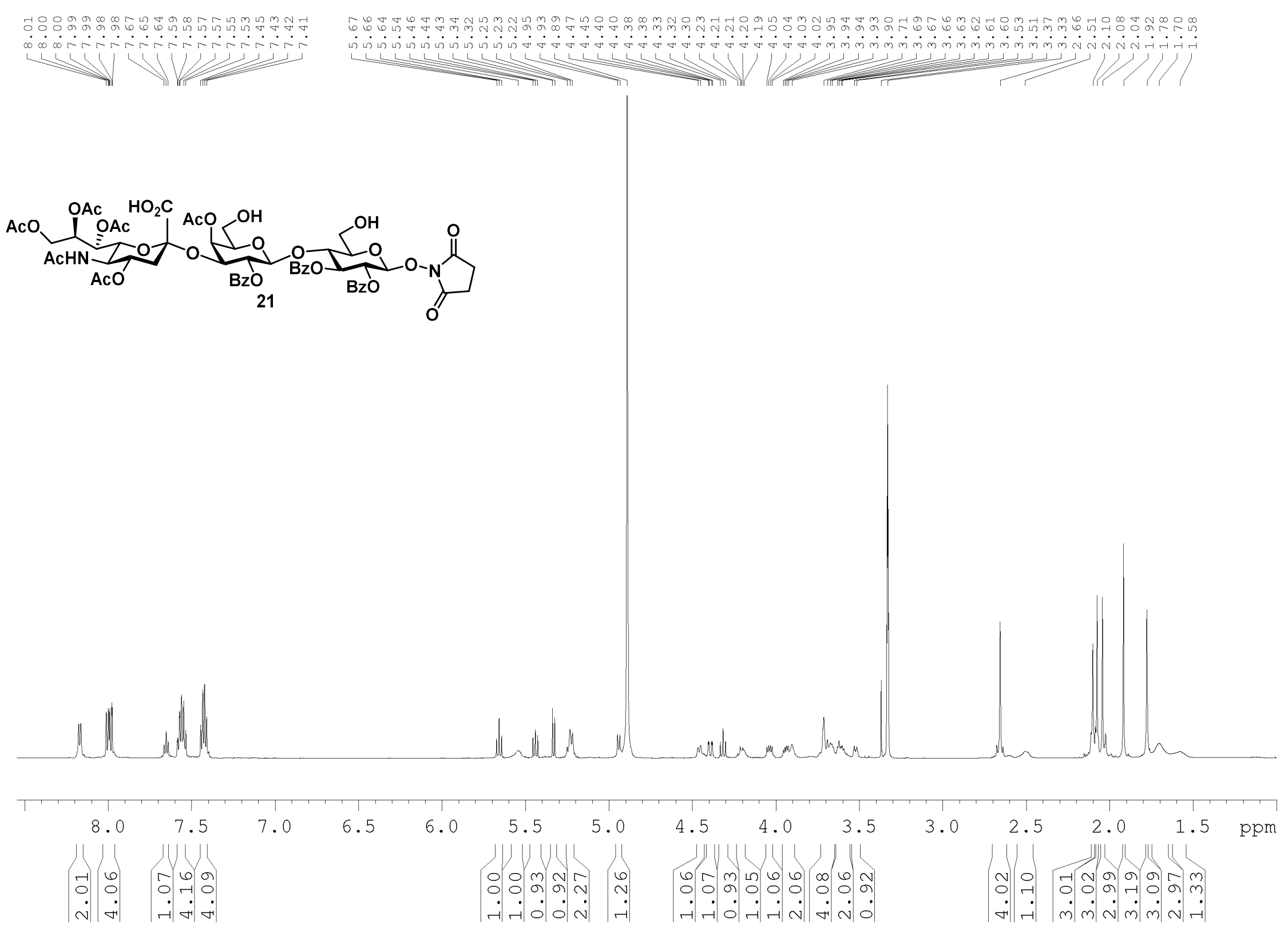




\section{${ }^{13} \mathrm{C}$ NMR; $150 \mathrm{MHz} ; \mathrm{CD}_{3} \mathrm{OD}$}

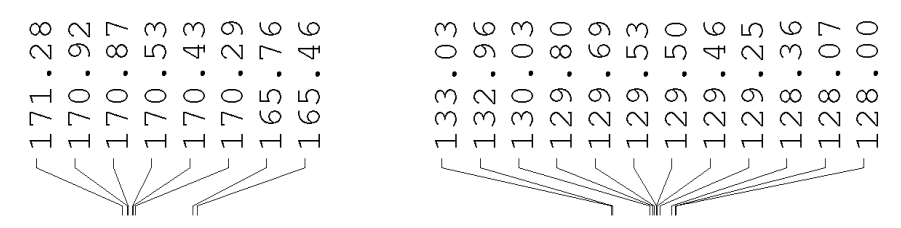

$\stackrel{2}{\circ}$

mं

$\circlearrowleft$

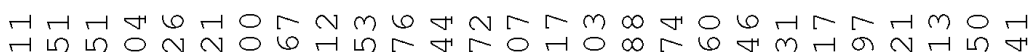

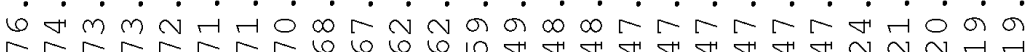
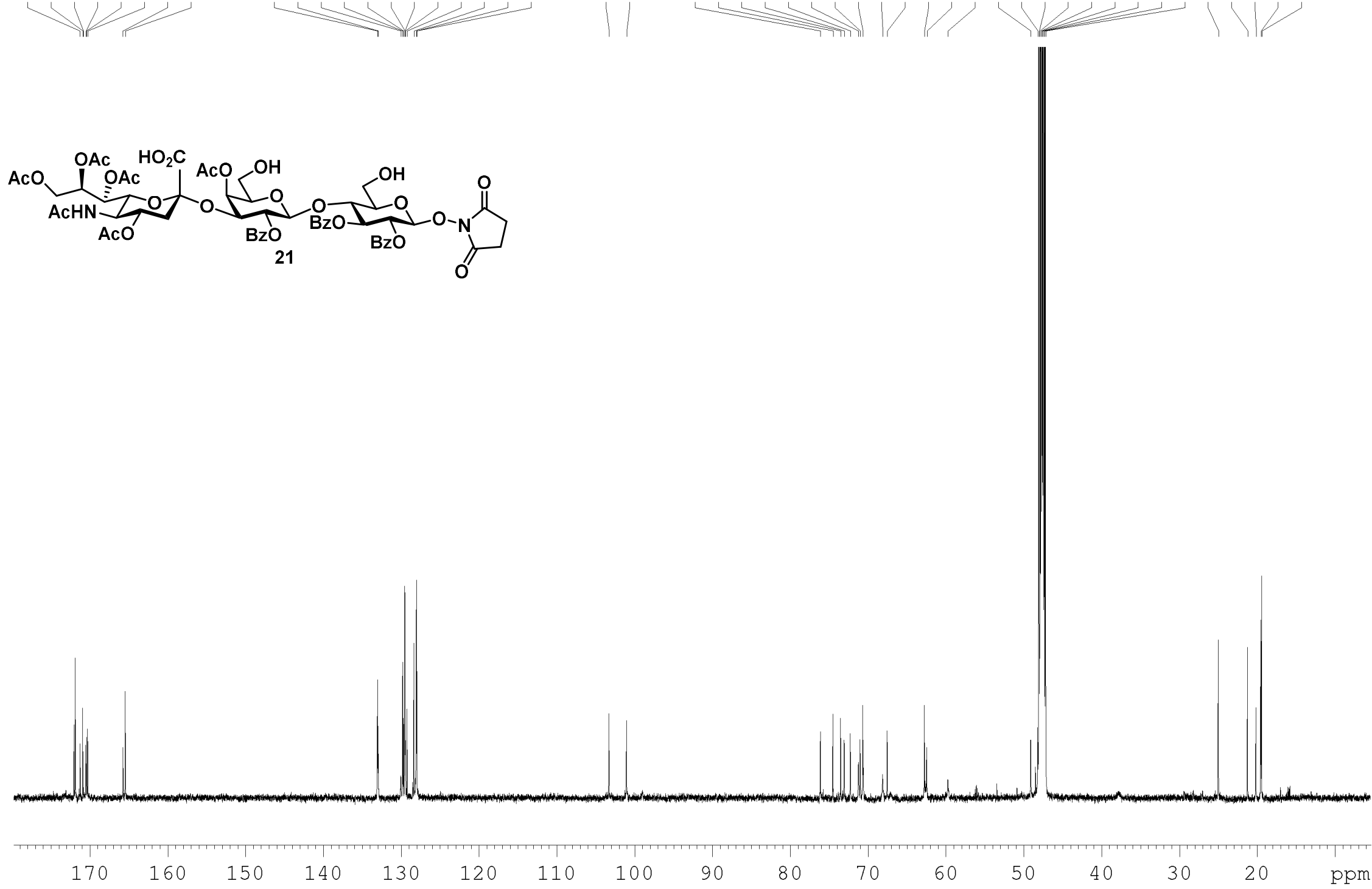


\section{$\underline{\text { DEPT NMR; } 150 \mathrm{MHz} ; \mathrm{CD}_{3} \mathrm{OD}}$}

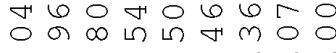

mino

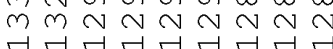

$m 0$

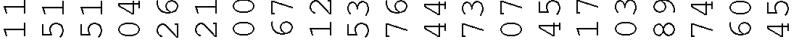

7.

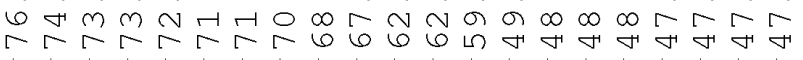

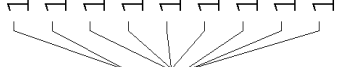

m

L

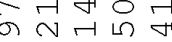
-ं宀कंष
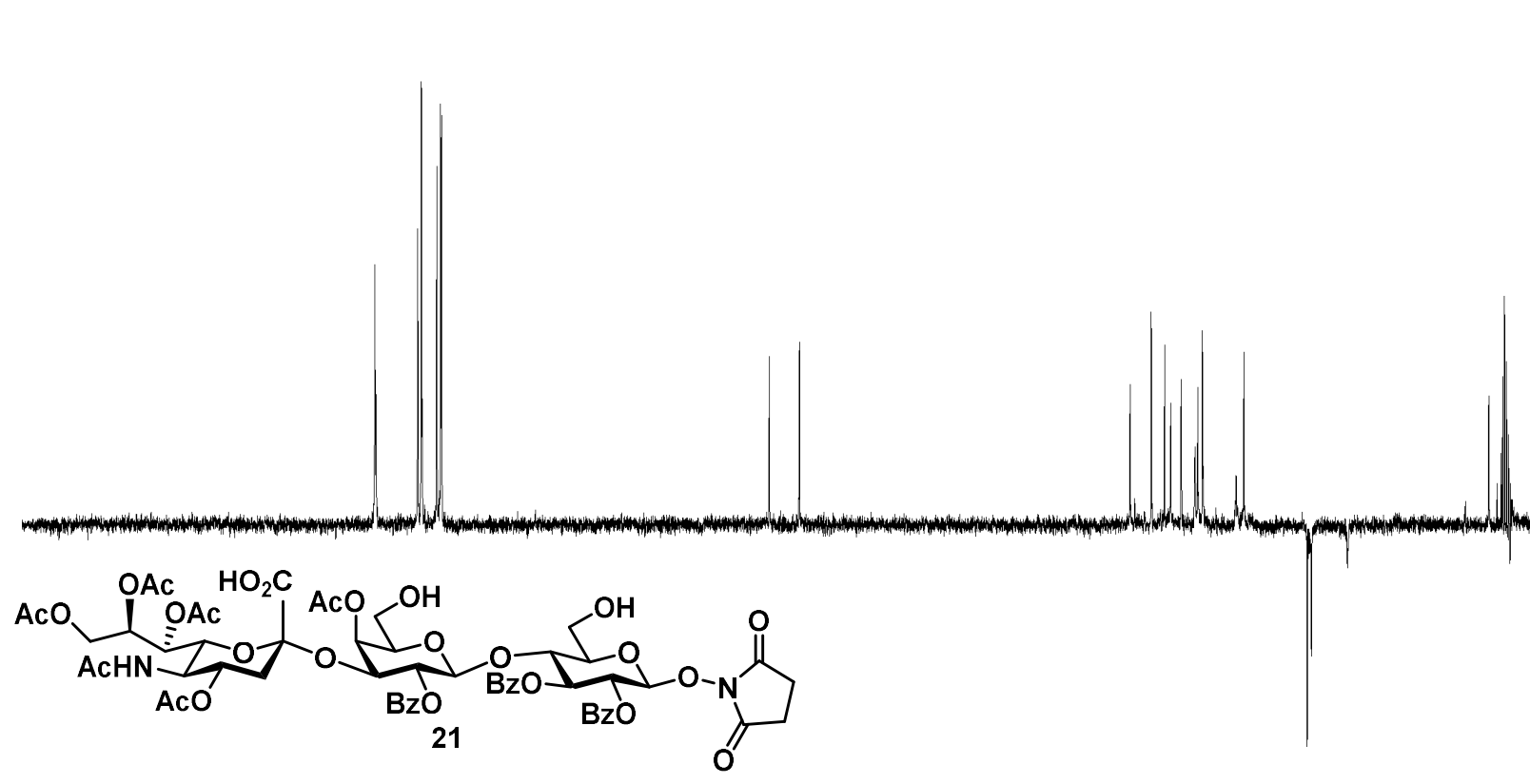


\section{$\underline{\text { COSY NMR; } 600 \mathrm{MHz} ; \mathrm{CD}_{3} \mathrm{OD}}$}

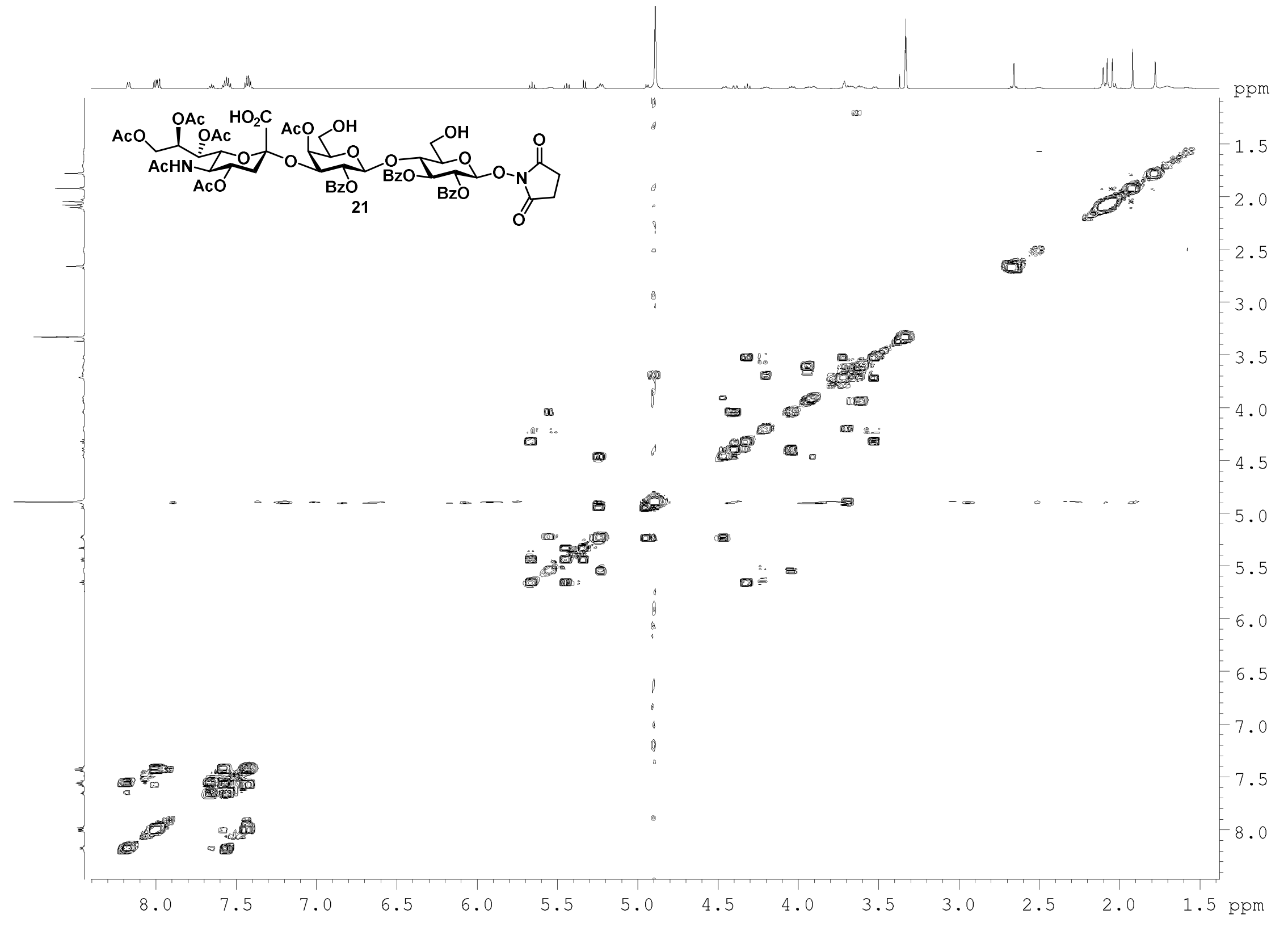




\section{$\underline{\mathrm{HSQC} N M R ; 600 \mathrm{MHz} ; \mathrm{CD}_{3} \mathrm{OD}}$}

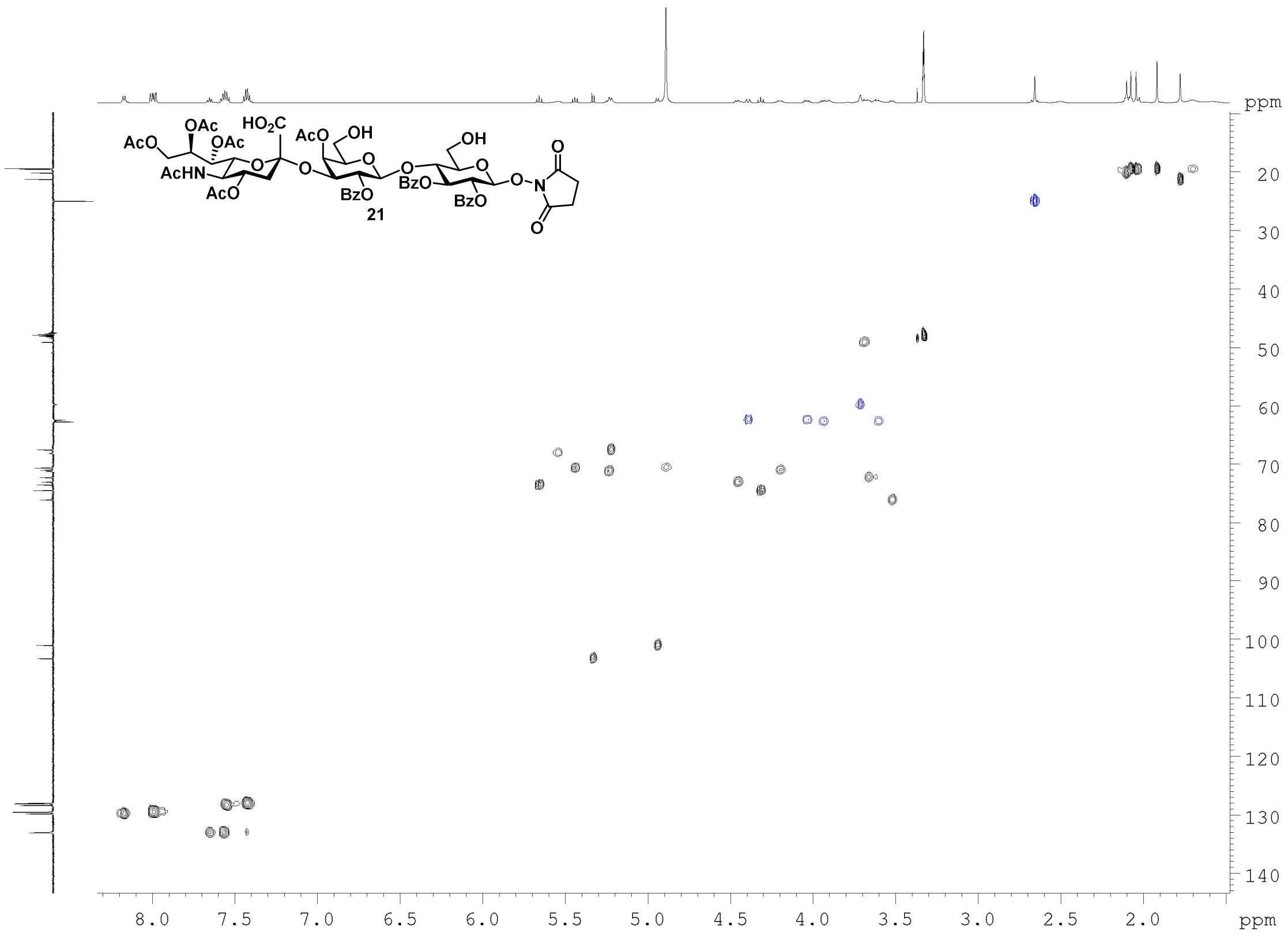


$\underline{\mathrm{HMBC} N M R ; 600 \mathrm{MHz} ; \mathrm{CD}_{3} \mathrm{OD}}$

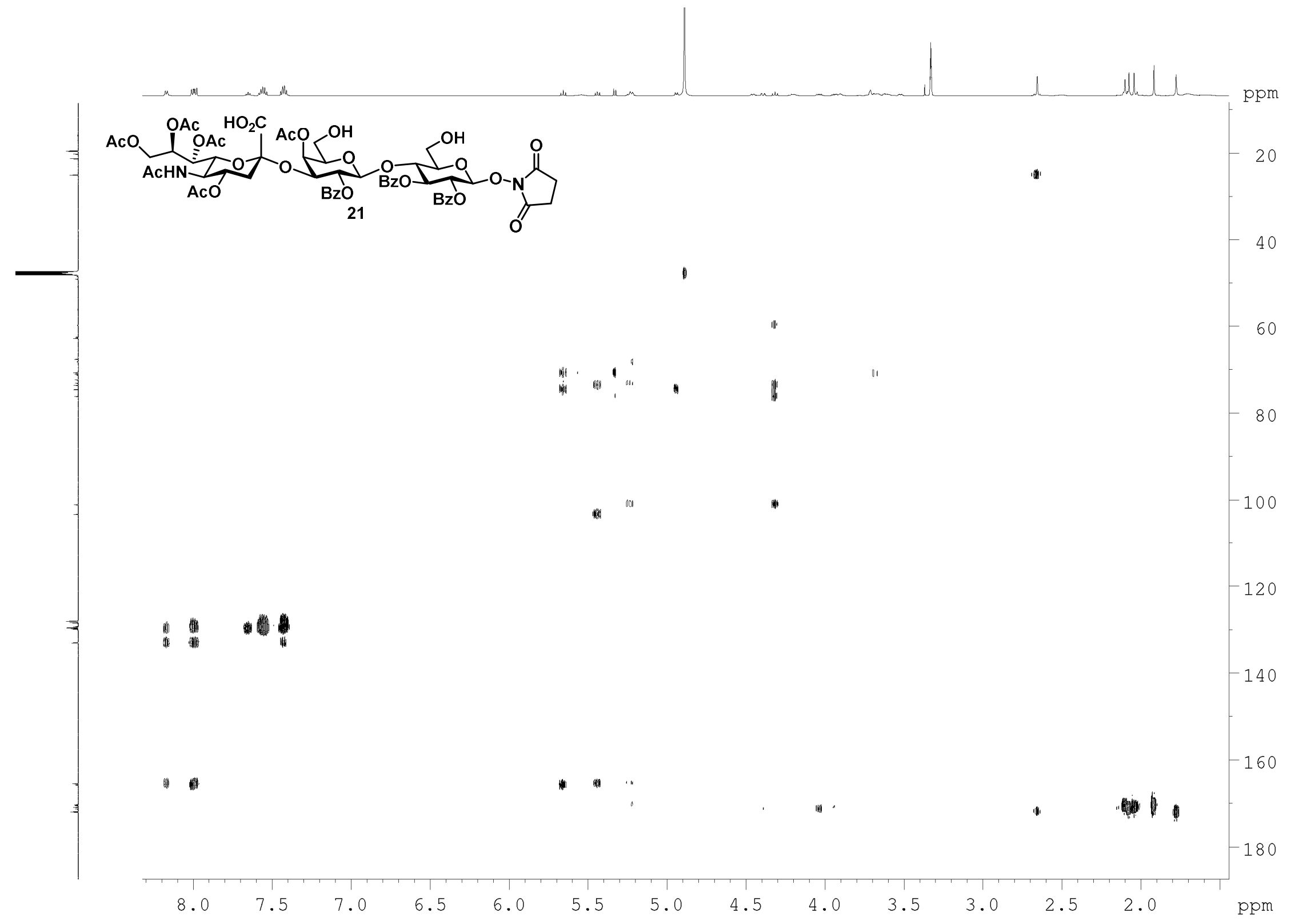




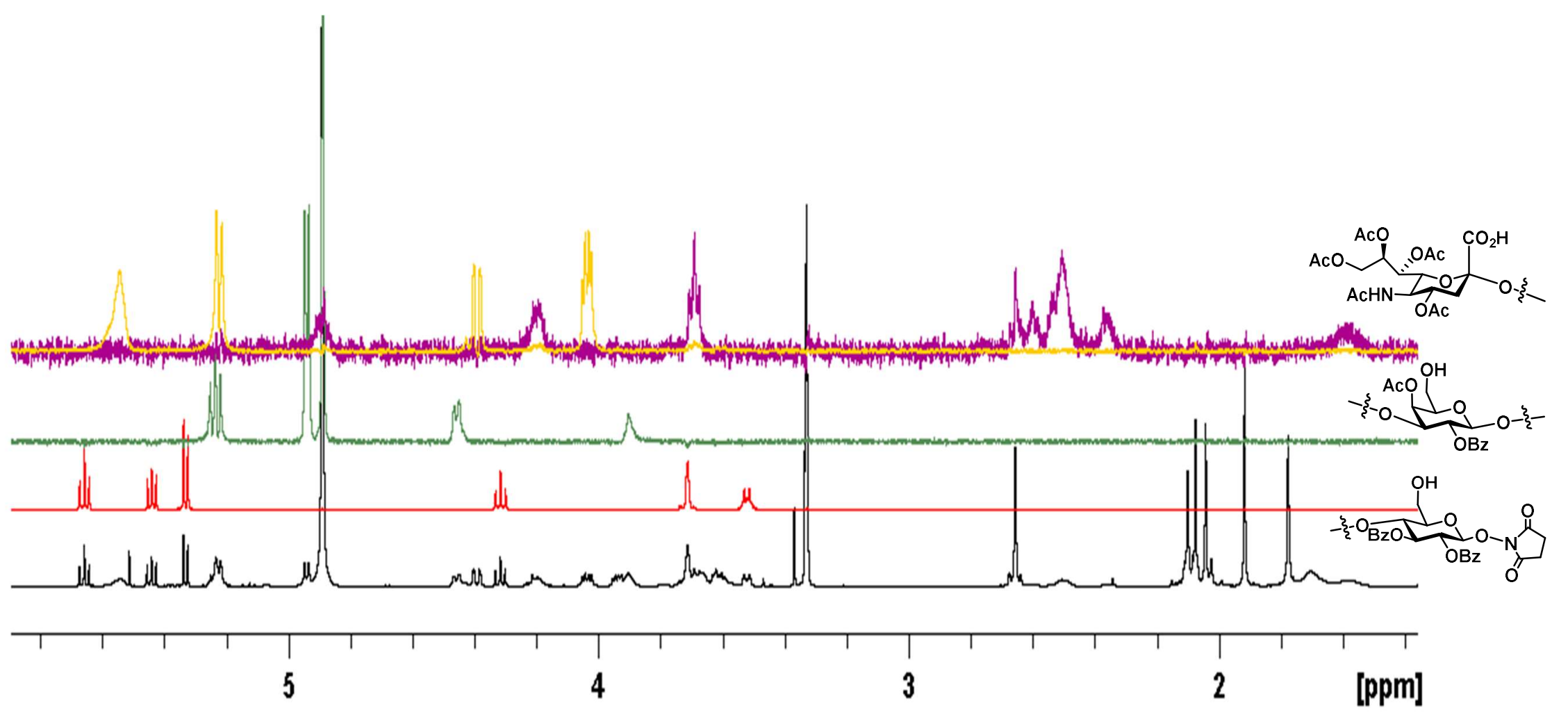


${ }^{1} \mathrm{H}$ NMR; $600 \mathrm{MHz} ; \mathrm{D}_{2} \mathrm{O}$

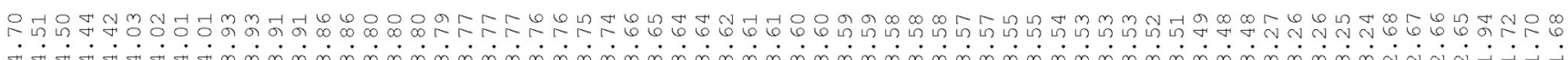
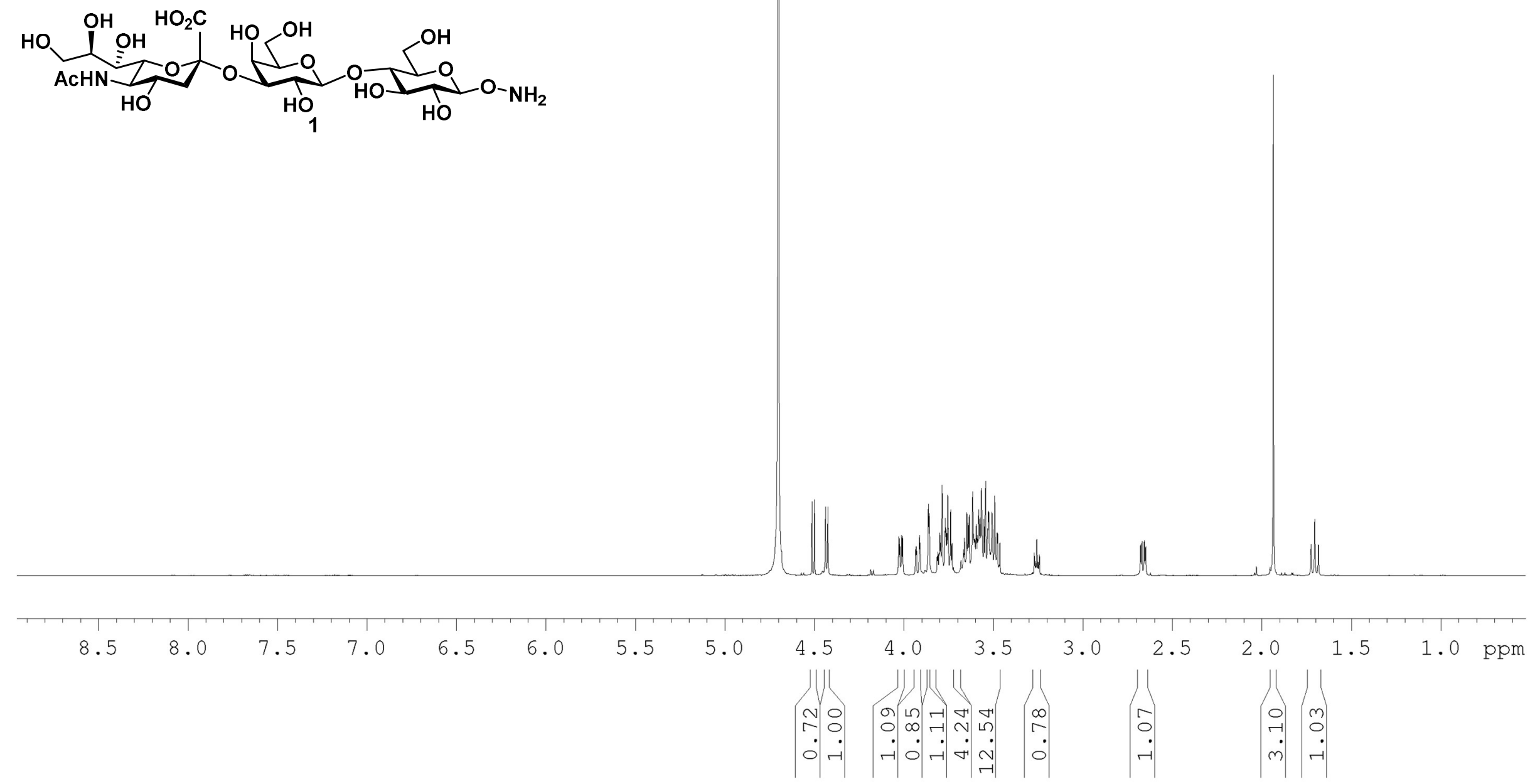


\section{${ }^{1} \mathrm{H}$ NMR; $600 \mathrm{MHz} ; \mathrm{D}_{2} \mathrm{O}$}

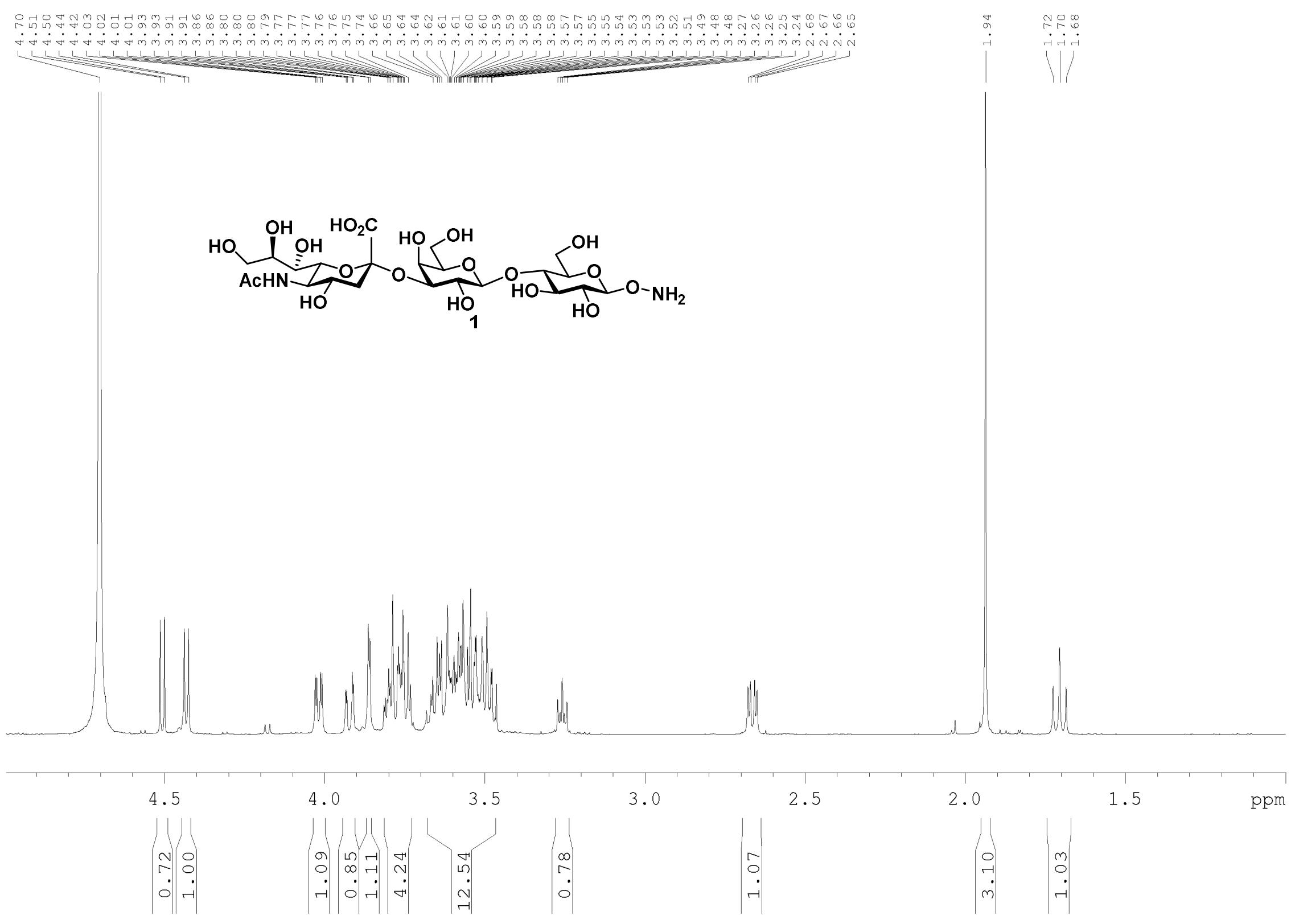


${ }^{13} \mathrm{C}$ NMR; $150 \mathrm{MHz} ; \mathrm{D}_{2} \mathrm{O}$

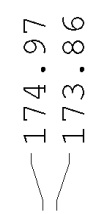

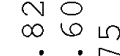

अं $\dot{0}^{r}$

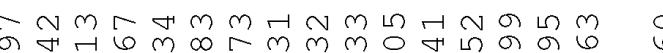

- $\dot{0} \cdot \dot{0} \cdot \dot{0} \cdot \dot{0} \dot{0} \dot{0}$

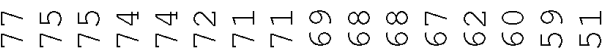

LLL L
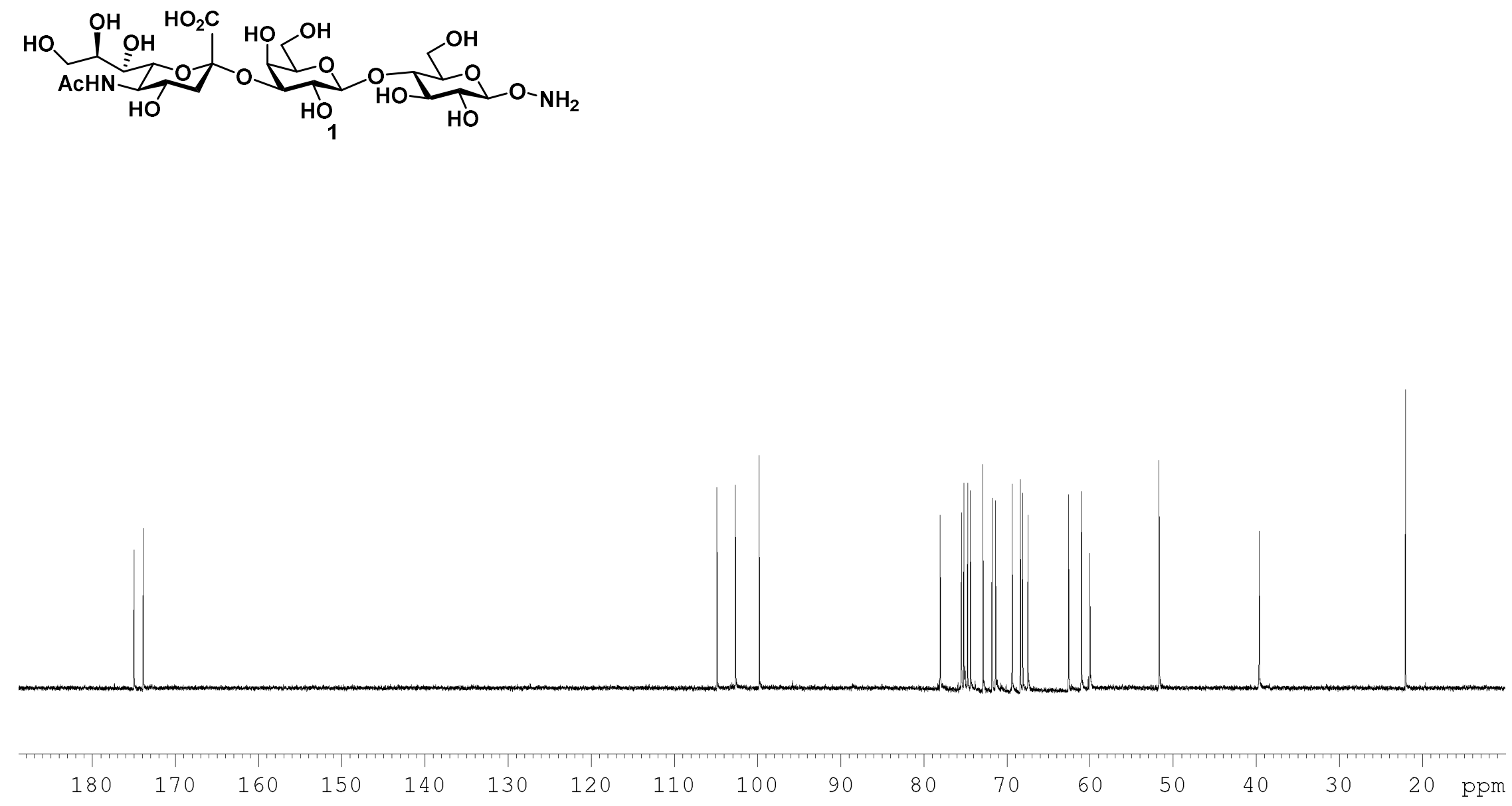
DEPT NMR; $150 \mathrm{MHz} ; \mathrm{D}_{2} \mathrm{O}$

$\begin{array}{ll}m & 0 \\ \infty & 0 \\ \dot{H} & \dot{1} \\ \circ & 0 \\ +1 & -1 \\ \mid & \mid\end{array}$
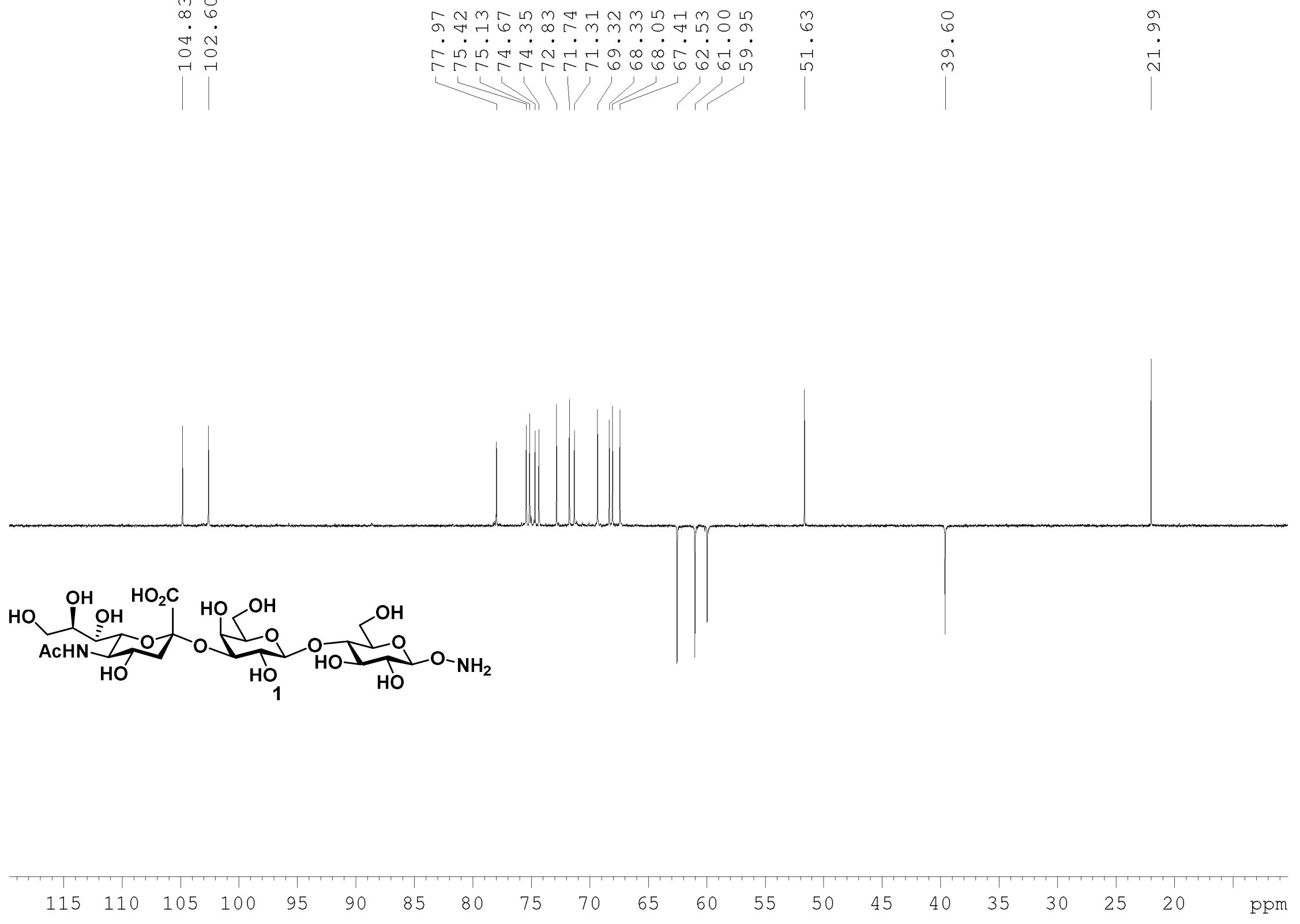

S99 


\section{$\underline{\text { COSY NMR; } 600 \mathrm{MHz} ; \mathrm{D}_{2} \mathrm{O}}$}

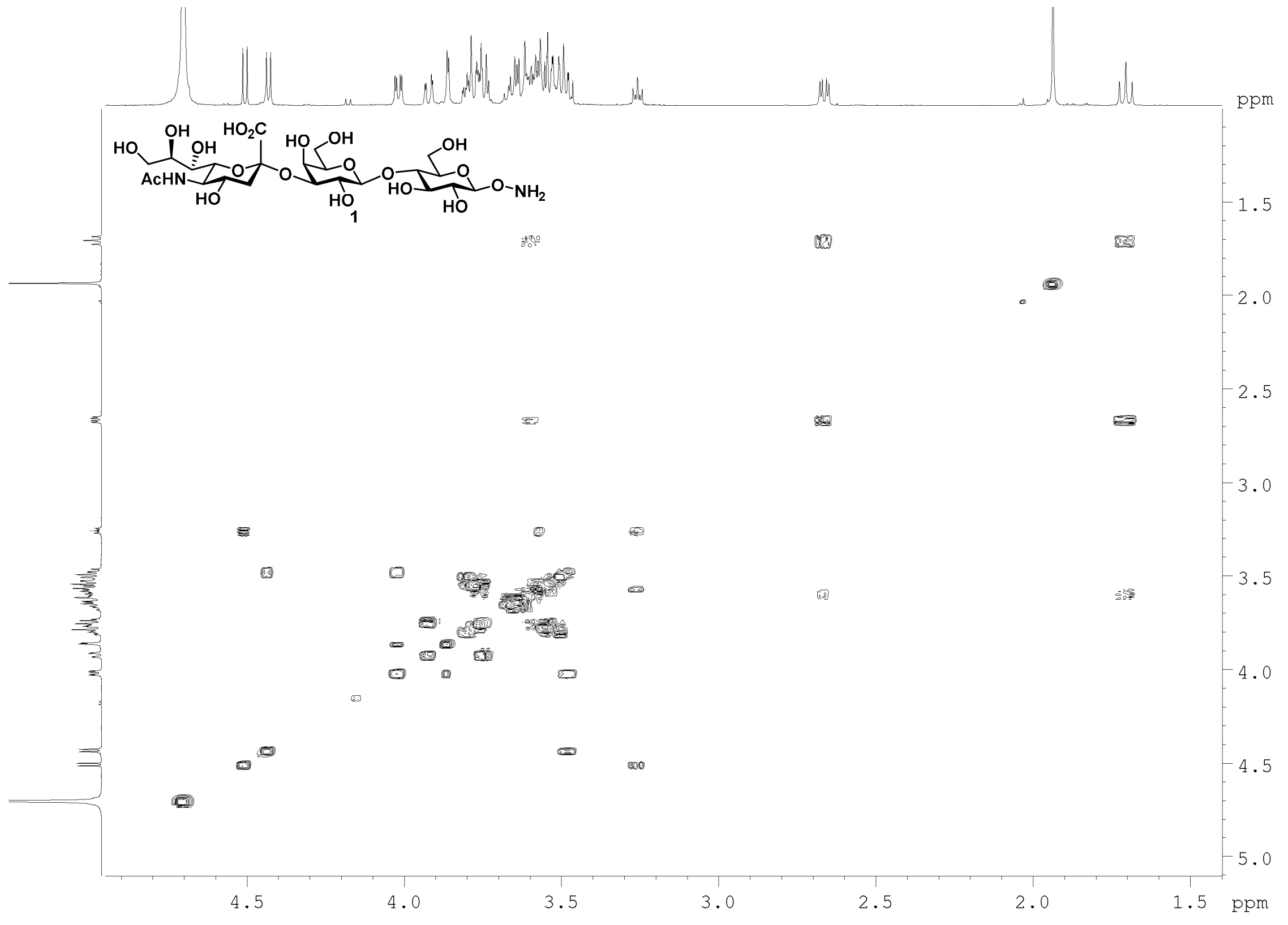




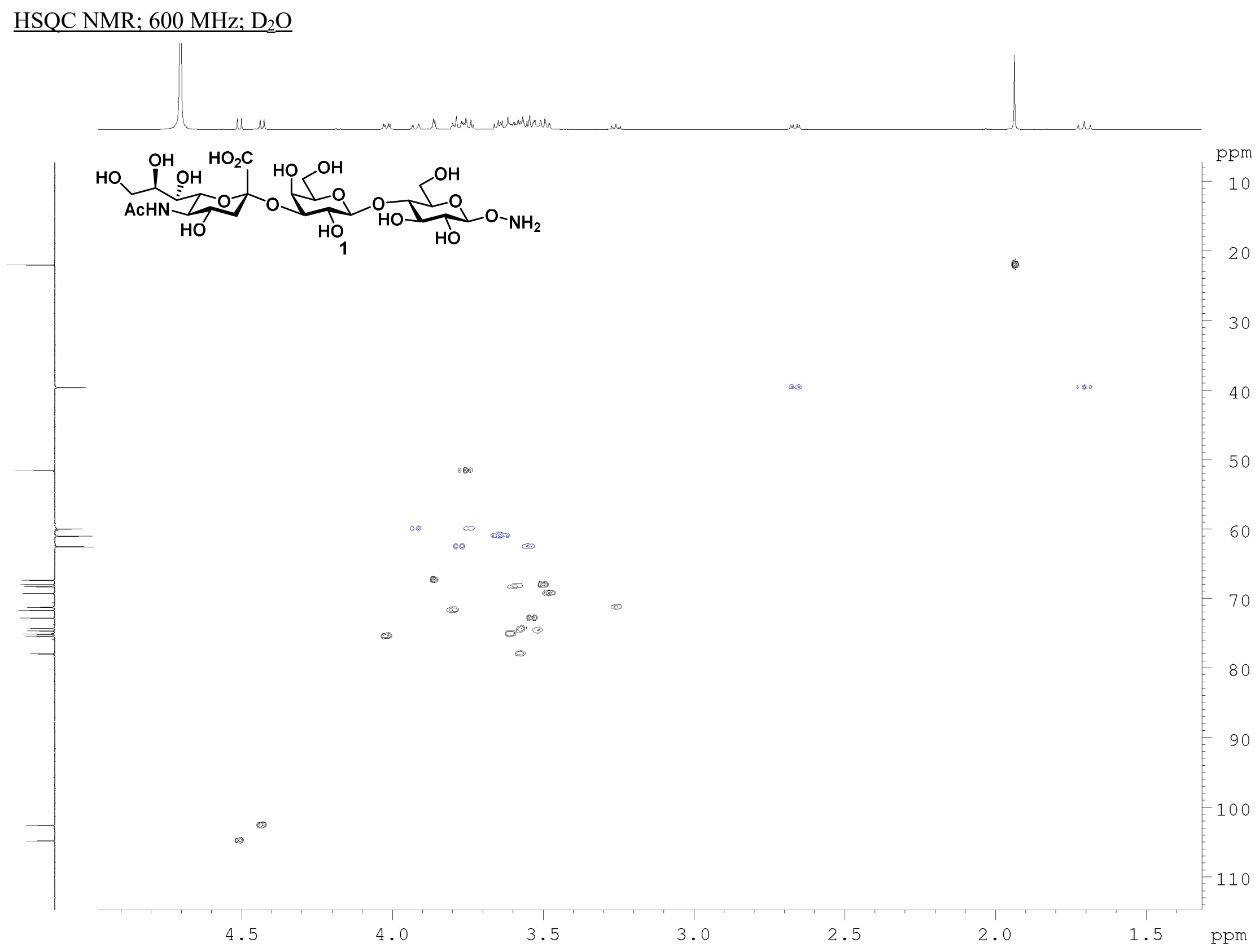




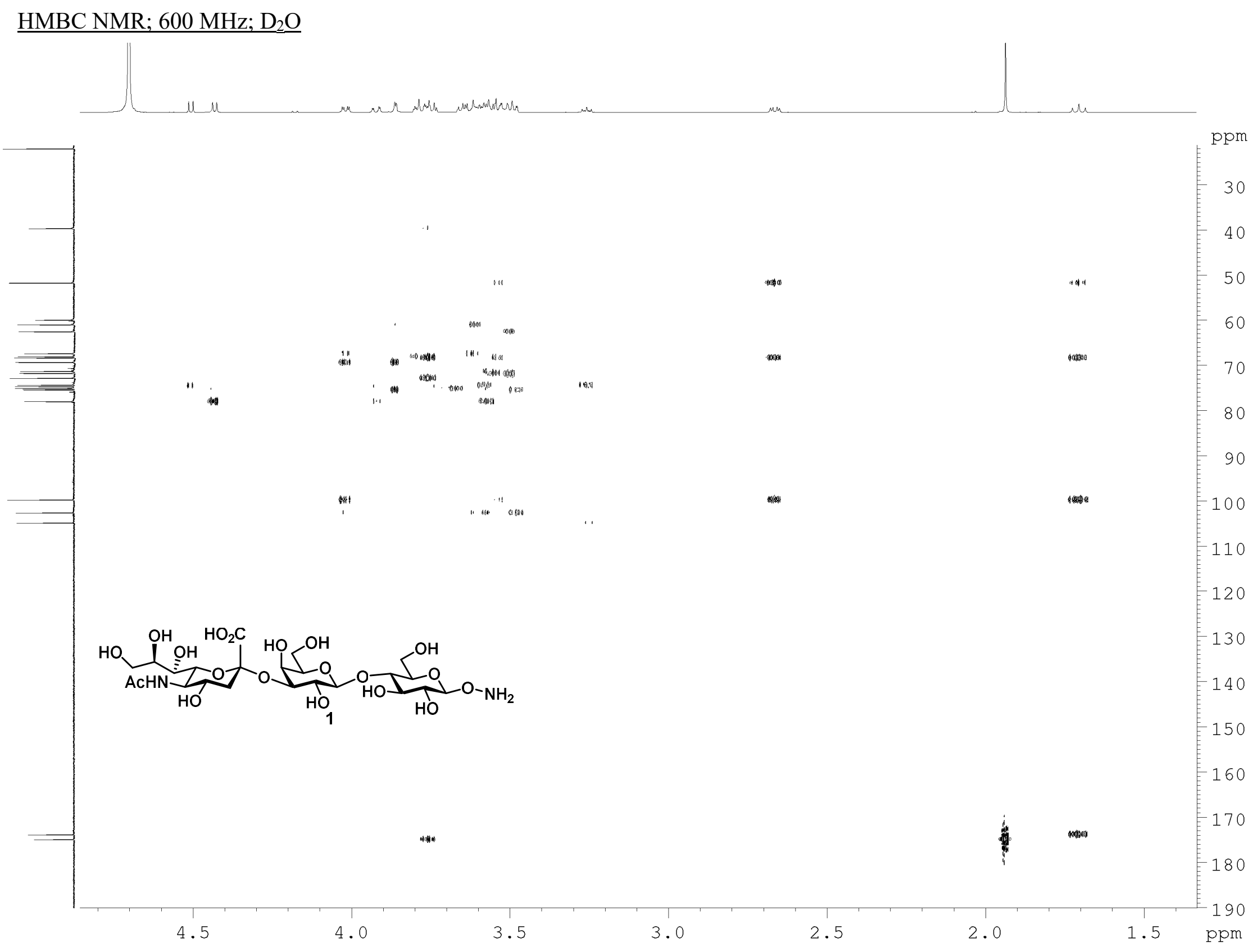




\section{$\underline{\mathrm{HMBC} N M R ; 600 \mathrm{MHz} ; \mathrm{D}_{2} \mathrm{O}}$}

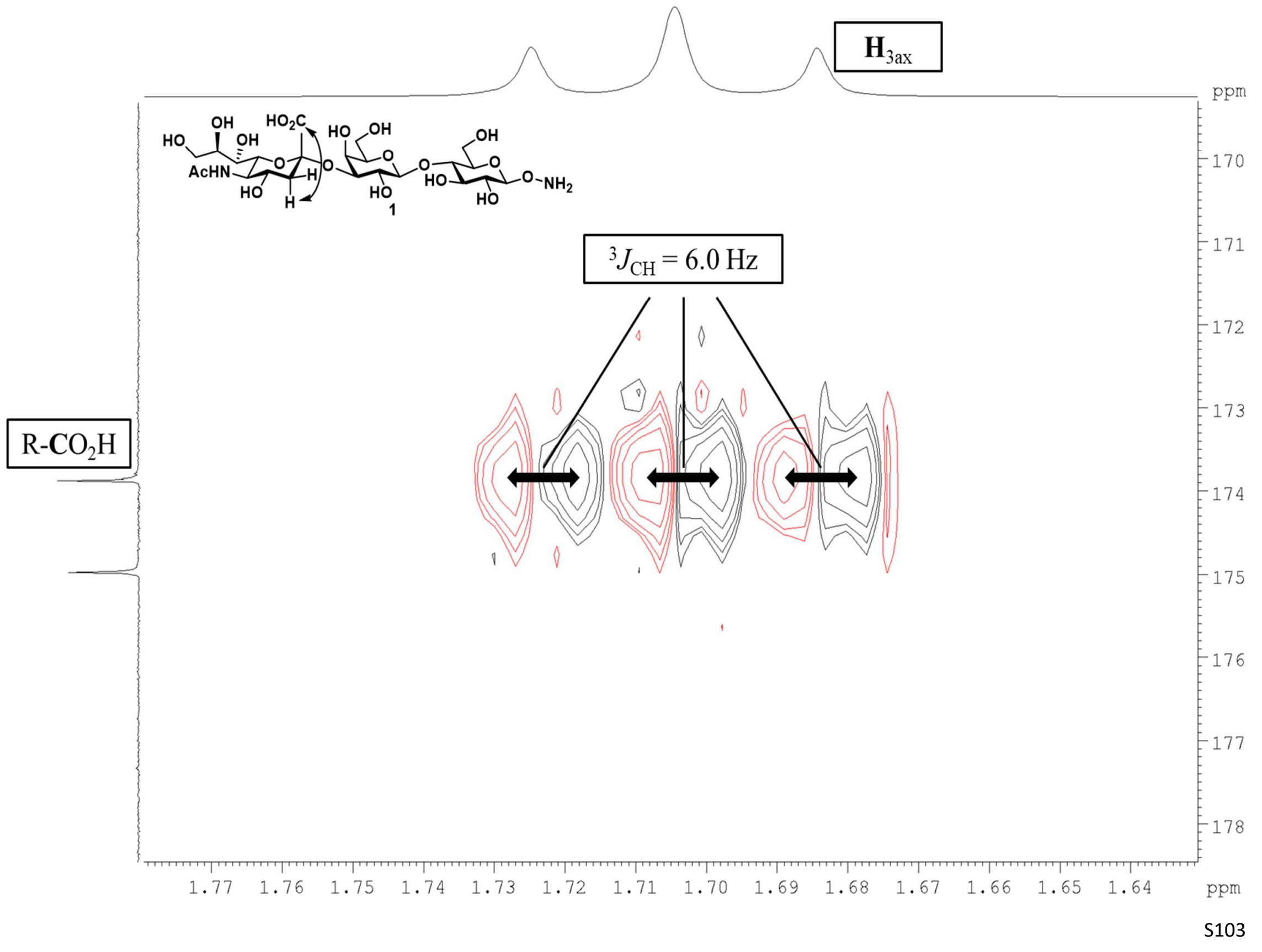




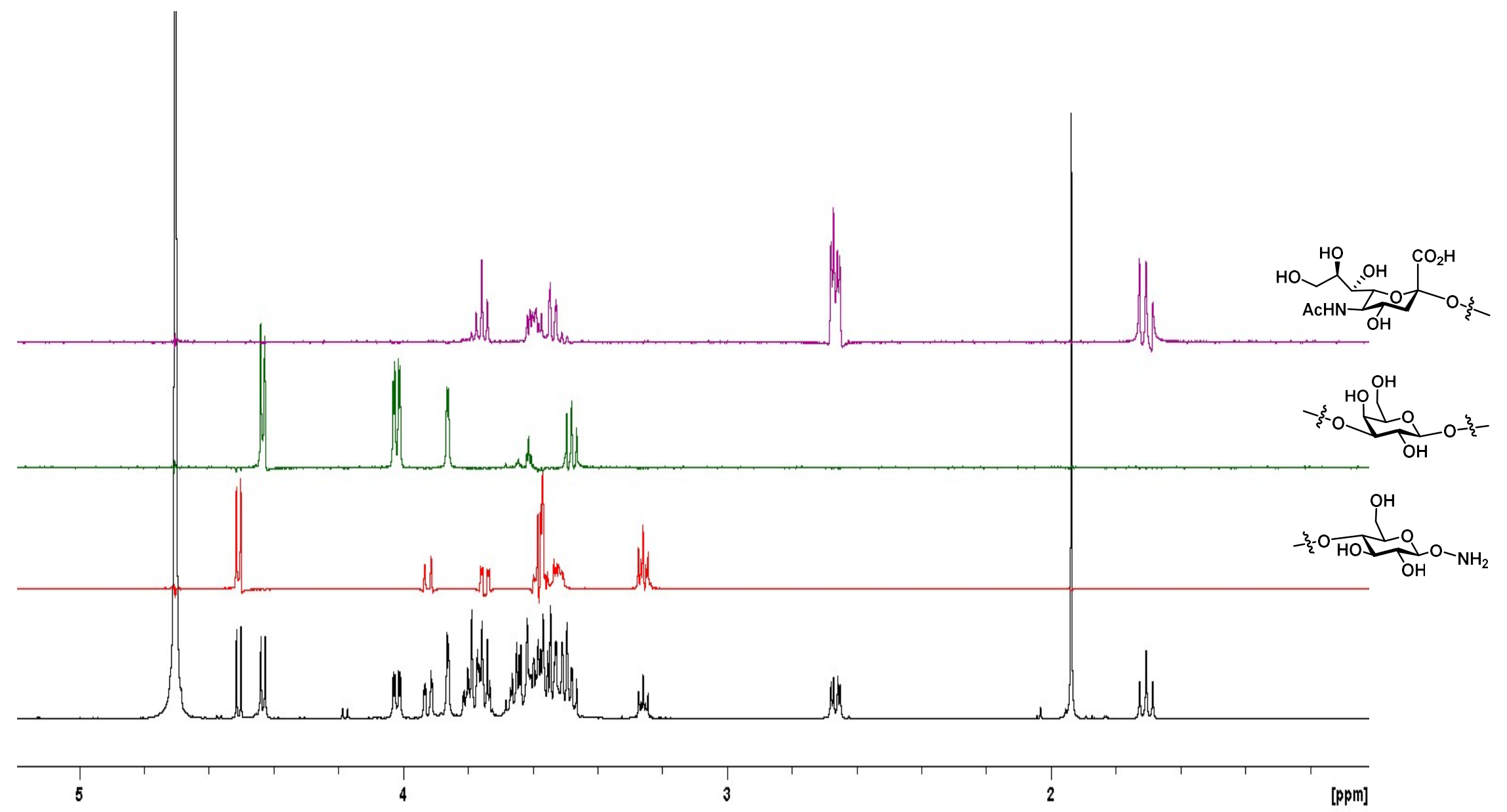


$\underline{{ }^{1} \mathrm{H} \text { NMR; } 600 \mathrm{MHz} ; \mathrm{D}_{2} \mathrm{O} ; 60{ }^{\circ} \mathrm{C} ; \mathrm{D} 1=3.0 \text { seconds }}$

형

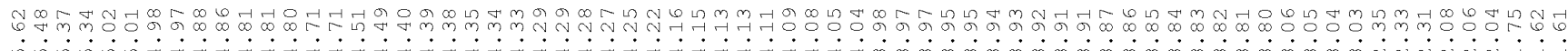
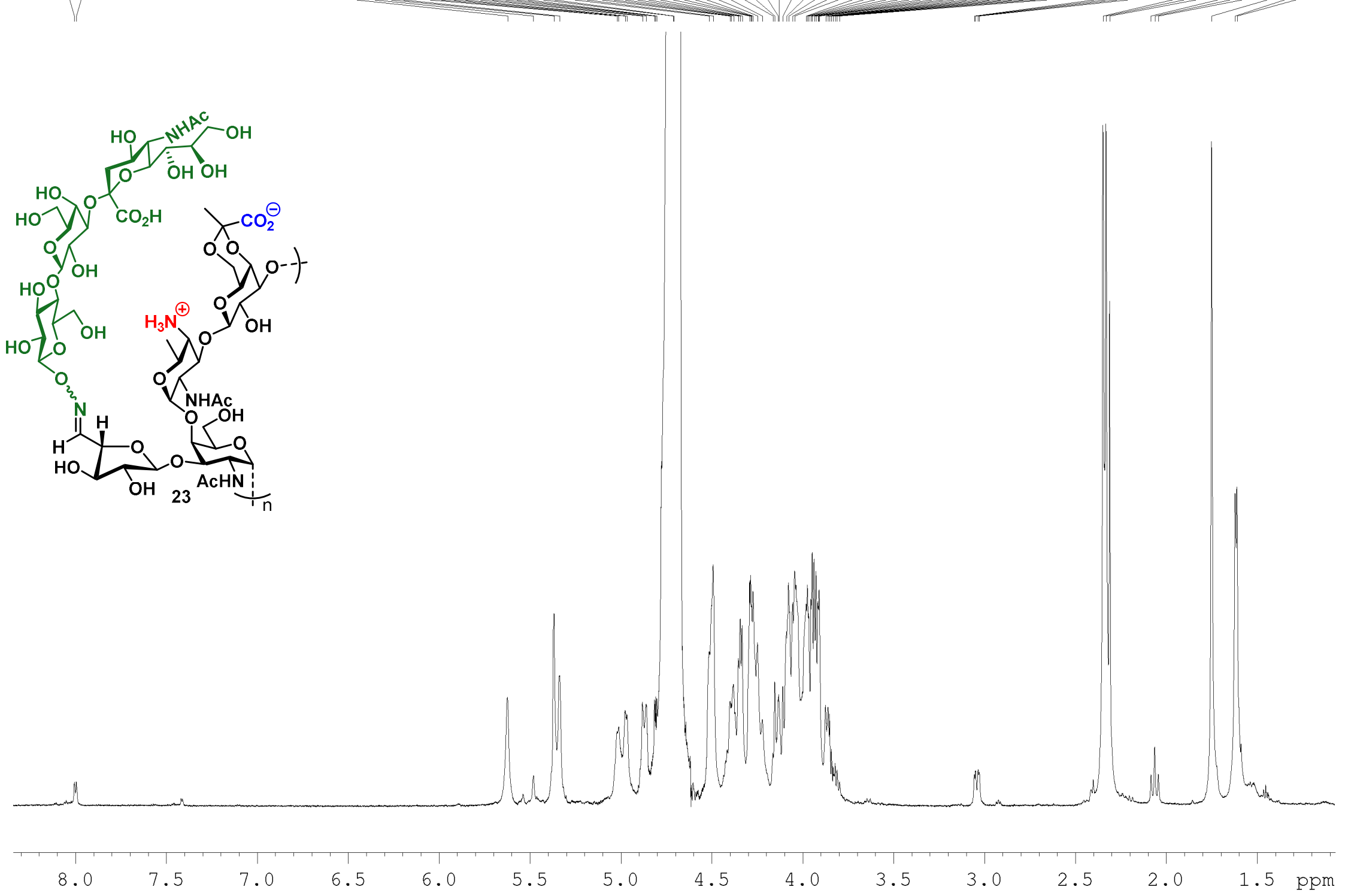
$\underline{{ }^{1} \mathrm{H} \text { NMR; } 600 \mathrm{MHz} ; \mathrm{D}_{2} \mathrm{O} ; 60{ }^{\circ} \mathrm{C} ; \mathrm{D} 1=3.0 \text { seconds }}$

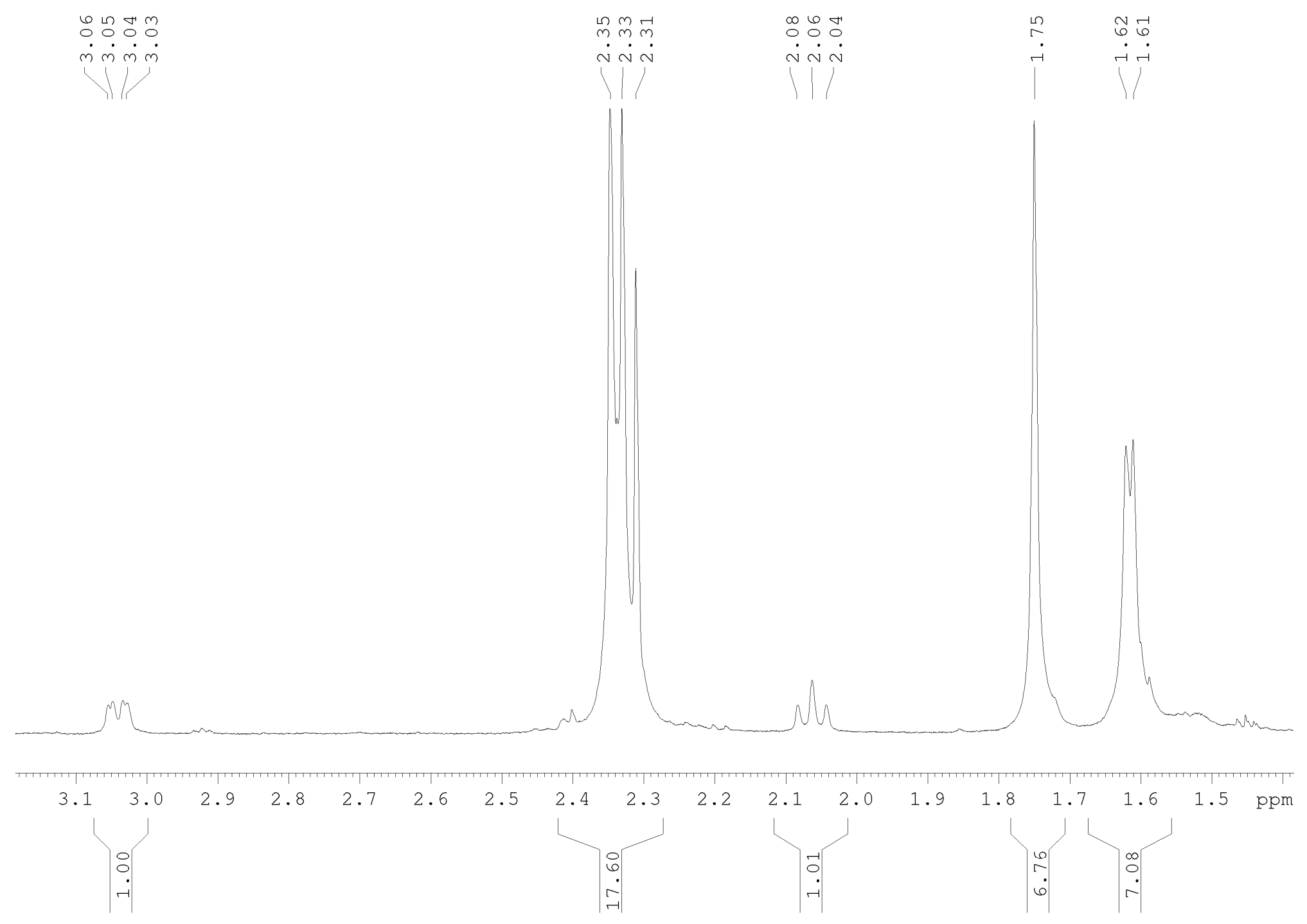


COSY NMR; $600 \mathrm{MHz} ; \mathrm{D}_{2} \mathrm{O} ; 60{ }^{\circ} \mathrm{C}$

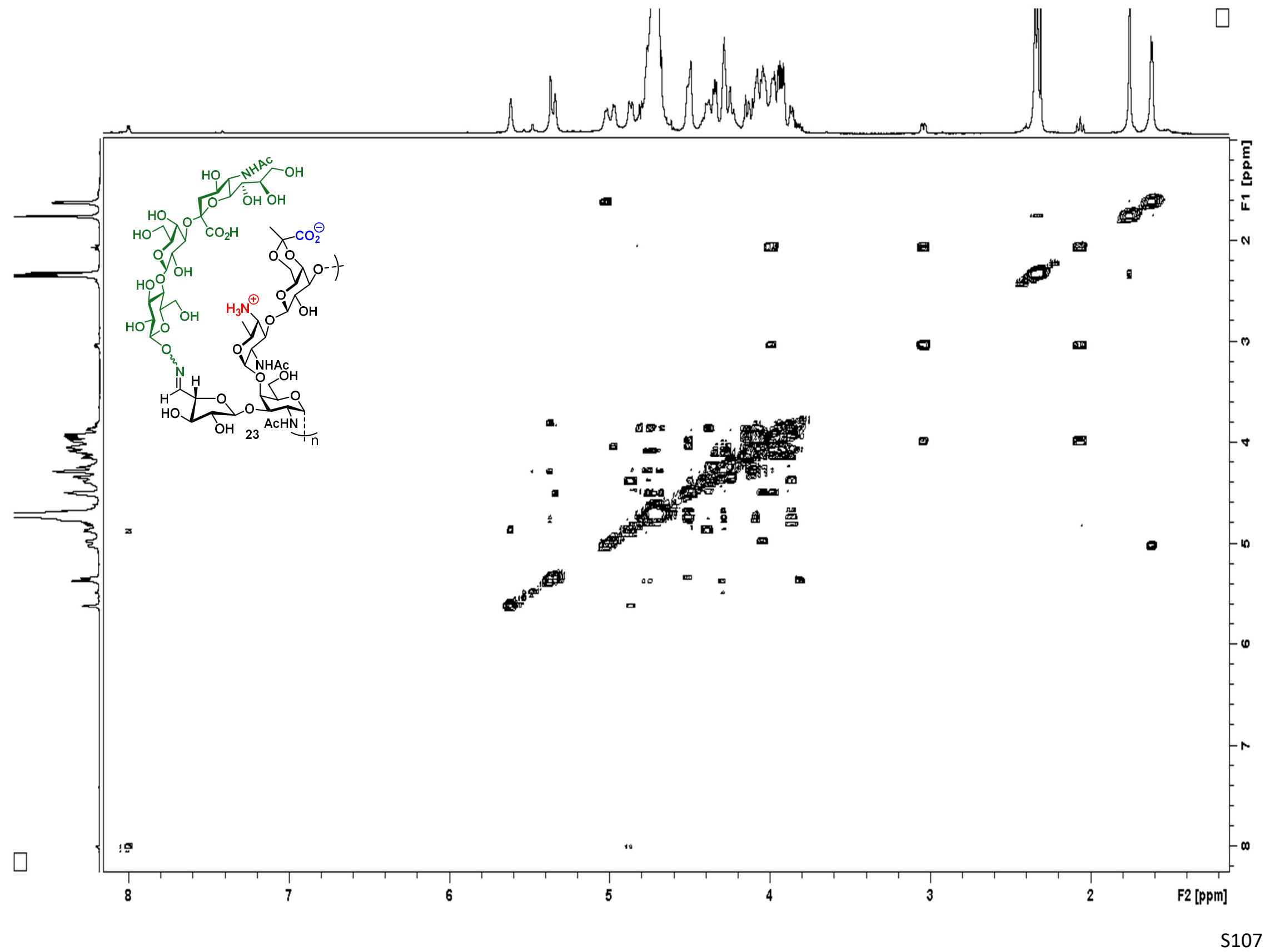




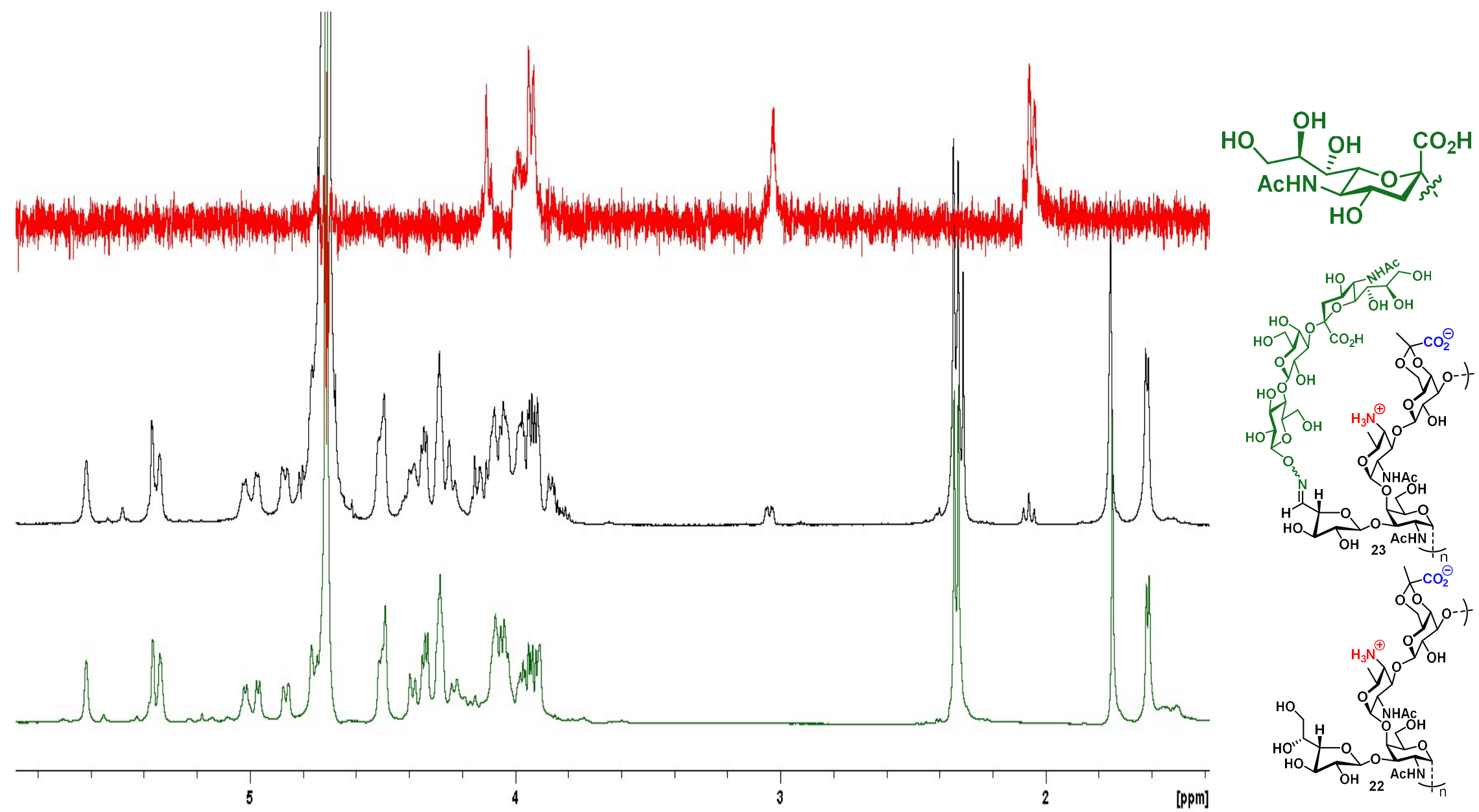




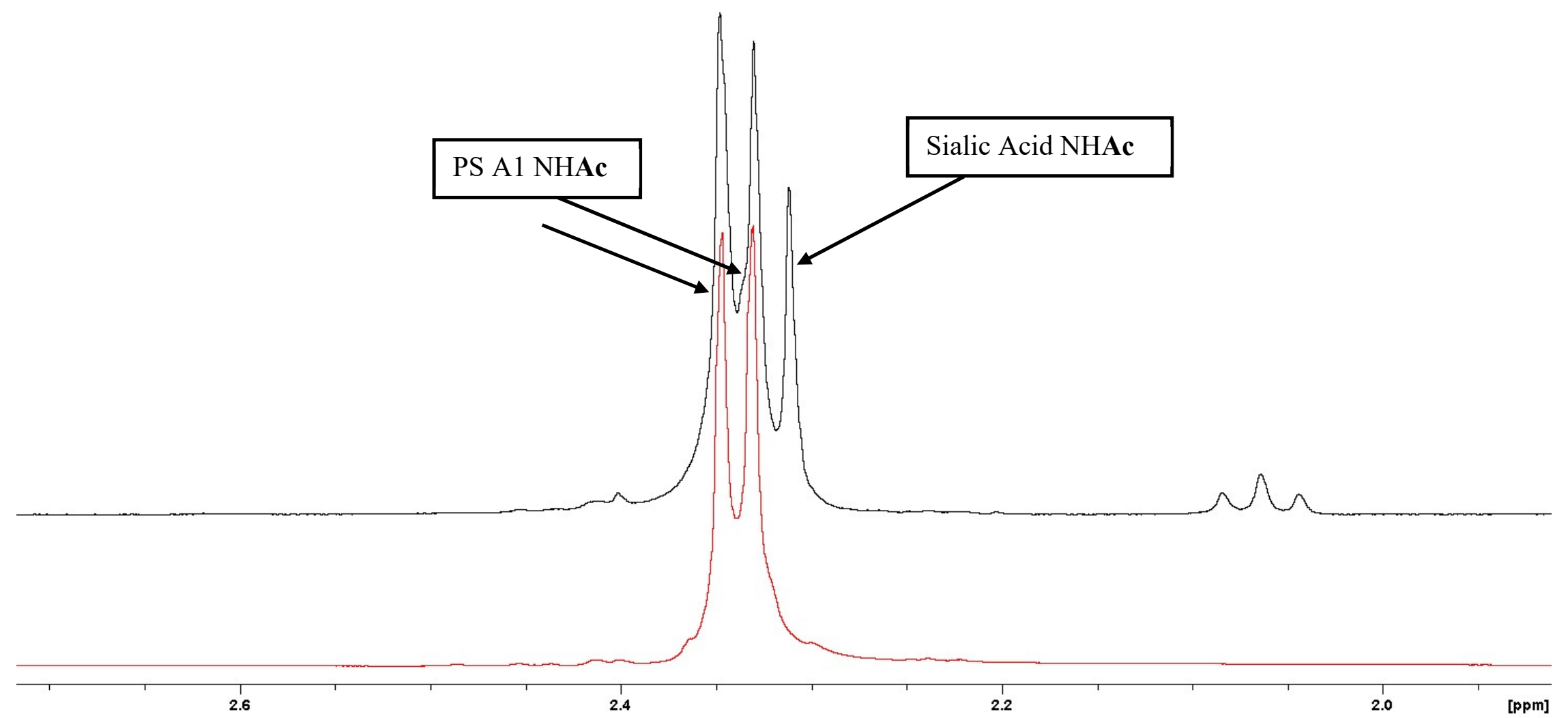

